Geom. Funct. Anal. Vol. 19 (2009) 678-721

DOI 10.1007/s00039-009-0020-8

Published online October 23, 2009

(C) 2009 Birkhäuser Verlag Basel/Switzerland

GAFA Geometric And Functional Analysis

\title{
TIGHT HOMOMORPHISMS AND HERMITIAN SYMMETRIC SPACES
}

\author{
Marc Burger, Alessandra Iozzi And Anna Wienhard
}

\begin{abstract}
We introduce the notion of tight homomorphism into a locally compact group with nonvanishing bounded cohomology and study these homomorphisms in detail when the target is a Lie group of Hermitian type. Tight homomorphisms between Lie groups of Hermitian type give rise to tight totally geodesic maps of Hermitian symmetric spaces. We show that tight maps behave in a functorial way with respect to the Shilov boundary and use this to prove a general structure theorem for tight homomorphisms. Furthermore, we classify all tight embeddings of the Poincaré disk.
\end{abstract}

\section{Contents}

2 Tight Homomorphisms $\quad 682$

2.1 Continuous bounded cohomology _. . . . . . . . . . . . . . . 682

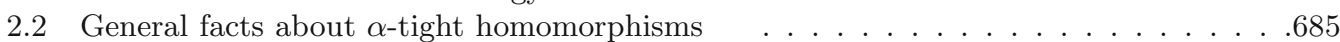

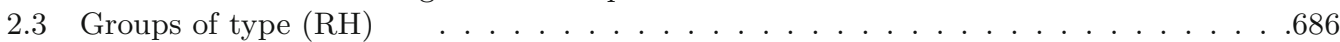

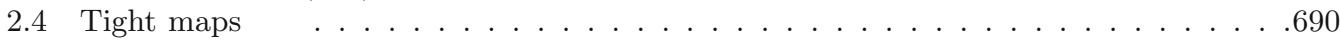

3 Kähler Classes and the Shilov Boundary 692

3.1 Shilov boundary . . . . . . . . . . . . . . . . . . . . . 695

3.2 The Bergmann cocycle and maximal triples _ . . . . . . . . . . . . . 697

3.3 On subdomains of maximal rank _ . . . . . . . . . . . . . . . . . . . . . 699

4 Structure Theorem for Tight Embeddings, I $\quad \mathbf{7 0 0}$

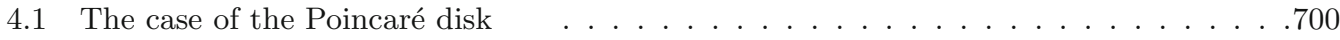

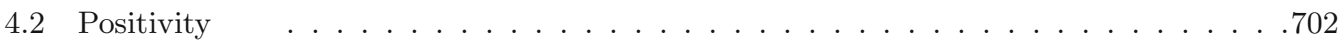

5 Tight Embeddings and Tube-Type Domains $\quad 705$

5.1 The Shilov boundary and tube type domains $\ldots \ldots \ldots \ldots \ldots$. . . . . . . . . . .

5.2 Structure theorem for tight embeddings,II $\ldots \ldots \ldots \ldots \ldots \ldots \ldots$

6 Extensions to Groups of Type (RH) $\quad 710$

Keywords and phrases: Hermitian symmetric spaces, bounded cohomology, Shilov boundary, totally geodesic embedding, tight homomorphism

2000 Mathematics Subject Classification: 53C24, 53C35, 32M15

A.I. and A.W. were partially supported by FNS grant PP002-102765. A.W. was partially supported by the National Science Foundation under agreement No. DMS-0111298 and No. DMS0604665 . 
7 Structure Theorem for Tight Homomorphisms

8 A Criterion for Tightness $\quad \mathbf{7 1 2}$

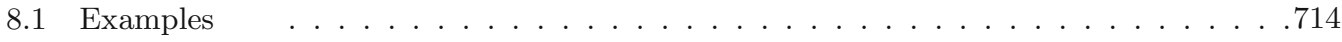

9 Classification of Tight Embeddings of the Poincaré Disk $\quad 716$

$\begin{array}{ll}\text { A Appendix } & \mathbf{7 1 8}\end{array}$

A.1 The norm of the bounded Kähler class _ . . . . . . . . . . . . . . . . . . . . . .718

A.2 Surjection onto lattices . . . . . . . . . . . . . . . . . . . . . . . . . . 719

\section{Introduction}

Let $L, G$ be locally compact second countable topological groups. A continuous homomorphism $\rho: L \rightarrow G$ induces canonical pullback maps $\rho^{*}$ in continuous cohomology and $\rho_{\mathrm{b}}^{*}$ in continuous bounded cohomology. A special feature of continuous bounded cohomology is that it comes equipped with a canonical seminorm $\|\cdot\|$ with respect to which $\rho_{\mathrm{b}}^{*}$ is norm decreasing, that is

$$
\left\|\rho_{\mathrm{b}}^{*}(\alpha)\right\| \leq\|\alpha\| \text { for all } \alpha \in \mathrm{H}_{\mathrm{cb}}^{\bullet}(G, \mathbb{R}) \text {. }
$$

Given a class $\alpha \in \mathrm{H}_{\mathrm{cb}}^{\bullet}(G, \mathbb{R})$ we say that a homomorphism $\rho: L \rightarrow G$ is $\alpha$-tight if the pullback $\rho_{\mathrm{b}}^{*}$ preserves the norm of $\alpha$, that is $\left\|\rho_{\mathrm{b}}^{*}(\alpha)\right\|=\|\alpha\|$.

For the main part of the article we specialize to the situation when the target group $G$ is of Hermitian type, i.e. $G$ is a connected semisimple Lie group with finite center and without compact factors such that its associated symmetric space $\mathcal{X}$ is Hermitian symmetric. Let $\mathcal{J}$ be the $G$-invariant complex structure on $\mathcal{X}$; combining it with the unique $G$-invariant Riemannian metric of minimal holomorphic sectional curvature -1 , gives rise to the Kähler form $\omega_{G} \in \Omega^{2}(\mathcal{X})^{G}$. We denote by $\kappa_{G}^{\mathrm{b}} \in \mathrm{H}_{\mathrm{cb}}^{2}(G, \mathbb{R})$ the bounded continuous cohomology class obtained in the familiar way (see section 2.3) by integration of $\omega_{G}$ over triangles with geodesic sides.

DEFINITION 1. Let $L$ be a locally compact second countable topological group and $G$ a group of Hermitian type. A continuous homomorphism $\rho: L \rightarrow G$ is said to be tight if $\left\|\rho_{\mathrm{b}}^{*}\left(\kappa_{G}^{\mathrm{b}}\right)\right\|=\left\|\kappa_{G}^{\mathrm{b}}\right\|$.

It is implicit in the definition of a tight homomorphism that it depends on the $G$ invariant complex structure $\mathcal{J}$ which is part of the data of the Hermitian symmetric space $\mathcal{X}$.

Fundamental Example. Let $\Gamma<\mathrm{SU}(n, 1)=: G$ be a cocompact lattice and $M:=\Gamma \backslash \mathcal{X}$ the corresponding compact hyperbolic manifold. The (ordinary) Kähler class $\rho^{*}\left(\kappa_{G}\right)$ of a representation $\rho: \Gamma \rightarrow G$, seen as a de Rham class on $M$, can be paired with the Kähler form $\omega_{M}$ on $M$ to give a characteristic number

$$
i_{\rho}:=\frac{\left\langle\rho^{*}\left(\kappa_{G}\right), \omega_{M}\right\rangle}{\left\langle\omega_{M}, \omega_{M}\right\rangle}
$$

which satisfies a Milnor-Wood inequality [BuIo2]

$$
\left|i_{\rho}\right| \leq \mathrm{r}_{\mathcal{X}}
$$


Representations such that $i_{\rho}=\mathrm{r}_{\mathcal{X}}$ are called maximal. (A modification of the above construction leads to the definition of an analogous invariant even in the case of nonuniform lattices, [BuIo2, §5].) Maximal representations are tight [BuIo2, Lem.5.3], and in fact, they are the most important examples of such.

The study of the structure of tight homomorphisms is paramount in the classification of maximal representations of compact surface groups [BuIW1,2], [BuIoLW]. It should be remarked, however, that the scope of the notion goes well beyond this, as for example every surjection of a finitely generated group onto a lattice in $G$ is tight (see Corollary A.4). In particular we have

Proposition 2. Let $\mathbf{M o d}_{g}$ be the mapping class group of a closed surface of genus $g \geq 1$. Then the natural homomorphism $\operatorname{Mod}_{g} \rightarrow \operatorname{Sp}(2 g, \mathbb{R})$ is tight.

One of the main points of this paper is the following structure theorem for tight homomorphisms.

Theorem 3. Let $L$ be a locally compact second countable group, G a connected algebraic group defined over $\mathbb{R}$ such that $G:=\mathbf{G}(\mathbb{R})^{\circ}$ is of Hermitian type. Suppose that $\rho: L \rightarrow G$ is a continuous tight homomorphism. Then,

(1) The Zariski closure $\mathbf{H}:=\overline{\rho(L)}^{Z}$ is reductive.

(2) The centralizer $\mathcal{Z}_{G}(H)$ of $H:=\overline{\rho(L)}^{Z}(\mathbb{R})^{\circ}$ is compact.

(3) The symmetric space $\mathcal{Y}$ corresponding to $H$ is Hermitian and $\mathcal{Y}$ admits a unique $H$-invariant complex structure such that the inclusion $H \rightarrow G$ is tight and positive.

To explain the notion of a positive homomorphism, let us recall that the complex structure $\mathcal{J}$ defines a cone $\mathrm{H}_{\mathrm{c}}^{2}(G, \mathbb{R})^{\geq 0}$ of positive Kähler classes and, via the isomorphism

$$
\mathrm{H}_{\mathrm{cb}}^{2}(G, \mathbb{R}) \stackrel{\cong}{\longrightarrow} \mathrm{H}_{\mathrm{c}}^{2}(G, \mathbb{R})
$$

a cone of bounded positive Kähler classes containing in particular $\kappa_{G}^{\mathrm{b}}$. A continuous homomorphism $\rho: G_{1} \rightarrow G_{2}$ between two groups of Hermitian type is said to be positive if $\rho_{\mathrm{b}}^{*} \kappa_{G_{2}}^{\mathrm{b}} \in \mathrm{H}_{\mathrm{cb}}^{2}\left(G_{1}, \mathbb{R}\right) \geq 0$.

As an immediate application of Theorem 3, we have

Corollary 4. Let $\mathbf{G}$ be a connected algebraic group defined over $\mathbb{R}$ such that $G:=\mathbf{G}(\mathbb{R})^{\circ}$ is of Hermitian type and let $\rho: \Gamma \rightarrow G$ be a maximal representation of a lattice $\Gamma<\mathrm{SU}(n, 1)$. Then,

(1) The Zariski closure $\mathbf{H}:=\overline{\rho(\Gamma)}^{Z}$ is reductive.

(2) The centralizer $\mathcal{Z}_{G}(H)$ of $H:=\overline{\rho(\Gamma)}^{Z}(\mathbb{R})^{\circ}$ is compact.

(3) The symmetric space $\mathcal{Y}$ corresponding to $H$ is Hermitian and $\mathcal{Y}$ admits a unique $H$-invariant complex structure such that the inclusion $H \rightarrow G$ is tight and positive.

In the case in which $\Gamma$ is the fundamental group of an oriented compact surface possibly with boundary, one can reach much stronger conclusions, as for example faithfulness and discreteness of $\rho,[$ BuIW1,2], [BuIoLW]. 
Our study of tight homomorphisms relies on the study of a parallel notion of tightness for totally geodesic maps between symmetric spaces. Namely, let $\mathcal{X}_{1}, \mathcal{X}_{2}$ be Hermitian symmetric spaces of noncompact type.

Definition 5. A totally geodesic map $f: \mathcal{X}_{1} \rightarrow \mathcal{X}_{2}$ is said to be tight if

$$
\sup _{\Delta \subset \mathcal{X}_{1}} \int_{\Delta} f^{*} \omega_{G_{2}}=\sup _{\Delta \subset \mathcal{X}_{2}} \int_{\Delta} \omega_{G_{2}}
$$

where the supremum is taken over all smooth oriented triangles $\Delta$ with geodesic sides in $\mathcal{X}_{1}$ and $\mathcal{X}_{2}$, respectively.

A first immediate fact is the following:

Proposition 6. Let $G_{1}, G_{2}$ be Lie groups of Hermitian type and let $\mathcal{X}_{1}, \mathcal{X}_{2}$ be the corresponding symmetric spaces. A homomorphism $\rho: G_{1} \rightarrow G_{2}$ is tight if and only if the corresponding totally geodesic map $f: \mathcal{X}_{1} \rightarrow \mathcal{X}_{2}$ is tight.

Tight embeddings behave nicely at infinity. Recall that the Shilov boundary $\check{S}_{\mathcal{X}}$ of the Hermitian symmetric space $\mathcal{X}$ is the unique closed $G$-orbit in the (topological) compactification of the bounded symmetric domain realization $\mathcal{D}$ of $\mathcal{X}$, and can be identified with $G / Q$, where $Q<G$ is an appropriate parabolic subgroup. Two points $x, y$ in $\check{S}_{\mathcal{X}}$ are said to be transverse if $(x, y)$ is in the unique open $G$-orbit in $\check{S}_{\mathcal{X}} \times \check{S}_{\mathcal{X}}$.

Theorem 7. Let $G_{1}, G_{2}$ be Lie groups of Hermitian type and $\check{S}_{1}, \check{S}_{2}$ the Shilov boundaries of the associated symmetric spaces. Let $\rho: G_{1} \rightarrow G_{2}$ be a continuous tight homomorphism and $f: \mathcal{X}_{1} \rightarrow \mathcal{X}_{2}$ the corresponding totally geodesic tight map. Then there exists a $\rho$-equivariant continuous map $\check{f}: \breve{S}_{1} \rightarrow \check{S}_{2}$ which extends $f$ and which maps transverse pairs to transverse pairs.

Using this theorem we can establish a general existence result for boundary maps. Let $\Gamma$ be a countable discrete group and $(B, \nu)$ a Poisson boundary for $\Gamma$. Recall that under these conditions, the amenability of the $\Gamma$-action on $(B, \nu)$ insures the existence of a $\rho$-equivariant measurable map from $B$ to the space of probability measures on $G / P$, where $P$ is a minimal parabolic in $G$. Under some conditions, such as for instance Zariski density of the image of the representation $\rho$, one can deduce the existence of such a map with values in $G / P$ (see [BuIo1], [BuIoW3]).

For tight homomorphisms we have the general existence result:

Theorem 8. Let $\mathbf{G}$ be a semisimple algebraic group defined over $\mathbb{R}$ such that $G:=\mathbf{G}(\mathbb{R})$ is of Hermitian type and let $\rho: \Gamma \rightarrow G$ be a tight homomorphism. Then there exists a $\rho$-equivariant measurable $\operatorname{map} \varphi: B \rightarrow \check{S}_{\mathcal{X}}$.

Recall that Hermitian symmetric spaces fall into two classes, according to whether or not they admit a realization generalizing the upper half plane model of the Poincaré disk. Namely, a Hermitian symmetric space is of tube type if it is biholomorphically equivalent to a domain $V \oplus i \Omega$ where $\Omega \subset V$ is a proper open cone in the real vector space $V$. For any Hermitian symmetric space $\mathcal{X}$ maximal subdomains of tube type exist, they are of the same rank as $\mathcal{X}$, holomorphically embedded and pairwise conjugate. 
Theorem 9. Let $\rho: G_{1} \rightarrow G_{2}$ be a tight homomorphism and $f: \mathcal{X}_{1} \rightarrow \mathcal{X}_{2}$ the corresponding tight map. Then,

(1) If $\mathcal{X}_{1}$ is of tube type, then there exists a unique maximal subdomain of tube type $T \subset \mathcal{X}_{2}$ such that $f\left(\mathcal{X}_{1}\right) \subset T$. Moreover $\rho\left(G_{1}\right)$ preserves $T$.

(2) If $\operatorname{ker} \rho$ is finite and $\mathcal{X}_{2}$ is of tube type, then $\mathcal{X}_{1}$ is of tube type.

Our main tool to study tight embeddings and tight homomorphisms is the concept of diagonal disk. Recall that a maximal polydisk in $\mathcal{X}$ is the image of a holomorphic and totally geodesic embedding $t: \mathbb{D}^{\mathrm{r} \mathcal{X}} \rightarrow \mathcal{X}$. Maximal polydisks arise as complexifications of maximal flats in $\mathcal{X}$ and are conjugate. It is easy to check that maximal polydisks are tightly embedded. A diagonal disk in $\mathcal{X}$ is the image of the diagonal $\Delta(\mathbb{D}) \subset \mathbb{D}^{\mathrm{r} \mathcal{X}}$ under the embedding $t: \mathbb{D}^{\mathrm{r} \mathcal{X}} \rightarrow \mathcal{X}$. Diagonal disks are precisely tight and holomorphically embedded disks in $\mathcal{X}$.

Using diagonal disks we can give a simple criterion for tightness of a totally geodesic embedding $f: \mathcal{X}_{1} \rightarrow \mathcal{X}_{2}$ in terms of the corresponding homomorphism of Lie algebras (see Lemma 8.1). We apply this criterion to classify tight embeddings of the Poincaré disk and obtain:

Theorem 10. Suppose that $\mathcal{X}$ is a Hermitian symmetric space and $f: \mathbb{D} \rightarrow \mathcal{X}$ is a tight embedding. Then the smallest Hermitian symmetric subspace $\mathcal{Y} \subset \mathcal{X}$ containing $f(\mathbb{D})$ is a product $\mathcal{Y}=\Pi_{i=1}^{k} \mathcal{Y}_{i}$ of Hermitian symmetric subspaces $\mathcal{Y}_{i}$ of $\mathcal{X}$, where $\mathcal{Y}_{i}$ is the Hermitian symmetric space associated to the symplectic group $\operatorname{Sp}\left(2 n_{i}, \mathbb{R}\right)$. Moreover, $\sum_{i=1}^{k} n_{i} \leq r_{\mathcal{X}}$ and the embedding $f: \mathbb{D} \rightarrow \mathcal{Y}_{i}$ is equivariant with respect to the irreducible representation $\mathrm{SL}(2, \mathbb{R}) \rightarrow \operatorname{Sp}\left(2 n_{i}, \mathbb{R}\right)$.

Tight embeddings are never totally real but they are also not necessarily holomorphic. The irreducible representations $\mathrm{SL}(2, \mathbb{R}) \rightarrow \operatorname{Sp}(2 n, \mathbb{R})$ provide examples of non-holomorphic tight embeddings of the Poincaré disk when $n \geq 2$.

We are not aware of an example of a non-holomorphic tight embedding of an irreducible Hermitian symmetric space of rank $r_{\mathcal{X}} \geq 2$. It might be that all tight homomorphisms of higher-rank Hermitian symmetric spaces are holomorphic.

We suspect that tight embeddings of Hermitian symmetric spaces that are not of tube type are always holomorphic. For tight embeddings of $\mathbb{C} \mathbb{H}^{n}$ into classical Hermitian symmetric spaces of rank 2 this can be deduced from [KoM].

Acknowledgments. We thank Domingo Toledo for useful discussions about tight embeddings of complex hyperbolic spaces.

\section{Tight Homomorphisms}

2.1 Continuous bounded cohomology. In this section we recall some properties of bounded continuous cohomology which are used in the sequel. For proofs and a comprehensive account of continuous bounded cohomology the reader is referred to $[\mathrm{M}],[\mathrm{BuM} 2]$.

If $G$ is a locally compact second countable group, then

$$
\mathrm{C}_{\mathrm{b}}\left(G^{k+1}, \mathbb{R}\right):=\left\{f: G^{k+1} \rightarrow \mathbb{R}: f \text { is continuous and }\|f\|_{\infty}<\infty\right\}
$$


is a $G$-module via the action

$$
(h f)\left(g_{0}, \ldots, g_{k}\right)=f\left(h^{-1} g_{0}, \ldots, h^{-1} g_{k}\right) .
$$

The continuous bounded cohomology $\mathrm{H}_{\mathrm{cb}}^{\bullet}(G, \mathbb{R})$ of $G$ with coefficients in $\mathbb{R}$ is the cohomology of the complex of $G$-invariants

$$
0 \longrightarrow \mathrm{C}_{\mathrm{b}}(G, \mathbb{R})^{G \stackrel{d}{\longrightarrow}} \mathrm{C}_{\mathrm{b}}\left(G^{2}, \mathbb{R}\right)^{G \stackrel{d}{\longrightarrow} \cdots}
$$

where $d$ is the usual homogeneous coboundary operator defined for $f \in \mathrm{C}_{\mathrm{b}}\left(G^{k}, \mathbb{R}\right)$ by

$$
d f\left(g_{0}, \ldots g_{k}\right):=\sum_{i=0}^{k} f\left(g_{0}, \ldots, \hat{g}_{i}, \ldots, g_{k}\right) .
$$

The supremum norm gives $\mathrm{C}_{\mathrm{b}}\left(G^{\bullet+1}, \mathbb{R}\right)^{G}$ the structure of a Banach space and induces a canonical seminorm $\|\cdot\|$ on $\mathrm{H}_{\mathrm{cb}}^{\bullet}(G, \mathbb{R})$

$$
\|\alpha\|=\inf _{[f]=\alpha}\|f\|_{\infty} .
$$

The inclusion of complexes $\mathrm{C}_{\mathrm{b}}\left(G^{\bullet+1}, \mathbb{R}\right) \subset \mathrm{C}\left(G^{\bullet+1}, \mathbb{R}\right)$, where $\mathrm{C}\left(G^{\bullet+1}, \mathbb{R}\right)$ denotes the space of continuous real-valued functions, induces a natural comparison map

$$
\mathrm{c}_{G}: \mathrm{H}_{\mathrm{cb}}^{\bullet}(G, \mathbb{R}) \rightarrow \mathrm{H}_{\mathrm{c}}^{\bullet}(G, \mathbb{R})
$$

from continuous bounded cohomology to continuous cohomology. Moreover, any continuous homomorphism $\rho: L \rightarrow G$ of locally compact groups induces canonical pullbacks both in continuous cohomology and in continuous bounded cohomology, such that the diagram

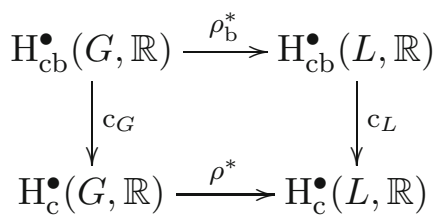

commutes. In particular, if $L<G$ is a closed subgroup, the pullback given by the inclusion is the restriction map.

Proposition 2.1. (1) Let $L$ be a locally compact second countable group and $L_{0}<L$ a closed subgroup. If $L_{0}$ is of finite index in $L$, then the restriction map

$$
\begin{aligned}
\mathrm{H}_{\mathrm{cb}}^{\bullet}(L, \mathbb{R}) & \longrightarrow \mathrm{H}_{\mathrm{cb}}^{\bullet}\left(L_{0}, \mathbb{R}\right) \\
\kappa & \left.\longmapsto \kappa\right|_{L_{0}}
\end{aligned}
$$

is an isometric isomorphism [BuM2, Prop. 2.4.2].

(2) If $R \triangleleft G$ is a closed amenable normal subgroup, the canonical projection $p: G \rightarrow G / R$ induces an isometric isomorphism via the pullback

$$
p_{\mathrm{b}}^{*}: \mathrm{H}_{\mathrm{cb}}^{\bullet}(G / R, \mathbb{R}) \longrightarrow \mathrm{H}_{\mathrm{cb}}^{\bullet}(G, \mathbb{R})
$$

in continuous bounded cohomology, [M, Cor. 8.5.2].

(3) The seminorm $\|\cdot\|$ on $\mathrm{H}_{\mathrm{cb}}^{2}(G, \mathbb{R})$ is a norm which turns it into a Banach space, [BuM2, Th.2]. 
(4) If $G=G_{1} \times \cdots \times G_{n}$ is a direct product of locally compact second countable groups, then the map

$$
\begin{aligned}
\mathrm{H}_{\mathrm{cb}}^{2}(G, \mathbb{R}) & \longrightarrow \prod_{i=1}^{n} \mathrm{H}_{\mathrm{cb}}^{2}\left(G_{i}, \mathbb{R}\right) \\
\kappa & \longmapsto\left(\kappa_{\left.\right|_{G_{i}}}\right)_{i=1}^{n}
\end{aligned}
$$

into the Cartesian product of the continuous bounded cohomology of the factors, is an isometric isomorphism, [BuM2, Cor.4.4.1], that is

$$
\|\kappa\|=\sum_{i=1}^{n}\left\|\kappa_{\left.\right|_{G_{i}}}\right\| .
$$

By a slight abuse of notation, we indicate by $\left.\kappa\right|_{G_{i}}$ the pullback of $\kappa \in \mathrm{H}_{\mathrm{cb}}^{2}(G, \mathbb{R})$ by the homomorphism

$$
\begin{aligned}
G_{i} & \longrightarrow G=G_{1} \times \cdots \times G_{n} \\
g_{i} \longmapsto & \left(e, \ldots, g_{i}, \ldots, e\right) .
\end{aligned}
$$

REMARK 2.2. (1) The statement in Proposition 2.1(1) is true more generally if there exists an $L$-invariant mean on $L / L_{0}$, but we shall not need this here.

(2) The fact that the isomorphism $\mathrm{H}_{\mathrm{cb}}^{2}(G, \mathbb{R}) \cong \prod_{i=1}^{n} \mathrm{H}_{\mathrm{cb}}^{2}\left(G_{i}, \mathbb{R}\right)$ is isometric is not stated as such in [BuM2], but it follows from the proof. Moreover an explicit inverse to the map $\mathrm{H}_{\mathrm{cb}}^{2}(G, \mathbb{R}) \rightarrow \prod_{i=1}^{n} \mathrm{H}_{\mathrm{cb}}^{2}\left(G_{i}, \mathbb{R}\right)$ in $(2.2)$ is given by

$$
\begin{aligned}
\prod_{i=1}^{n} \mathrm{H}_{\mathrm{cb}}^{2}\left(G_{i}, \mathbb{R}\right) & \longrightarrow \mathrm{H}_{\mathrm{cb}}^{2}(G, \mathbb{R}) \\
\left(\kappa_{i}\right)_{i=1}^{n} & \longmapsto \sum_{i=1}^{n}\left(p_{i}\right)_{\mathrm{b}}^{*} \kappa_{i},
\end{aligned}
$$

where $p_{i}: G \rightarrow G_{i}$ is the projection onto the $i$-th factor.

We record the following fact as a consequence of Proposition 2.1.

Corollary 2.3. Let $L=H \cdot R$, where $L$ is a locally compact second countable group, $H$ and $R$ are closed subgroups of $L$. We assume that $R$ is amenable and normal in $L$. Then the restriction map

$$
\mathrm{H}_{\mathrm{cb}}^{\bullet}(L, \mathbb{R}) \longrightarrow \mathrm{H}_{\mathrm{cb}}^{\bullet}(H, \mathbb{R})
$$

is an isometric isomorphism.

Proof. We have the following commutative diagram

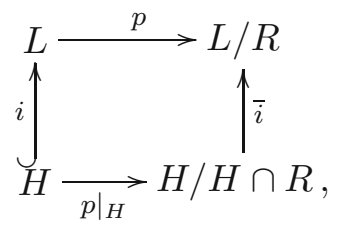

where $\bar{i}$ is the topological isomorphism induced by $i$; according to Proposition 2.1(2), $p_{\mathrm{b}}^{*}$ as well as $\left(\left.p\right|_{H}\right)_{\mathrm{b}}^{*}$ and $\bar{i}_{\mathrm{b}}^{*}$ induce isometric isomorphisms. This implies the assertion about $i_{\mathrm{b}}^{*}$. 
2.2 General facts about $\boldsymbol{\alpha}$-tight homomorphisms. The pullback $\rho_{\mathrm{b}}^{*}$ is seminorm decreasing with respect to the canonical seminorm in continuous bounded cohomology, that is $\left\|\rho_{\mathrm{b}}^{*}(\alpha)\right\| \leq\|\alpha\|$ for all $k \in \mathbb{N}$ and all $\alpha \in \mathrm{H}_{\mathrm{cb}}^{k}(G, \mathbb{R})$. So, it is natural to give the following

DeFinition 2.4. Let $L, G$ be locally compact second countable topological groups and $\alpha \in \mathrm{H}_{\mathrm{cb}}^{\bullet}(G, \mathbb{R})$ a continuous bounded cohomology class. A continuous homomorphism $\rho: L \rightarrow G$ is said to be $\alpha$-tight if $\left\|\rho_{\mathrm{b}}^{*}(\alpha)\right\|=\|\alpha\|$.

Lemma 2.5. Let $L, G, H$ be locally compact second countable groups. Suppose that $\rho: L \rightarrow H, \psi: H \rightarrow G$ are continuous homomorphisms. Let $\alpha \in \mathrm{H}_{\mathrm{cb}}^{\bullet}(G, \mathbb{R})$. Then $\psi$ is $\alpha$-tight and $\rho$ is $\psi_{\mathrm{b}}^{*}(\alpha)$-tight if and only if $\psi \circ \rho$ is $\alpha$-tight.

Proof. The statement follows immediately from the chain of inequalities

$$
\left\|(\psi \circ \rho)_{\mathrm{b}}^{*} \alpha\right\|=\left\|\rho_{\mathrm{b}}^{*} \psi_{\mathrm{b}}^{*} \alpha\right\| \leq\left\|\psi_{\mathrm{b}}^{*} \alpha\right\| \leq\|\alpha\| \text {. }
$$

The following properties of tight homomorphisms are straightforward consequences of the properties of continuous bounded cohomology summarized in Proposition 2.1.

Lemma 2.6. Let $L, G$ be locally compact second countable topological groups, $\alpha \in \mathrm{H}_{\mathrm{cb}}^{\bullet}(G, \mathbb{R})$ and $\rho: L \rightarrow G$ an $\alpha$-tight homomorphism.

(1) Let $H<G$ be a closed subgroup. If the image $\rho(L)$ is contained in $H$ then $\rho$ is $\alpha_{\left.\right|_{H}}$-tight and $\left\|\alpha_{\left.\right|_{H}}\right\|=\|\alpha\|$.

(2) Let $L_{0}<L$ be a closed subgroup of finite index in $L$. Then $\rho_{\left.\right|_{L_{0}}}$ is $\alpha$-tight and

$$
\left\|\left(\rho_{L_{0}}\right)_{\mathrm{b}}^{*} \alpha\right\|=\left\|\rho_{\mathrm{b}}^{*} \alpha\right\|=\|\alpha\| .
$$

(3) Let $R \triangleleft G$ be a closed amenable normal subgroup, $p: G \rightarrow G / R$ the canonical projection. Then the homomorphism $p \circ \rho: L \rightarrow G / R$ is tight with respect to the class $\left(p_{\mathrm{b}}^{*}\right)^{-1}(\alpha) \in \mathrm{H}_{\mathrm{cb}}^{2}(G / R, \mathbb{R})$.

(4) Let $\alpha \in \mathrm{H}_{\mathrm{cb}}^{2}(G, \mathbb{R})$ and if $G:=G_{1} \times \cdots \times G_{n}$, let $p_{i}: G \rightarrow G_{i}$ be the projection onto the $i$-th factor, $i=1, \ldots, n$. Then $\rho_{i}=p_{i} \circ \rho: L \rightarrow G_{i}$ is $\alpha_{\left.\right|_{G_{i}}}$-tight for all $i$.

Proof. (1) Since $\rho(L)$ is contained in $H$ we have that $\rho_{\mathrm{b}}^{*} \alpha=\rho_{\mathrm{b}}^{*}\left(\alpha_{\left.\right|_{H}}\right)$. If $\rho$ is $\alpha$-tight, then

$$
\|\alpha\|=\left\|\rho_{\mathrm{b}}^{*} \alpha\right\|=\left\|\rho_{\mathrm{b}}^{*}\left(\alpha_{\left.\right|_{H}}\right)\right\| \leq\left\|\alpha_{\left.\right|_{H}}\right\|
$$

Since $H<G$ is a subgroup we have that $\left\|\alpha_{\left.\right|_{H}}\right\| \leq\|\alpha\|$ and the claim follows.

(2) Since $\left(\rho_{L_{0}}\right)_{\mathrm{b}}^{*} \alpha$ is the restriction to $L_{0}$ of the class $\left(\rho_{\mathrm{b}}^{*} \alpha\right) \in \mathrm{H}_{\mathrm{cb}}^{\bullet}(L, \mathbb{R})$, by Proposition 2.1(1) and tightness of $\rho$ we have that

$$
\left\|\left(\rho_{L_{0}}\right)_{\mathrm{b}}^{*} \alpha\right\|=\left\|\rho_{\mathrm{b}}^{*} \alpha\right\|=\|\alpha\| .
$$

(3) The facts that $p_{\mathrm{b}}^{*}$ is an isometric isomorphism (Proposition 2.1(2)) and that $\rho$ is $\alpha$-tight give rise to the following chain of equalities:

$$
\left\|(p \circ \rho)_{\mathrm{b}}^{*}\left(p_{\mathrm{b}}^{*}\right)^{-1} \alpha\right\|=\left\|\rho_{\mathrm{b}}^{*} \alpha\right\|=\|\alpha\|=\left\|\left(p_{\mathrm{b}}^{*}\right)^{-1} \alpha\right\| .
$$


(4) By Proposition 2.1(1) and Remark 2.2 we have that

$$
\alpha=\sum_{i=1}^{n}\left(p_{i}\right)_{\mathrm{b}}^{*}\left(\left.\alpha\right|_{G_{i}}\right) .
$$

Then

$$
\rho^{*}(\alpha)=\sum_{i=1}^{n}\left(\rho_{i}\right)_{\mathrm{b}}^{*}\left(\left.\alpha\right|_{G_{i}}\right)
$$

so that, by (2.3) and $\alpha$-tightness of $\rho$, we have

$$
\sum_{i=1}^{n}\left\|\left.\alpha\right|_{G_{i}}\right\|=\|\alpha\|=\left\|\rho^{*}(\alpha)\right\|=\left\|\sum_{i=1}^{n}\left(\rho_{i}\right)_{\mathrm{b}}^{*}\left(\left.\alpha\right|_{G_{i}}\right)\right\| \leq \sum_{i=1}^{n}\left\|\left(\rho_{i}\right)_{\mathrm{b}}^{*}\left(\left.\alpha\right|_{G_{i}}\right)\right\| .
$$

The assertion now follows since

$$
\left\|\left(p_{i}\right)_{\mathrm{b}}^{*}\left(\left.\alpha\right|_{G_{i}}\right)\right\| \leq\left\|\left.\alpha\right|_{G_{i}}\right\| .
$$

2.3 Groups of type (RH). Let $G$ be a connected reductive Lie groups with compact center. Then $G=G_{c} \cdot G_{n c}$, where $G_{c}$ is the largest compact connected normal subgroup of $G$ and $G_{n c}$ is the product of all connected noncompact almost simple factors of $G$. Then $G_{n c}$ has finite center and $G_{c} \cap G_{n c}$ is finite.

Definition 2.7. (1) A Lie group $G$ is of Hermitian type if it is connected semisimple with finite center and no compact factors and its associated symmetric space is Hermitian.

(2) A group $G$ is of type ( $R H$ ) (reductive Hermitian) if it is a connected reductive Lie group with compact center such that $G_{n c}$ is of Hermitian type.

If $G$ is a group of type $(\mathrm{RH})$ and $\mathcal{X}$ is the symmetric space associated to $G_{n c}$ we have a homomorphism

$$
q: G \longrightarrow G_{\mathcal{X}}
$$

into $G_{\mathcal{X}}:=\operatorname{Isom}(\mathcal{X})^{\circ}$, which is surjective with compact kernel so that $G$ acts properly on $\mathcal{X}$.

Let $\mathcal{J}$ be the $G$-invariant complex structure on $\mathcal{X}$ and $g_{\mathcal{X}}$ the $G$-invariant Riemannian metric on $\mathcal{X}$, normalized so that the minimal holomorphic sectional curvature on every irreducible factor equals -1 . We denote by $\omega_{\mathcal{X}} \in \Omega^{2}(\mathcal{X})^{G}$ the $G$-invariant two-form

$$
\omega_{\mathcal{X}}(X, Y):=g_{\mathcal{X}}(X, \mathcal{J} Y)
$$

which is called the Kähler form of $\mathcal{X}$.

Choosing a base point $x_{0} \in \mathcal{X}$ any $G$-invariant two-form $\omega \in \Omega^{2}(\mathcal{X})^{G}$ gives rise to a continuous cocycle

$$
\begin{aligned}
& c_{\omega}: G \times G \times G \longrightarrow \mathbb{R} \\
&\left(g_{0}, g_{1}, g_{2}\right) \longmapsto \frac{1}{2 \pi} \int_{\Delta\left(g_{0} x_{0}, g_{1} x_{0}, g_{2} x_{0}\right)}^{\mathbb{R}} \omega,
\end{aligned}
$$

where $\Delta\left(g_{0} x_{0}, g_{1} x_{0}, g_{2} x_{0}\right)$ denotes a smooth oriented triangle with geodesic sides and vertices $g_{0} x_{0}, g_{1} x_{0}, g_{2} x_{0}$. Let $\kappa_{\omega}=\left[c_{\omega}\right] \in \mathrm{H}_{\mathrm{c}}^{2}(G, \mathbb{R})$ denote the corresponding continuous cohomology class; then the map $\omega \mapsto \kappa_{\omega}$ implements the van Est 
isomorphism $[\mathrm{E}]$

$$
\Omega^{2}(\mathcal{X})^{G} \cong \mathrm{H}_{\mathrm{c}}^{2}(G, \mathbb{R}) .
$$

It is well known that if $M$ is a connected simple Lie group with finite center, then

$$
\operatorname{dim} \mathrm{H}_{\mathrm{c}}^{2}(M, \mathbb{R})=0 \text { or } 1 ;
$$

in fact, the dimension is nonzero (hence 1) if and only if the associated symmetric space $\mathcal{M}$ carries a $M$-invariant complex structure and hence is Hermitian symmetric. Then $\Omega^{2}(\mathcal{M})^{M}=\mathbb{R} \omega_{\mathcal{M}}$, and with the above notation and normalizations we have

Theorem 2.8 [DT], [CØ]. If $\mathcal{M}$ is an irreducible Hermitian symmetric space we have that

$$
\frac{1}{2 \pi}\left|\sup _{\Delta \subset \mathcal{M}} \int_{\Delta} \omega_{\mathcal{M}}\right|=\frac{1}{2 \pi} \sup _{\Delta \subset \mathcal{M}} \int_{\Delta} \omega_{\mathcal{M}}=\frac{\mathrm{r}_{\mathcal{M}}}{2}
$$

where $\mathrm{r}_{\mathcal{M}}$ denotes the rank of $\mathcal{M}$.

In particular $c_{\omega_{\mathcal{M}}}$ defines a bounded class $\kappa_{M}^{\mathrm{b}} \in \mathrm{H}_{\mathrm{cb}}^{2}(M, \mathbb{R})$ which corresponds to $\kappa_{\omega} \in \mathrm{H}_{\mathrm{c}}^{2}(M, \mathbb{R})$ under the comparison map in $(2.1)$, and it was shown in [BuM1] that the comparison map

$$
c_{M}: \mathrm{H}_{\mathrm{cb}}^{2}(M, \mathbb{R}) \longrightarrow \mathrm{H}_{\mathrm{c}}^{2}(M, \mathbb{R})
$$

is an isomorphism in degree two. The following result for the canonical norm in continuous bounded cohomology could also in principle be deduced from [DT], [CØ].

Theorem 2.9. With the above notation and if $\mathcal{M}$ is irreducible, then

$$
\left\|\kappa_{M}^{\mathrm{b}}\right\|=\frac{\mathrm{r}_{\mathcal{M}}}{2} .
$$

Strictly speaking the concept of bounded continuous classes and their norms does not occur in [DT], [CØ]; what the authors show is that for a specific - and hence any - cocompact torsion-free lattice $\Gamma<M$, the singular bounded class in $\mathrm{H}_{\mathrm{b}}^{2}(\Gamma \backslash \mathcal{M})$ defined by integration of the Kähler form on straight simplices has Gromov norm $\pi \mathrm{r}_{\mathcal{M}}$. Using this and the isometric isomorphism between bounded singular cohomology of $\Gamma \backslash \mathcal{M}$ and bounded (group) cohomology of $\Gamma$, one could deduce the above theorem. We shall however give in the Appendix a direct proof which in particular avoids the construction of a lattice in $M$ with specific properties.

Let now $G$ be a group of type $(\mathrm{RH}), \mathcal{X}=\mathcal{X}_{1} \times \cdots \times \mathcal{X}_{n}$ a decomposition into irreducible factors, and $\omega_{\mathcal{X}, i}:=p_{i}^{*}\left(\omega_{\mathcal{X}_{i}}\right)$, where $p_{i}: \mathcal{X} \rightarrow \mathcal{X}_{i}$ is the projection onto the $i$-th factor. Then

$$
\left\{\omega_{\mathcal{X}, i} \in \Omega^{2}(\mathcal{X})^{G_{\mathcal{X}}}: 1 \leq i \leq n\right\}
$$

gives a basis of $\Omega^{2}(\mathcal{X})^{G \mathcal{X}}$ and, in view of the van Est isomorphism [E],

$$
\left\{\kappa_{\mathcal{X}, i}:=\kappa_{\omega_{\mathcal{X}, i}} \in \mathrm{H}_{\mathrm{c}}^{2}\left(G_{\mathcal{X}}, \mathbb{R}\right): 1 \leq i \leq n\right\}
$$

gives a basis of $\mathrm{H}_{\mathrm{c}}^{2}\left(G_{\mathcal{X}}, \mathbb{R}\right)$. Moreover, since it is the group $G_{\mathcal{X}}$ which acts effectively on $\mathcal{X}$, it is obvious that $\Omega^{2}(\mathcal{X})^{G}=\Omega^{2}(\mathcal{X})^{G \mathcal{X}}$, and hence the cohomology class defined by the cocycle $\mathrm{c}_{\omega}$ in $(2.5)$ can be thought of as a cohomology class in $\mathrm{H}_{\mathrm{c}}^{2}(G, \mathbb{R})$. Hence the map $q$ in (2.4) defines an isomorphism

$$
q^{*}: \mathrm{H}_{\mathrm{c}}^{2}\left(G_{\mathcal{X}}, \mathbb{R}\right) \longrightarrow \mathrm{H}_{\mathrm{c}}^{2}(G, \mathbb{R}),
$$


and we denote by

$$
\left\{\kappa_{G, i}:=q^{*}\left(\kappa_{\omega_{\mathcal{X}, i}}\right) \in \mathrm{H}_{\mathrm{c}}^{2}(G, \mathbb{R}): 1 \leq i \leq n\right\}
$$

the corresponding basis of $\mathrm{H}_{\mathrm{c}}^{2}(G, \mathbb{R})$.

If $\pi_{i}: G_{\mathcal{X}} \rightarrow G_{\mathcal{X}_{i}}$ denotes the projection onto the $i$-th factor, then we have that the analogous map to (2.9) in bounded cohomology

$$
q_{\mathrm{b}}^{*}: \mathrm{H}_{\mathrm{cb}}^{2}\left(G_{\mathcal{X}}, \mathbb{R}\right) \longrightarrow \mathrm{H}_{\mathrm{cb}}^{2}(G, \mathbb{R})
$$

and

$$
\begin{aligned}
\prod_{i=1}^{n} \mathrm{H}_{\mathrm{cb}}^{2}\left(G_{\mathcal{X}_{i}}, \mathbb{R}\right) & \longrightarrow \mathrm{H}_{\mathrm{cb}}^{2}\left(G_{\mathcal{X}}, \mathbb{R}\right) \\
\left(\kappa_{i}\right) & \longmapsto \sum_{i=1}^{n}\left(p_{i}\right)_{\mathrm{b}}^{*}\left(\kappa_{i}\right)
\end{aligned}
$$

are now isometric isomorphisms: for (2.11) this follows from Proposition 2.1(2) and the fact that ker $q$ is compact, and for (2.12) it follows already from Remark 2.2.

Let $\kappa_{\mathcal{X}, i}^{\mathrm{b}}$ be the bounded class of $G_{\mathcal{X}}$ defined by $c_{\omega_{\mathcal{X}, i}}=c_{\omega_{\mathcal{X}}} \circ p_{i}$; then it follows from the isomorphisms in (2.6) and (2.12) that

$$
\left\{\kappa_{\mathcal{X}, i}^{\mathrm{b}} \in \mathrm{H}_{\mathrm{cb}}^{2}\left(G_{\mathcal{X}}, \mathbb{R}\right): 1 \leq i \leq n\right\}
$$

gives a basis of $\mathrm{H}_{\mathrm{cb}}^{2}\left(G_{\mathcal{X}}, \mathbb{R}\right)$ and, analogously to before,

$$
\left\{\kappa_{G, i}^{\mathrm{b}}:=q_{\mathrm{b}}^{*}\left(\kappa_{\mathcal{X}, i}^{\mathrm{b}}\right) \in \mathrm{H}_{\mathrm{cb}}^{2}(G, \mathbb{R}): 1 \leq i \leq n\right\}
$$

a basis of $\mathrm{H}_{\mathrm{cb}}^{2}(G, \mathbb{R})$.

Thus if $\omega=\sum_{i=1}^{n} \lambda_{i} \omega_{\mathcal{X}, i}$ is any element in $\Omega^{2}(\mathcal{X})^{G}$ written in the above basis (2.7), then

$$
\kappa_{\omega}^{\mathrm{b}}=\sum_{i=1}^{n} \lambda_{i} \kappa_{G, i}^{\mathrm{b}}
$$

is the bounded class in $\mathrm{H}_{\mathrm{cb}}^{2}(G, \mathbb{R})$ defined by $c_{\omega}=\sum_{i=1}^{n} \lambda_{i} c_{\omega_{\mathcal{X}, i}}$ and corresponding to $\omega$ under the isomorphism $\Omega^{2}(\mathcal{X})^{G} \rightarrow \mathrm{H}_{\mathrm{cb}}^{2}(G, \mathbb{R})$. Moreover applying the isometric isomorphism in (2.12) and Theorem 2.9 we have that

$$
\left\|\kappa_{\omega}^{\mathrm{b}}\right\|=\sum_{i=1}^{n}\left|\lambda_{i}\right|\left\|\kappa_{G, i}^{\mathrm{b}}\right\|=\sum_{i=1}^{n}\left|\lambda_{i}\right| \frac{\mathrm{r}_{\mathcal{X}_{i}}}{2}
$$

and in particular

$$
\left\|\kappa_{G}^{\mathrm{b}}\right\|=\frac{\mathrm{r}_{\mathcal{X}}}{2} .
$$

With the same notation we have

Proposition 2.10. For any Hermitian symmetric space $\mathcal{X}$ we have that

$$
\frac{1}{2 \pi} \sup _{\Delta \subset \mathcal{X}}\left|\int_{\Delta} \omega\right|=\frac{1}{2 \pi} \sup _{\Delta \subset \mathcal{X}} \int_{\Delta} \omega=\sum_{i=1}^{n}\left|\lambda_{i}\right| \frac{\mathrm{r}_{\mathcal{X}_{i}}}{2},
$$

where $\Delta \subset \mathcal{X}$ runs through all smooth triangles with geodesic sides in $\mathcal{X}$. 
Proof. We assume here Theorem 2.8 and we focus on the nonirreducible case. If $\omega=\sum_{i=1}^{n} \lambda_{i} \omega_{\mathcal{X}, i}$, then

$$
\int_{\Delta} \omega=\sum_{i=1}^{n} \lambda_{i} \int_{\Delta} \omega_{\mathcal{X}, i}=\sum_{i=1}^{n} \lambda_{i} \int_{p_{i}(\Delta)} \omega_{\mathcal{X}_{i}}
$$

so that

$$
\left|\frac{1}{2 \pi} \int_{\Delta} \omega\right|=\frac{1}{2 \pi}\left|\sum_{i=1}^{n} \lambda_{i} \int_{p_{i}(\Delta)} \omega_{\mathcal{X}_{i}}\right| \leq \frac{1}{2 \pi} \sum_{i=1}^{n}\left|\lambda_{i}\right|\left|\int_{p_{i}(\Delta)} \omega_{\mathcal{X}_{i}}\right| \leq \sum_{i=1}^{n}\left|\lambda_{i}\right| \frac{\mathrm{r}_{\mathcal{X}_{i}}}{2},
$$

where we used Theorem 2.8 in the last inequality. For the opposite inequality, let $\epsilon>0$ and $\Delta_{i} \subset \mathcal{X}_{i}$ be smooth triangles with geodesic sides such that

$$
\int_{\Delta_{i}} \omega_{\mathcal{X}_{i}} \geq \pi \mathrm{r}_{\mathcal{X}_{i}}-\epsilon
$$

More precisely let $\sigma_{i}^{+}: \Delta \rightarrow \mathcal{X}_{i}$ be a parametrization of $\Delta_{i}$ with geodesic sides, and $\sigma_{i}^{-}$be the parametrization of $\Delta_{i}$ with the opposite orientation. Then let $\Delta_{\mathcal{X}}$ be the image of the map

$$
\begin{array}{rlc}
\sigma: \Delta & \longrightarrow & \mathcal{X} \\
t & \longmapsto & \left(\sigma_{1}^{\eta_{1}}(t), \ldots, \sigma_{n}^{\eta_{n}}(t)\right),
\end{array}
$$

where $\eta_{i}=\operatorname{sign}\left(\lambda_{i}\right)$. Then

$$
\int_{\Delta_{\mathcal{X}}} \omega_{\mathcal{X}, i}=\int_{\sigma_{i}^{\eta_{i}}} \omega_{\mathcal{X}_{i}}
$$

and

$$
\int_{\Delta_{\mathcal{X}}} \omega=\sum_{i=1}^{n} \lambda_{i} \int_{\sigma_{i}^{\eta_{i}}} \omega_{\mathcal{X}_{i}}=\sum_{i=1}^{n}\left|\lambda_{i}\right| \int_{\Delta_{i}} \omega_{\mathcal{X}_{i}} \geq \pi\left(\sum_{i=1}^{n}\left|\lambda_{i}\right| \mathrm{r}_{\mathcal{X}_{i}}\right)-\epsilon \sum_{i=1}^{n}\left|\lambda_{i}\right| .
$$

Since this holds for any $\epsilon>0$ the proof is complete.

Definition 2.11. Let $L$ be a locally compact second countable topological group and $G$ a group of type (RH). A continuous homomorphism $\rho: L \rightarrow G$ is said to be tight, if $\rho$ is $\kappa_{G}^{\mathrm{b}}$-tight, that is if

$$
\left\|\rho^{*}\left(\kappa_{G}^{\mathrm{b}}\right)\right\|=\left\|\kappa_{G}^{\mathrm{b}}\right\|
$$

Proposition 2.12. Let $H, G$ be Lie groups of type $(R H), \mathcal{X}$ the symmetric space associated to $G$ and $\mathcal{Y}=\mathcal{Y}_{1} \times \cdots \times \mathcal{Y}_{n}$ the symmetric space associated to $H$, where the $\mathcal{Y}_{i}$ 's are irreducible. Let $\rho: H \rightarrow G$ be a homomorphism and assume that $\rho^{*}\left(\kappa_{G}^{\mathrm{b}}\right)=\sum_{i=1}^{n} \lambda_{i} \kappa_{H, i}^{\mathrm{b}}$. Then $\rho$ is tight if and only if $\mathrm{r}_{\mathcal{X}}=\sum_{i=1}^{n}\left|\lambda_{i}\right| \mathrm{r}_{\mathcal{Y}_{i}}$.

Proof. We have by $(2.15)$

$$
\left\|\rho^{*}\left(\kappa_{G}^{\mathrm{b}}\right)\right\|=\sum_{i=1}^{n}\left|\lambda_{i}\right|\left\|\kappa_{H, i}^{\mathrm{b}}\right\|,
$$

and since $\left\|\kappa_{G}^{\mathrm{b}}\right\|=\mathrm{r}_{\mathcal{X}} / 2$ (by (2.16)) and $\left\|\kappa_{H, i}^{\mathrm{b}}\right\|=\mathrm{r}_{\mathcal{Y}_{i}} / 2$ (by Theorem 2.9), the assertion follows immediately. 
2.4 Tight maps. Let now $f: \mathcal{X}_{1} \rightarrow \mathcal{X}_{2}$ be a totally geodesic map, where $\mathcal{X}_{1}, \mathcal{X}_{2}$ are Hermitian symmetric spaces of noncompact type.. Recall that this means that given any geodesic $c: \mathbb{R} \rightarrow \mathcal{X}_{1}$, the path $f \circ c: \mathbb{R} \rightarrow \mathcal{X}_{2}$ is a geodesic, possibly not parametrized by arclength; in fact, $f \circ c$ might be the constant map. At any rate, for every triangle $\Delta_{1} \subset \mathcal{X}_{1}$ with geodesic sides, $f\left(\Delta_{1}\right) \subset \mathcal{X}_{2}$ is so as well, and hence

$$
\sup _{\Delta_{1} \subset \mathcal{X}_{1}} \int_{\Delta_{1}} f^{*}\left(\omega_{\mathcal{X}_{2}}\right) \leq \sup _{\Delta_{2} \subset \mathcal{X}_{2}} \int_{\Delta_{2}} \omega_{\mathcal{X}_{2}}
$$

where the supremum on each side is taken over all triangles with geodesic sides.

Definition 2.13. (1) A totally geodesic map $f: \mathcal{X}_{1} \rightarrow \mathcal{X}_{2}$ is tight if equality holds in (2.17).

(2) We say that a subsymmetric space $\mathcal{Y} \subset \mathcal{X}_{2}$ is tightly embedded if the inclusion map is tight.

Proposition 2.14. Let $f: \mathcal{X}_{1} \rightarrow \mathcal{X}_{2}$ be a totally geodesic map and $\mathcal{Y} \subset f\left(\mathcal{X}_{1}\right)$ a subsymmetric space.

(1) The map $f$ is tight if and only if the subsymmetric space $f\left(\mathcal{X}_{1}\right)$ is tightly embedded in $\mathcal{X}_{2}$.

(2) If $\mathcal{Y}$ is tightly embedded in $\mathcal{X}_{2}$, then $f$ is tight.

Proof. For the first assertion it suffices to observe that every triangle in $f\left(\mathcal{X}_{1}\right)$ with geodesic sides is the image of a triangle in $\mathcal{X}_{1}$ with geodesic sides. This implies that

$$
\sup _{\Delta_{1} \subset \mathcal{X}_{1}} \int_{\Delta_{1}} f^{*}\left(\omega_{\mathcal{X}_{2}}\right)=\sup _{\Delta_{2} \subset f\left(\mathcal{X}_{1}\right)} \int_{\Delta_{2}} \omega_{\mathcal{X}_{2}}
$$

which shows the first assertion.

The second assertion follows immediately from the above and the inequalities

$$
\sup _{\Delta \subset \mathcal{Y}} \int_{\Delta} \omega \mathcal{X}_{2} \leq \sup _{\Delta_{1} \subset f\left(\mathcal{X}_{1}\right)} \int_{\Delta_{1}} \omega_{\mathcal{X}_{2}} \leq \sup _{\Delta_{2} \subset \mathcal{X}_{2}} \int_{\Delta_{2}} \omega_{\mathcal{X}_{2}} .
$$

Given a homomorphism $\rho: G_{1} \rightarrow G_{2}$ of Lie groups of type (RH), let $x_{1} \in \mathcal{X}_{1}$ be a base point, $K_{1}=\operatorname{Stab}_{G_{1}}\left(x_{1}\right)$ the corresponding maximal compact subgroup and $x_{2} \in \mathcal{X}_{2}$ a point such that $\rho\left(K_{1}\right)\left(x_{2}\right)=x_{2}$. Then $\rho$ gives rise to a map

$$
f: \mathcal{X}_{1} \longrightarrow \mathcal{X}_{2}
$$

defined by $f\left(g x_{1}\right):=\rho(g) x_{2}$, which is $\rho$-equivariant and totally geodesic.

LemMa 2.15. The diagram

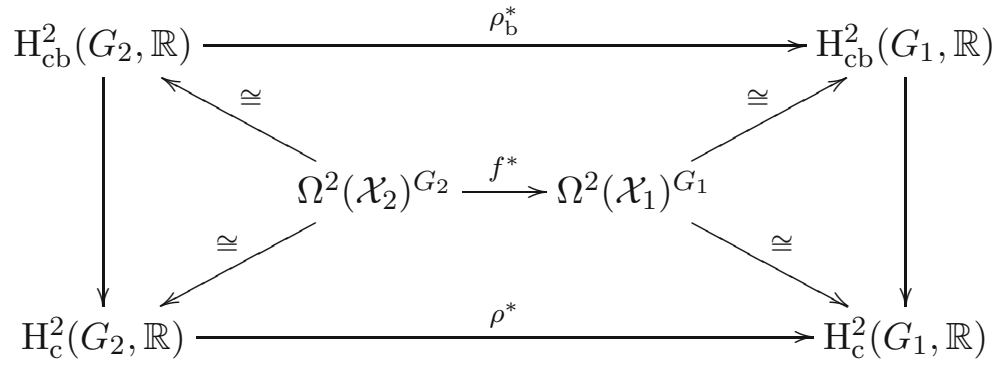

commutes. 
Proof. Let $\omega \in \Omega^{2}\left(\mathcal{X}_{2}\right)^{G_{2}}$. Using the points $x_{1} \in \mathcal{X}_{1}$ and $x_{2}=f\left(x_{1}\right) \in \mathcal{X}_{2}$ in the construction of the cocycles we have

$$
c_{f^{*}(\omega)}=c_{\omega} \circ \rho .
$$

Corollary 2.16. In the above situation the following are equivalent:

(1) The homomorphism $\rho: G_{1} \rightarrow G_{2}$ is tight.

(2) The totally geodesic map $f: \mathcal{X}_{1} \rightarrow \mathcal{X}_{2}$ is tight.

Proof. By Lemma 2.15 we have that $\rho^{*}\left(\kappa_{G_{2}}^{\mathrm{b}}\right)=\kappa_{f^{*}\left(\omega_{\mathcal{X}_{2}}\right)}^{\mathrm{b}}$. Thus, applying Proposition 2.10 we have that

$$
\left\|\rho^{*}\left(\kappa_{G_{2}}^{\mathrm{b}}\right)\right\|=\left\|\kappa_{f^{*}\left(\omega_{\mathcal{X}_{2}}\right)}^{\mathrm{b}}\right\|=\frac{1}{2 \pi} \sup _{\Delta_{1} \subset \mathcal{X}_{1}} \int_{\Delta_{1}} f^{*} \omega_{\mathcal{X}_{2}}
$$

and

$$
\left\|\kappa_{G_{2}}^{\mathrm{b}}\right\|=\frac{1}{2 \pi} \sup _{\Delta_{2} \subset \mathcal{X}_{2}} \int_{\Delta_{2}} \omega_{\mathcal{X}_{2}}
$$

from which the equivalence follows readily.

From Corollary 2.16 and Proposition 2.12 we immediately deduce the following: Corollary 2.17. Let $H, G$ be Lie groups of type $(R H)$ with associated Hermitian symmetric spaces $\mathcal{Y}$ and $\mathcal{X}, \rho: H \rightarrow G$ a homomorphism and $f: \mathcal{Y} \rightarrow \mathcal{X}$ a $\rho$ equivariant totally geodesic map. Let $\mathcal{Y}=\mathcal{Y}_{1} \times \cdots \times \mathcal{Y}_{n}$ be the decomposition into irreducible factors and suppose that $f^{*}\left(\omega_{\mathcal{X}}\right)=\sum_{i=1}^{n} \lambda_{i} \omega_{\mathcal{Y}, i}$. The totally geodesic map $f: \mathcal{Y} \rightarrow \mathcal{X}$ is tight if and only if $\mathrm{r}_{\mathcal{X}}=\sum_{i=1}^{n}\left|\lambda_{i}\right| \mathrm{r}_{\mathcal{Y}_{i}}$.

Before stating the next corollary let us recall the following:

DeFinition 2.18. (1) A maximal polydisk in $\mathcal{X}$ is the image of a totally geodesic and holomorphic embedding $t: \mathbb{D}^{\mathrm{r} \mathcal{X}} \rightarrow \mathcal{X}$ of a product of $r_{\mathcal{X}}$ Poincaré disks.

(2) A diagonal disk in $\mathcal{X}$ is the image of the diagonal $\Delta(\mathbb{D}) \subset \mathbb{D}^{r \mathcal{X}}$ under an embedding $t: \mathbb{D}^{\mathrm{r} \mathcal{X}} \rightarrow \mathcal{X}$ of $\mathbb{D}^{\mathrm{r} \mathcal{X}}$ as a maximal polydisk. In particular $d:=t \circ \Delta:$ $\mathbb{D} \rightarrow \mathcal{X}$ is a totally geodesic and holomorphic embedding.

Maximal polydisks arise as complexifications of maximal flats in $\mathcal{X}$, and hence are conjugate under $G_{\mathcal{X}}$. Moreover, with the normalization chosen in section 2.3 the embedding $t: \mathbb{D}^{r \mathcal{X}} \rightarrow \mathcal{X}$ is isometric. In fact one can say more, as we have

Lemma 2.19. A metric on an Hermitian symmetric space $\mathcal{X}$ is normalized if and only if every maximal polydisk $f: \mathbb{D}^{\mathrm{r}} \mathcal{X} \rightarrow \mathcal{X}$ is isometrically embedded.

Proof. If $\mathcal{X}$ is irreducible, it follows from the computation in [C $\varnothing$, p. 273-274], that the holomorphic sectional curvature is minimal at $u \in T_{x} \mathcal{X}$ if and only if the complex geodesic obtained by $u$ is the image of a factor of a maximal polydisk $t: \mathbb{D}^{\mathrm{r}} \mathcal{X} \rightarrow \mathcal{X}$. The general case follows immediately.

Lemma 2.19 has the following useful consequence:

Corollary 2.20. Let $\mathcal{Y} \subset \mathcal{X}$ be a Hermitian subsymmetric space of the same rank as $\mathcal{X}$. Then the restriction to $\mathcal{Y}$ of the normalized metric on $\mathcal{X}$ is the normalized metric on $\mathcal{Y}$. 
Proof. Indeed, every maximal polydisk $t: \mathbb{D}^{\mathrm{r} \mathcal{Y}} \rightarrow \mathcal{Y}$ in $\mathcal{Y}$ is a maximal polydisk in $\mathcal{X}$.

Definition 2.21. A Hermitian symmetric space $\mathcal{X}$ is said to be of tube type if $\mathcal{X}$ is biholomorphically equivalent to a tube domain of the form

$$
\{v+i u \mid v \in V, u \in \Omega\} \subset V \oplus i V,
$$

where $V$ is a real vector space and $\Omega \subset V$ is a proper open cone.

Every Hermitian symmetric space $\mathcal{X}$ contains maximal (with respect to the inclusion) subdomains $T$ of tube type which are of the same rank as $\mathcal{X}$, holomorphically embedded and conjugate under $G_{\mathcal{X}}$. Moreover, the embedding $T \subset \mathcal{X}$ is always isometric (see Corollary 2.20).

Corollary 2.22. (1) Let $f: \mathcal{Y} \rightarrow \mathcal{X}$ be a holomorphic and isometric embedding. Then $f$ is tight if and only if $\mathrm{r}_{\mathcal{Y}}=\mathrm{r}_{\mathcal{X}}$.

(2) Maximal polydisks $t: \mathbb{D}^{\mathrm{r} \mathcal{X}} \rightarrow \mathcal{X}$ are tight.

(3) Diagonal disks $d: \mathbb{D} \rightarrow \mathcal{X}$ are tight.

(4) Maximal tube type subdomains $T \subset \mathcal{X}$ are tight.

Proof. If $f: \mathcal{Y} \rightarrow \mathcal{X}$ is holomorphic and isometric, then $f^{*}\left(\omega_{\mathcal{X}}\right)=\omega_{\mathcal{Y}}=\sum_{i=1}^{n} \omega_{\mathcal{Y}, i}$, so (1) follows from the fact that $\mathrm{r}_{\mathcal{Y}}=\sum_{i=1}^{n} \mathrm{r}_{\mathcal{Y}_{i}}$ and Proposition 2.12.

Then (2) and (4) follow at once from (1) since the embeddings $t: \mathbb{D}^{\mathrm{r} \mathcal{X}} \rightarrow \mathcal{X}$ and $T \subset \mathcal{X}$ are holomorphic and isometric.

To see $(3)$, observe that since $t$ is a holomorphic isometry, then $t^{*}\left(\omega_{\mathcal{X}}\right)=\omega_{\mathbb{D}^{\mathrm{X}} \mathcal{X}}$; moreover, an easy verification shows that $\Delta^{*}\left(\omega_{\mathbb{D}^{r} \mathcal{X}}\right)=\mathrm{r}_{\mathcal{X}} \omega_{\mathbb{D}}$. It follows then that $d^{*}\left(\omega_{\mathcal{X}}\right)=\mathrm{r}_{\mathcal{X}} \omega_{\mathbb{D}}$, so that the assertion follows from Proposition 2.12.

Further examples of tight maps and tight homomorphisms will be discussed in section 8.1.

\section{Kähler Classes and the Shilov Boundary}

In this section we collect the facts from the geometry of Hermitian symmetric spaces, some of which are of independent interest, needed for our purpose. Those concerning the geometry of triangles are due, in the context of irreducible domains, to Clerc and Ørsted [CØ]; we present also here the necessary - easy - extensions to general domains.

Let $\mathcal{X}$ be a Hermitian symmetric space of noncompact type with a fixed $G_{\mathcal{X}}$ invariant complex structure $\mathcal{J}$. Fix a maximal compact subgroup $K=\operatorname{Stab}_{G_{\mathcal{X}}}\left(x_{0}\right)$, where $x_{0} \in \mathcal{X}$ is some base point. Let $\mathfrak{g}=\mathfrak{k} \oplus \mathfrak{p}$ be the corresponding Cartan decomposition, where $\mathfrak{g}=\operatorname{Lie}\left(G_{\mathcal{X}}\right)$ and $\mathfrak{k}=\operatorname{Lie}(K)$. There exists a unique element $Z_{\mathcal{J}}$ in the center $\mathcal{Z}(\mathfrak{k})$ of $\mathfrak{k}$ such that $\left.\operatorname{ad}\left(Z_{\mathcal{J}}\right)\right|_{\mathfrak{p}}$ induces the complex structure $\mathcal{J}$ under the identification $\mathfrak{p} \cong T_{x_{0}} \mathcal{X}$. The complexification $\mathfrak{g} \mathbb{C}$ of $\mathfrak{g}$ splits into eigenspaces of $\operatorname{ad}\left(Z_{\mathcal{J}}\right)$ as

$$
\mathfrak{g}_{\mathbb{C}}=\mathfrak{k}_{\mathbb{C}} \oplus \mathfrak{p}_{+} \oplus \mathfrak{p}_{-} .
$$


The Hermitian symmetric space $\mathcal{X}$ can be realized as a bounded symmetric domain

$$
\mathcal{D} \subset \mathfrak{p}_{+} \cong \mathbb{C}^{N}
$$

Let us describe the structure of $\mathcal{D}$ more explicitly. Let us fix $\mathfrak{h} \subset \mathfrak{k}$ a maximal Abelian subalgebra. Then $\mathcal{Z}(\mathfrak{k}) \subset \mathfrak{h}$ and $\mathfrak{h}_{\mathbb{C}}$ is a maximal Abelian subalgebra, indeed a Cartan subalgebra of $\mathfrak{g}_{\mathbb{C}}$. The set $\Psi=\Psi\left(\mathfrak{g}_{\mathbb{C}}, \mathfrak{h}_{\mathbb{C}}\right)$ of roots of $\mathfrak{h}_{\mathbb{C}}$ in $\mathfrak{g}_{\mathbb{C}}$ decomposes compatibly with the decomposition of $\mathfrak{g}_{\mathbb{C}}$ in (3.1) as

$$
\Psi=\Psi^{\mathfrak{k}_{\mathbb{C}}} \cup \Psi^{\mathfrak{p}_{+}} \cup \Psi^{\mathfrak{p}_{-}},
$$

here $\Psi^{\mathfrak{l}}:=\left\{\alpha \in \Psi \mid\right.$ the root space $\left.\mathfrak{g}_{\alpha} \subset \mathfrak{l}\right\}$. One can choose an ordering $\Psi=\Psi_{+} \cup \Psi_{-}$ such that $\Psi^{\mathfrak{p}_{ \pm}} \subset \Psi_{ \pm}$.

To every root $\alpha \in \Psi$ we associate a three dimensional simple subalgebra

$$
\mathfrak{g}_{[\alpha]}=\mathbb{C} H_{\alpha} \oplus \mathbb{C} E_{\alpha} \oplus \mathbb{C} E_{-\alpha},
$$

where $H_{\alpha} \in \mathfrak{h}_{\mathbb{C}}$ is the unique element determined by $\alpha(H)=2 \frac{\mathfrak{B}\left(H, H_{\alpha}\right)}{\mathfrak{B}\left(H_{\alpha}, H_{\alpha}\right)}$ for all $H \in \mathfrak{h}_{\mathbb{C}}$ and $\mathfrak{B}$ is the Killing form on $\mathfrak{g}_{\mathbb{C}}$. The elements $E_{\alpha}, E_{-\alpha}$ are the elements of $\mathfrak{g}_{ \pm \alpha}$ satisfying the relation $\left[E_{\alpha}, E_{-\alpha}\right]=H_{\alpha}$ and $\tau\left(E_{\alpha}\right)=-E_{-\alpha}$, where $\tau$ is the complex conjugation of $\mathfrak{g}_{\mathbb{C}}$ with respect to the compact real form $g_{U}=\mathfrak{k} \oplus i \mathfrak{p}$. Then $\mathfrak{p}_{+}=\sum_{\alpha \in \Psi^{\mathfrak{p}}+} \mathbb{C} E_{\alpha}$ and the vectors $X_{\alpha}=E_{\alpha}+E_{-\alpha}, Y_{\alpha}=i\left(E_{\alpha}-E_{-\alpha}\right), \alpha \in \Psi^{\mathfrak{p}_{+}}$, form a real basis of $\mathfrak{p}$.

Two roots $\alpha, \beta \in \Psi$ are called strongly orthogonal if neither $\alpha+\beta$ nor $\alpha-\beta$ is a root. By a theorem of Harish-Chandra there exists a maximal set $\Lambda=$ $\left\{\gamma_{1}, \ldots, \gamma_{r}\right\} \subset \Psi^{\mathfrak{p}_{+}}$of $r=\mathrm{r}_{\mathcal{D}}$ strongly orthogonal roots. The associated vectors $X_{\gamma_{j}} \in \mathfrak{p}$ span a maximal Abelian subspace $\mathfrak{a}$ of $\mathfrak{p}$ over $\mathbb{R}$. The bounded symmetric domain $\mathcal{D}$ admits the following description

$$
\mathcal{D}=\left\{\operatorname{Ad}(k) \sum_{j=1}^{r} \tanh \left(t_{j}\right) E_{\gamma_{j}}: k \in K, t_{j} \in \mathbb{R}\right\} \subset \mathfrak{p}_{+} ;
$$

moreover, we call

$$
\mathcal{P}_{0}=\left\{\operatorname{Ad}(k) \sum_{j=1}^{r} \tanh \left(t_{j}\right) E_{\gamma_{j}}: k \in \exp (\mathfrak{h}), t_{j} \in \mathbb{R}\right\} \subset \mathcal{D}
$$

the standard maximal polydisk and

$$
\Delta_{0}=\left\{\operatorname{Ad}(k) \sum_{j=1}^{r} \tanh (t) E_{\gamma_{j}}: k \in \mathcal{Z}(K), t \in \mathbb{R}\right\} \subset \mathcal{P}_{0},
$$

the standard diagonal disk. With the explicit description of $\mathcal{D}$ we define the (normalized) Bergmann kernel

$$
k_{\mathcal{D}}: \mathcal{D} \times \mathcal{D} \rightarrow \mathbb{C}^{\times}
$$

by

$$
k_{\mathcal{D}}(z, w)=h_{\mathcal{D}}(z, w)^{-2}
$$

where $h_{\mathcal{D}}(z, w)$ is the polarization of the unique $K$-invariant polynomial $h$ on $\mathfrak{p}_{+}$ such that

$$
h\left(\sum_{j=1}^{r} s_{j} E_{\gamma_{j}}\right)=\prod_{j=1}^{r}\left(1-s_{j}^{2}\right)
$$


The (normalized) Bergmann kernel is continuous on $\mathcal{D}^{2}$ and gives rise to a Riemannian metric $g_{\mathcal{D}}$, called the (normalized) Bergmann metric on $\mathcal{D}$, which has minimal holomorphic sectional curvature -1 : this holds in the irreducible case by $[\mathrm{C} \varnothing,(1.2)]$ and follows for the general case by the naturality under product of the normalized metric. Let us observe that the Bergmann metric and the normalized Bergmann metric are equivalent: indeed for an irreducible domain they are proportional - the proportionality factor however depends on the domain, see [CØ, (1.2)] for the precise value - and the Bergmann and normalized Bergmann metrics behave functorially with respect to taking products.

The Kähler form given by

$$
\omega_{\mathcal{D}}=i \partial \bar{\partial} \log k_{\mathcal{D}}(z, z)
$$

corresponds to $\omega_{\mathcal{X}}$ under the isomorphism $\mathcal{X} \rightarrow \mathcal{D}$.

Lemma 3.1. Let $\mathcal{D} \subset \mathbb{C}^{N}$ be a bounded symmetric domain with Riemannian distance $d_{\mathcal{D}}(\cdot, \cdot)$. Then there exists a constant $c=c(\mathcal{D})$ such that for all $x, y \in \mathcal{D}$

$$
d_{\mathcal{D}}(x, y) \geq c\|x-y\|_{\text {eucl }},
$$

where $\|\cdot\|_{\text {eucl }}$ denotes the Euclidean norm on $\mathbb{C}^{N}$.

Proof. Using the observation above, it suffices to show the lemma for the distance coming from the Bergmann metric.

Let $b_{\mathcal{D}}$ be this metric, then at every $z \in \mathcal{D}$ we have

$$
\left(b_{\mathcal{D}}\right)_{z}(\cdot, \cdot)=\left\langle\cdot, \operatorname{Ad} \mathcal{K}(z, z)^{-1} \cdot\right\rangle,
$$

where $\langle\cdot, \cdot\rangle$ is the Hermitian form on $\mathfrak{p}_{+}$coming from the Killing form and $\mathcal{K}$ is the kernel function defined on an open subset of $\mathfrak{p}_{+} \times \mathfrak{p}_{+}$with values in the complexification $K_{\mathbb{C}}$ of the maximal compact subgroup (for definition and details see [S2, §5.6 and Prop. 6.2]).

We now need to estimate the eigenvalues of $\operatorname{Ad} \mathcal{K}(z, z)$. Writing $z=\operatorname{Ad}(k) z_{1}$ and observing that

$$
\operatorname{Ad} \mathcal{K}(z, z)=\operatorname{Ad}(k) \operatorname{Ad} \mathcal{K}\left(z_{1}, z_{1}\right) \operatorname{Ad}(k)^{-1},
$$

we may assume that

$$
z_{1}=\sum_{j=1}^{r_{\mathcal{D}}} \xi_{j} E_{\gamma_{j}} .
$$

An explicit calculation (see e.g. [S2, p.71]) shows that the eigenvalues of $\operatorname{Ad} \mathcal{K}\left(z_{1}, z_{1}\right)$ on the root space $\mathfrak{g}_{\alpha}$ for $\alpha \in \Psi^{\mathfrak{p}_{+}}$are given by

$$
\begin{aligned}
& \left(1-\left|\xi_{j}\right|^{2}\right)^{2}, \\
& \left(1-\left|\xi_{j}\right|^{2}\right)\left(1-\left|\xi_{k}\right|^{2}\right), \\
& \left(1-\left|\xi_{j}\right|^{2}\right), \text { or } \\
& \left(1-\left|\xi_{j}\right|^{2}\right)\left(1+\left|\xi_{k}\right|^{2}\right)+\left|\xi_{k}\right|^{4},
\end{aligned}
$$

where $1 \leq j \neq k \leq \mathrm{r}_{\mathcal{X}}$. In particular, since $0 \leq\left|\xi_{j}\right|<1$ every eigenvalue of $\operatorname{Ad} \mathcal{K}(z, z)^{-1}$ is greater than $1 / 3$ and the claim follows. 
3.1 Shilov boundary. We shall denote by $G_{\mathcal{D}}$ the connected component of the group $\operatorname{Aut}(\mathcal{D})$ of holomorphic automorphisms of $\mathcal{D}$. When $\mathcal{D}^{\prime} \subset \mathcal{D}$ is a Hermitian symmetric subspace, we shall denote by $A_{\mathcal{D}^{\prime}}$ the subgroup of $G_{\mathcal{D}}$ of Hermitian type associated to $\mathcal{D}^{\prime}$; in fact, $A_{\mathcal{D}^{\prime}}$ is the product of the noncompact connected almost simple factors of the reductive subgroup $\mathcal{N}_{G_{\mathcal{D}}}\left(\mathcal{D}^{\prime}\right)$, where if $E \subset \overline{\mathcal{D}}$ is any subset, we define

$$
\mathcal{N}_{G_{\mathcal{D}}}(E):=\left\{g \in G_{\mathcal{D}}: g(E)=E\right\} .
$$

The closure $\overline{\mathcal{D}}$ contains a unique closed $G_{\mathcal{D}}$-orbit which is the Shilov boundary $\check{S}_{\mathcal{D}}$ of $\mathcal{D}$; more precisely, the Shilov boundary $\check{S}_{\mathcal{D}}$ is the $G_{\mathcal{D}}$ orbit of the point $\sum_{j=1}^{r} E_{\gamma_{j}} \subset \mathfrak{p}_{+}$, where $E_{\gamma_{j}}$ are the root vectors associated to strongly orthogonal roots $\gamma_{j} \in \Lambda$ (see (3.2)), and can hence be realized as $G_{\mathcal{D}} / Q$, where $Q$ is the stabilizer in $G_{\mathcal{D}}$ of $\sum_{j=1}^{r} E_{\gamma_{j}}$. In particular, if $\mathcal{D}$ is irreducible, then $Q$ is a maximal parabolic subgroup in $G_{\mathcal{D}}$.

Lemma 3.2. (1) Let $\mathcal{D}=\mathcal{D}_{1} \times \cdots \times \mathcal{D}_{n}$ be a decomposition into irreducible factors. Then the Shilov boundary $\check{S}_{\mathcal{D}}$ of $\mathcal{D}$ is the product $\check{S}_{\mathcal{D}_{1}} \times \cdots \times \check{S}_{\mathcal{D}_{n}}$ of the Shilov boundaries of the irreducible factors.

(2) If $\mathcal{P}$ is any maximal polydisk and $\Delta \subset \mathcal{P}$ is any diagonal disk, then $\check{S}_{\Delta} \subset \check{S}_{\mathcal{P}} \subset \check{S}_{\mathcal{D}}$.

(3) If $\mathcal{D}^{\prime} \subset \mathcal{D}$ is a Hermitian symmetric subspace with $\mathrm{r}_{\mathcal{D}^{\prime}}=\mathrm{r}_{\mathcal{D}}$, then $\check{S}_{\mathcal{D}^{\prime}} \subset \check{S}_{\mathcal{D}}$.

Proof. (1) This first assertion follows from the characterization of $\check{S}_{\mathcal{D}}$ as the unique

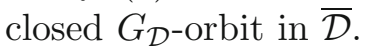

(2) To see the second assertion observe that since all maximal polydisks (and their diagonal disks) are conjugate by $G_{\mathcal{D}}$, it suffices to show the assertion for the standard maximal polydisk $\mathcal{P}_{0}$. First it is obvious that $\check{S}_{\Delta_{0}} \subset \check{S}_{\mathcal{P}_{0}}$. Then let $A_{\mathcal{P}_{0}}$ be the subgroup of Hermitian type of $G_{\mathcal{D}}$ associated to $\mathcal{P}_{0}$; clearly the vector $\sum_{j=1}^{r} E_{\gamma_{j}}$ is contained in $\check{S}_{\mathcal{P}_{0}}$ and hence its $A_{\mathcal{P}_{0}}$-orbit is contained in its $G_{\mathcal{D}}$-orbit, which implies that $\check{S}_{\mathcal{P}_{0}} \subset \check{S}_{\mathcal{D}}$ and hence the second assertion.

(3) Finally, let $\mathcal{P} \subset \mathcal{D}^{\prime}$ be a maximal polydisk and let $A_{\mathcal{D}^{\prime}}$ be the subgroup of Hermitian type of $G_{\mathcal{D}}$ associated to $\mathcal{D}^{\prime}$. Then $\mathcal{P}$ is maximal in $\mathcal{D}$ as well and hence, by $(2), \check{S}_{\mathcal{P}} \subset \check{S}_{\mathcal{D}^{\prime}}$. This, together with the obvious inclusion $A_{\mathcal{D}^{\prime}}\left(\check{S}_{\mathcal{P}}\right) \subset G_{\mathcal{D}}\left(\check{S}_{\mathcal{P}}\right)$ implies that $\check{S}_{\mathcal{D}^{\prime}} \subset \check{S}_{\mathcal{D}}$.

The relationship between the geodesic ray compactification $\mathcal{D}(\infty)$ of $\mathcal{X}$ and the boundary $\partial \mathcal{D}$ of the domain $\mathcal{D}$ is far from being simple. For example, a point in $\mathcal{D}(\infty)$ does not uniquely determine one in $\partial \mathcal{D}$; this is however true if the endpoint of a geodesic ray lies in the Shilov boundary. In fact we have

Lemma 3.3 [L, Th. 9.11]. Let $z \in \check{S}_{\mathcal{D}}$ be a point in the Shilov boundary of $\mathcal{D}$ and let $\eta_{1}, \eta_{2}:[0, \infty) \rightarrow \mathcal{D}$ be geodesic rays such that

(1) $\lim _{t \rightarrow \infty} \eta_{1}(t)=z$, and

(2) $\sup _{t \geq 0} d_{\mathcal{D}}\left(\eta_{1}(t), \eta_{2}(t)\right)<\infty$.

Then $\lim _{t \rightarrow \infty} \eta_{2}(t)=z$.

Sketch of the proof. We can assume that the bounded symmetric domain is irreducible and that $z=e Q \in \check{S}=G_{\mathcal{D}} / Q$. The geodesic $\eta_{1}$ converges to $z \in \check{S}$ if and 
only if the stabilizer $\operatorname{Stab}_{G_{\mathcal{D}}}\left(\eta_{1}\right)$ is a parabolic subgroup $P$ which is contained in the maximal parabolic subgroup $Q<G_{\mathcal{D}}$. Then $\sup _{t>0} d_{\mathcal{D}}\left(\eta_{1}(t), \eta_{2}(t)\right)<\infty$ implies that $\operatorname{Stab}_{G_{\mathcal{D}}}\left(\eta_{1}\right)=\operatorname{Stab}_{G_{\mathcal{D}}}\left(\eta_{2}\right)=P$. Now $P<Q$ and $P$ cannot be contained in any other conjugate of $Q$, hence $\lim _{t \rightarrow \infty} \eta_{2}(t)=z$.

We shall as usual say that a geodesic ray $r:[0, \infty) \rightarrow \mathcal{D}$ is of type $P$, where $P$ is a parabolic subgroup of $G_{\mathcal{D}}$, if the stabilizer of the point in $\mathcal{D}(\infty)$ defined by $r$ is $P$ or, what amounts to the same, if

$$
P=\left\{g \in G_{\mathcal{D}}: \sup _{t \geq 0} d_{\mathcal{D}}(g r(t), r(t))<+\infty\right\}
$$

By way of example, we notice that the geodesic

$$
\begin{aligned}
r_{0}:[0, \infty) & \longrightarrow \mathcal{D} \\
t & \longmapsto \sum_{j=1}^{r} \tanh (t) E_{\gamma_{j}}
\end{aligned}
$$

is of type $Q$; this is the geodesic contained in $\Delta_{0}$ connecting 0 to $\sum_{j=1}^{r} \tanh (t) E_{\gamma_{j}}$ in $\overline{\mathcal{D}}$. We should observe here that there are many geodesics connecting 0 to $\sum_{j=1}^{r} E_{\gamma_{j}}$ in $\overline{\mathcal{D}}$, and they need not be at a finite distance from $r_{0}$. A typical example is given by

$$
t \mapsto \sum_{j=1}^{r} \tanh \left(a_{j} t\right) E_{\gamma_{j}}
$$

where $0<a_{1}<\cdots<a_{r}$. However we have the following:

Proposition 3.4. For any $x \in \mathcal{D}$ and $z \in \check{S}_{\mathcal{D}}$, there is a unique diagonal disk $\Delta_{x, z} \subset \mathcal{D}$ with $\{x, z\} \subset \overline{\Delta_{x, z}}$. Moreover, if $r_{x, z}$ denotes the unique geodesic ray in $\Delta_{x, z}$ joining $x$ to $z$, then $r_{x, z}$ is of type $Q_{z}:=\operatorname{Stab}_{G_{\mathcal{D}}}(z)$. Furthermore, for every $x_{1}, x_{2} \in \mathcal{D}$ and $z \in \check{S}_{\mathcal{D}}$, we have that

$$
\sup _{t \geq 0} d_{\mathcal{D}}\left(r_{x_{1}, z}(t), r_{x_{2}, z}(t)\right)<+\infty
$$

Proof. Concerning the existence of such a disk, observe that the diagonal $G_{\mathcal{D}}$-action on $\mathcal{D} \times \check{S}_{\mathcal{D}}$ is transitive; indeed $Q$ acts transitively on $\mathcal{D}$. Thus we may assume that $x=0$ and $z=\sum_{j=1}^{r} \tanh (t) E_{\gamma_{j}}$. But then $\Delta_{0}$ and $\mathrm{r}_{0}$ (see (3.7)) are the objects we sought for.

Let for the moment $r_{x, z}^{\Delta}$ denote the geodesic joining $x$ to $z$ inside $\bar{\Delta}$, where $\Delta$ is a diagonal disk. Let $z \in \check{S}_{\mathcal{D}}$, and consider $x \in \Delta, x^{\prime} \in \Delta^{\prime}$ both diagonal disks with $z \in \partial \Delta \cap \partial \Delta^{\prime}$. Then there is $g \in G_{\mathcal{D}}$ with

$$
g(\Delta)=\Delta^{\prime}, \quad g x=x^{\prime}, \quad g z=z,
$$

that is $g \in Q_{z}$. In particular

$$
g\left(r_{x, z}^{\Delta}\right)=r_{x^{\prime}, z}^{\Delta}
$$

Let $\mathcal{D}=\mathcal{D}_{1} \times \cdots \times \mathcal{D}_{n}$ be a decomposition into irreducible components and, accordingly, $G_{\mathcal{D}}=G_{\mathcal{D}_{1}} \times \cdots \times G_{\mathcal{D}_{n}}, x=\left(x_{1}, \ldots, x_{n}\right), x^{\prime}=\left(x_{1}^{\prime}, \ldots, x_{n}^{\prime}\right)$ and $z=\left(z_{1}, \ldots, z_{n}\right)$. Now if $p_{i}: \mathcal{D} \rightarrow \mathcal{D}_{i}$ denotes the projection on to the $i$-th factor, we observe that $p_{i}\left(r_{x, z}^{\Delta}\right)$ is a ray with parametrization proportional to the arclength and of type $Q_{z_{i}}$. 
Since now $Q_{z_{i}}$ is maximal parabolic, there is a unique geodesic ray $r_{i}^{x_{i}}:[0, \infty) \rightarrow \mathcal{D}_{i}$ starting at $x_{i}$ of type $Q_{z_{i}}$ and hence

for some $a_{i}>0$. Similarly,

$$
r_{x, z}^{\Delta}(t)=\left(r_{i}^{x_{i}}\left(a_{i} t\right)\right)
$$

$$
r_{x^{\prime}, z}^{\Delta^{\prime}}(t)=\left(r_{i}^{x_{i}^{\prime}}\left(b_{i} t\right)\right)
$$

for some $b_{i}>0$. If now $g=\left(g_{1}, \ldots, g_{n}\right)$, according to (3.8) we have

$$
g_{i}\left(r_{i}^{x_{i}}\left(a_{i} t\right)\right)=r_{i}^{x_{i}^{\prime}}\left(b_{i} t\right), \text { for all } t \geq 0,
$$

which implies, since $g$ is an isometry, that $a_{i}=b_{i}$ for $1 \leq i \leq n$. Finally, since $g_{i} \in Q_{z_{i}}$, we have that

and hence

$$
\sup _{s \geq 0} d_{\mathcal{D}_{i}}\left(r_{i}^{x_{i}}(s), r_{i}^{x_{i}^{\prime}}(s)\right)<+\infty
$$

$$
\sup _{t \geq 0} d_{\mathcal{D}}\left(r_{x, z}^{\Delta}(t), r_{x^{\prime}, z}^{\Delta \Delta^{\prime}}(t)\right)<+\infty .
$$

It remains to show the uniqueness assertion. For that, let $\Delta, \Delta^{\prime}$ be diagonal disks with $x \in \Delta \cap \Delta^{\prime}$ and $z \in \partial \Delta \cap \partial \Delta^{\prime}$. Because of (3.9) we have that

$$
r_{x, z}^{\Delta}(t)=r_{x^{\prime}, z}^{\Delta^{\prime}}(t)
$$

for all $t \geq 0$. Thus the holomorphic disks $\Delta, \Delta^{\prime}$ contain a half line in common and hence coincide.

3.2 The Bergmann cocycle and maximal triples. Let us denote by $\overline{\mathcal{D}}$ the closure of $\mathcal{D}$ in $\mathfrak{p}_{+}$.

Definition 3.5. We define

$$
\overline{\mathcal{D}}^{(2)}:=\left\{(z, w) \in \overline{\mathcal{D}} \times \overline{\mathcal{D}}: h_{\mathcal{D}}(z, w) \neq 0\right\}
$$

$\overline{\mathcal{D}}^{[2]}:=\left\{(z, w) \in \overline{\mathcal{D}}^{2}\right.$ : there exists some geodesic in $\mathcal{D}$ connecting $z$ to $\left.w\right\}$.

Then $\overline{\mathcal{D}}^{(2)}$ is a star-shaped domain and is the maximal subset of $\overline{\mathcal{D}}^{2}$ to which the Bergmann kernel $k_{\mathcal{D}}$ extends continuously. Moreover, expanding [C $\varnothing$, Prop. 4.1] to the nonirreducible case, we have that

$$
\overline{\mathcal{D}}^{[2]} \subset \overline{\mathcal{D}}^{2}
$$

If

$$
\arg k_{\mathcal{D}}: \overline{\mathcal{D}}^{(2)} \longrightarrow \mathbb{R}
$$

is the continuous determination of the argument of $k_{\mathcal{D}}$ vanishing on the diagonal of $\mathcal{D}^{2}$, then the integral $\int_{\Delta \subset \mathcal{X}} \omega_{\mathcal{X}}$ can be expressed in terms of the function $\arg k_{\mathcal{D}}$. Proposition $3.6[\mathrm{C} \varnothing]$. Let $\Delta(x, y, z) \subset \mathcal{D}$ be a smooth oriented triangle with geodesic sides and vertices $x, y, z \in \mathcal{D}$. Then

$$
\int_{\Delta(x, y, z)} \omega_{\mathcal{D}}=-\left[\arg k_{\mathcal{D}}(x, y)+\arg k_{\mathcal{D}}(y, z)+\arg k_{\mathcal{D}}(z, x)\right] .
$$

Define the following subsets of $\overline{\mathcal{D}}^{3}$ :

$$
\begin{aligned}
& \overline{\mathcal{D}}^{(3)}:=\left\{\left(z_{1}, z_{2}, z_{3}\right) \in \overline{\mathcal{D}}^{3}:\left(z_{i}, z_{j}\right) \in \overline{\mathcal{D}}^{(2)} \text { for all } i \neq j\right\}, \\
& \overline{\mathcal{D}}^{[3]}:=\left\{\left(z_{1}, z_{2}, z_{3}\right) \in \overline{\mathcal{D}}^{3}:\left(z_{i}, z_{j}\right) \in \overline{\mathcal{D}}^{[2]} \text { for all } i \neq j\right\} .
\end{aligned}
$$


Definition 3.7. The Bergmann cocycle

$$
\beta_{\mathcal{D}}: \overline{\mathcal{D}}^{(3)} \rightarrow \mathbb{R}
$$

is defined by

$$
\beta_{\mathcal{D}}(x, y, z):=-\frac{1}{2 \pi}\left[\arg k_{\mathcal{D}}(x, y)+\arg k_{\mathcal{D}}(y, z)+\arg k_{\mathcal{D}}(z, x)\right] .
$$

It is a $G_{\mathcal{D}}$-invariant alternating continuous function which satisfies the cocycle identity $d \beta\left(z_{1}, z_{2}, z_{3}, z_{4}\right)=0$ whenever $\left(z_{i}, z_{j}, z_{k}\right) \in \overline{\mathcal{D}}^{(3)}$ for all $1 \leq i, j, k \leq 4$ pairwise distinct.

In terms of the decomposition $\mathcal{D}=\mathcal{D}_{1} \times \cdots \times \mathcal{D}_{n}$ into irreducible factors, we have the following formulas for the above mentioned objects:

$$
\begin{aligned}
& h_{\mathcal{D}}\left(\left(z_{1}, \ldots, z_{n}\right),\left(w_{1}, \ldots, w_{n}\right)\right)=\prod_{i=1}^{n} h_{\mathcal{D}_{i}}\left(z_{i}, w_{i}\right), \\
& \overline{\mathcal{D}}^{(2)}=\left\{(z, w) \in \overline{\mathcal{D}}^{2}:\left(z_{i}, w_{i}\right) \in \overline{\mathcal{D}}_{i}^{(2)}, 1 \leq i \leq n\right\}, \\
& \overline{\mathcal{D}}^{[2]}=\left\{(z, w) \in \overline{\mathcal{D}}^{2}:\left(z_{i}, w_{i}\right) \in \overline{\mathcal{D}}_{i}^{[2]}, 1 \leq i \leq n\right\},
\end{aligned}
$$

analogous formulas for $\overline{\mathcal{D}}^{(3)}$ and $\overline{\mathcal{D}}^{[3]}$,

$$
\beta_{\mathcal{D}}(x, y, z)=\sum_{i=1}^{n} \beta_{\mathcal{D}_{i}}\left(x_{i}, y_{i}, z_{i}\right) \text { whenever }(x, y, z) \in \overline{\mathcal{D}}^{(3)} \text {. }
$$

From (3.14) and Theorem 2.8 we deduce that $\left|\beta_{\mathcal{D}}\right| \leq \mathrm{r}_{\mathcal{D}} / 2$ and clearly

$$
\beta_{\mathcal{D}}(x, y, z)=\frac{\mathrm{r}_{\mathcal{D}}}{2} \text { if and only if } \beta_{\mathcal{D}_{i}}\left(x_{i}, y_{i}, z_{i}\right)=\frac{\mathrm{r}_{\mathcal{D}_{i}}}{2} \text { for all } 1 \leq i \leq n .
$$

Theorem 3.8. Suppose that $(x, y, z) \in \overline{\mathcal{D}}^{[3]}$ is such that $\beta_{\mathcal{D}}(x, y, z)=\mathrm{r}_{\mathcal{D}} / 2$, then

(1) The points $x, y, z$ lie on the Shilov boundary $\check{S}_{\mathcal{D}}$; and

(2) there exists a unique diagonal disk $d: \overline{\mathbb{D}} \rightarrow \overline{\mathcal{D}}$ such that $d(1)=x, d(i)=y$, $d(-1)=z$. Moreover,

$$
d(\mathbb{D}) \subset\left\{p \in \mathcal{D}: p \text { is fixed by } \operatorname{Stab}_{G_{\mathcal{D}}}(x, y, z)\right\}
$$

with equality if $\mathcal{D}$ is irreducible.

(3) The group $G_{\mathcal{D}}$ acts transitively on the set

of maximal triples.

$$
\left\{(x, y, z) \in \overline{\mathcal{D}}^{[3]}: \beta_{\mathcal{D}}(x, y, z)=\frac{\mathrm{r}_{\mathcal{D}}}{2}\right\}
$$

Proof. All the above assertions are due to Clerc and Ørsted in the irreducible case, $[\mathrm{CØ]}$. In the general case, the first assertion follows from (3.15) and Lemma 3.2(1).

In the second assertion, only the uniqueness needs to be verified, but this follows easily from the fact that a totally geodesic map $\mathbb{D} \rightarrow \mathbb{D}$ is necessarily isometric.

The last assertion follows immediately from (3.15), (3.13) and the irreducible case. 
3.3 On subdomains of maximal rank. The main goal of this section is to show that if $\mathcal{D}^{\prime}, \mathcal{D}^{\prime \prime}$ are subdomains of $\mathcal{D}$ of maximal rank, that is $\mathrm{r}_{\mathcal{D}}=\mathrm{r}_{\mathcal{D}^{\prime}}=\mathrm{r}_{\mathcal{D}^{\prime \prime}}$, whose Shilov boundaries coincide, then $\mathcal{D}^{\prime}=\mathcal{D}^{\prime \prime}$. We begin with the following.

Lemma 3.9. Let $\mathcal{D}^{\prime} \subset \mathcal{D}$ be a subdomain of maximal rank. Then,

(1) $k_{\mathcal{D}^{\prime}}=\left.k_{\mathcal{D}}\right|_{\mathcal{D}^{\prime}}$

(2) $\overline{\overline{\mathcal{D}}^{\prime}}[3]=\left(\overline{\mathcal{D}^{\prime}}\right)^{3} \cap \overline{\mathcal{D}}^{[3]}$;

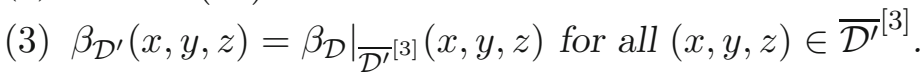

Proof. (1) We observed already that the normalized Bergmann metric on $\mathcal{D}^{\prime}$ is the restriction of the normalized Bergmann metric on $\mathcal{D}$ (see Corollary 2.20), thus the first assertion follows readily.

(2) The second assertion is obvious.

(3) To see the third assertion, observe first of all that if $(x, y, z) \in \mathcal{D}^{\prime 3}$, then the equality follows from (1). Furthermore, continuity of the Bergmann cocycle and the fact that $\mathcal{D}^{\prime 3}$ is dense in $\overline{\mathcal{D}^{\prime}}$ complete the proof.

Lemma 3.10. Let $\mathcal{D}^{\prime} \subset \mathcal{D}$ be a subdomain of maximal rank, $A_{\mathcal{D}^{\prime}}$ the associated subgroup of Hermitian type and $f: \mathbb{D} \rightarrow \mathcal{D}^{\prime}$ a diagonal embedding. Then

$$
\mathcal{N}_{G_{\mathcal{D}}}\left(\check{S}_{\mathcal{D}^{\prime}}\right)=\mathcal{N}_{G_{\mathcal{D}}}\left(\mathcal{D}^{\prime}\right) \subset A_{\mathcal{D}^{\prime}} \cdot \mathcal{Z}_{G_{\mathcal{D}}}(f)
$$

where $\mathcal{Z}_{G_{\mathcal{D}}}(f)=\left\{g \in G_{\mathcal{D}}: g x=x\right.$ for all $\left.x \in f(\mathbb{D})\right\}$.

Proof. Let $x:=f(1), y:=f(i)$ and $z:=f(-1)$, and pick $g \in \mathcal{N}_{G_{\mathcal{D}}}\left(\check{S}_{\mathcal{D}^{\prime}}\right)$. We have that since $(x, y, z) \in \check{S}_{\mathcal{D}^{\prime}}^{[3]}$ then $(g x, g y, g z) \in \check{S}_{\mathcal{D}^{\prime}}^{[3]}$ and, using Lemma $3.9(3)$,

$$
\frac{\mathrm{r}_{\mathcal{D}}}{2}=\beta_{\mathcal{D}^{\prime}}(x, y, z)=\beta_{\mathcal{D}}(x, y, z)=\beta_{\mathcal{D}}(g x, g y, g z)=\beta_{\mathcal{D}^{\prime}}(g x, g y, g z),
$$

which implies by Theorem 3.8(3) that there exists $h \in A_{\mathcal{D}^{\prime}}$ with $h x=g x, h y=g y$, $h z=g z$ and thus $g \in A_{\mathcal{D}^{\prime}} \cdot \operatorname{Stab}_{G_{\mathcal{D}}}(x, y, z)$. Since $\operatorname{Stab}_{G_{\mathcal{D}}}(x, y, z) \subset \mathcal{Z}_{G_{\mathcal{D}}}(f)$ (see Theorem 3.8(2)), we obtain that $\mathcal{N}_{G_{\mathcal{D}}}\left(\check{S}_{\mathcal{D}^{\prime}}\right) \subset A_{\mathcal{D}^{\prime}} \cdot \mathcal{Z}_{G_{\mathcal{D}}}(f)$.

Let now $p \in f(\mathbb{D}) \subset \mathcal{D}^{\prime}$. Then $\mathcal{N}_{G_{\mathcal{D}}}\left(\check{S}_{\mathcal{D}^{\prime}}\right) \cdot p \subset A_{\mathcal{D}^{\prime}}(p)=\mathcal{D}^{\prime}$, and since $\mathcal{N}_{G_{\mathcal{D}}}\left(\check{S}_{\mathcal{D}^{\prime}}\right)=\mathcal{N}_{G_{\mathcal{D}}}\left(\check{S}_{\mathcal{D}^{\prime}}\right) \cdot A_{\mathcal{D}^{\prime}}$, we obtain that $\mathcal{N}_{G_{\mathcal{D}}}\left(\check{S}_{\mathcal{D}^{\prime}}\right) \mathcal{D}^{\prime}=\mathcal{D}^{\prime}$ and hence $\mathcal{N}_{G_{\mathcal{D}}}\left(\check{S}_{\mathcal{D}^{\prime}}\right) \subset \mathcal{N}_{G_{\mathcal{D}}}\left(\mathcal{D}^{\prime}\right)$. The opposite inclusion is clear.

Proposition 3.11. Let $\mathcal{D}^{\prime}, \mathcal{D}^{\prime \prime}$ be subdomains of $\mathcal{D}$ of maximal rank, and assume that $\check{S}_{\mathcal{D}^{\prime}}=\check{S}_{\mathcal{D}^{\prime \prime}}$. Then $\mathcal{D}^{\prime}=\mathcal{D}^{\prime \prime}$.

Proof. Let $x, y, z \in \check{S}_{\mathcal{D}^{\prime}}=\check{S}_{\mathcal{D}^{\prime \prime}}$ with $\beta_{\mathcal{D}}(x, y, z)=\mathrm{r}_{\mathcal{D}} / 2$. Then

$$
\beta_{\mathcal{D}^{\prime}}(x, y, z)=\frac{\mathrm{r}_{\mathcal{D}^{\prime}}}{2} \quad \text { and } \quad \beta_{\mathcal{D}^{\prime \prime}}(x, y, z)=\frac{\mathrm{r}_{\mathcal{D}^{\prime \prime}}}{2},
$$

and there are diagonal disks

$$
f_{\mathcal{D}^{\prime}}: \overline{\mathbb{D}} \rightarrow \overline{\mathcal{D}^{\prime}} \quad \text { and } \quad f_{\mathcal{D}^{\prime \prime}}: \overline{\mathbb{D}} \rightarrow \overline{\mathcal{D}^{\prime \prime}}
$$

with

$$
\begin{aligned}
f_{\mathcal{D}^{\prime}}(1) & =x=f_{\mathcal{D}^{\prime \prime}}(1) \\
f_{\mathcal{D}^{\prime}}(i) & =y=f_{\mathcal{D}^{\prime \prime}}(i) \\
f_{\mathcal{D}^{\prime}}(-1) & =z=f_{\mathcal{D}^{\prime \prime}}(-1) .
\end{aligned}
$$


Those are also diagonal disks in $\mathcal{D}$ and hence by uniqueness we have $f_{\mathcal{D}^{\prime}}=f_{\mathcal{D}^{\prime \prime}}$, and in particular $\mathcal{D}^{\prime} \cap \mathcal{D}^{\prime \prime} \neq \emptyset$. Pick now $p \in \mathcal{D}^{\prime} \cap \mathcal{D}^{\prime \prime}$ and apply Lemma 3.10 to obtain

$$
\mathcal{D}^{\prime}=\mathcal{N}_{G_{\mathcal{D}}}\left(\check{S}_{\mathcal{D}^{\prime}}\right) \cdot p=\mathcal{N}_{G_{\mathcal{D}}}\left(\check{S}_{\mathcal{D}^{\prime \prime}}\right) \cdot p=\mathcal{D}^{\prime \prime} \text {. }
$$

\section{Structure Theorem for Tight Embeddings, I}

The main objective in this section is to prove the following structure theorem.

Theorem 4.1. Let $H, G$ be Lie groups of Hermitian type, $\rho: H \rightarrow G$ a continuous tight homomorphism and $f: \mathcal{D}^{\prime} \rightarrow \mathcal{D}$ the corresponding $\rho$-equivariant tight totally geodesic map. Then $f$ extends continuously to a $\rho$-equivariant map

$$
\check{f}: \check{S}_{\mathcal{D}^{\prime}} \rightarrow \check{S}_{\mathcal{D}} \text {. }
$$

Moreover the centralizer $\mathcal{Z}_{G}(\rho(H))$ is compact.

4.1 The case of the Poincaré disk. We will first prove Theorem 4.1 in the case when $\mathcal{D}^{\prime}=\mathbb{D}$ is the Poincaré disk.

Proposition 4.2. Let $L$ be a finite covering of $\mathrm{PU}(1,1)$ and $G$ a group of Hermitian type. Let $\rho: L \rightarrow G$ a continuous tight homomorphism with finite kernel and $f: \mathbb{D} \rightarrow \mathcal{D}$ the corresponding totally geodesic tight embedding. Then,

(1) $f$ extends continuously to a map

$$
\check{f}: \partial \mathbb{D} \rightarrow \partial \mathcal{D},
$$

which is $\rho$-equivariant and has image $\check{f}(\partial \mathbb{D}) \subset \check{S}_{\mathcal{D}}$.

(2) If $x \neq y$ in $\partial \mathbb{D}$, then $\check{f}(x)$ and $\check{f}(y)$ are transverse.

(3) The centralizer $\mathcal{Z}_{G}(\rho(L))$ is compact.

Proof. Observe that since $f$ is equivariant, there exists a constant $c>0$ such that

$$
d_{\mathcal{D}}(f(x), f(y))=c d_{\mathbb{D}}(x, y) .
$$

Next, let $r_{1}, r_{2}: \mathbb{R}^{+} \rightarrow \mathbb{D}$ be geodesic rays representing a given point $\xi \in \partial \mathbb{D}$ and $a \geq 0$ such that $\lim _{t \rightarrow \infty} d_{\mathbb{D}}\left(r_{1}(t), r_{2}(t+a)\right)=0$. Then

$$
\lim _{t \rightarrow \infty} d_{\mathcal{D}}\left(f\left(r_{1}(t)\right), f\left(r_{2}(t+a)\right)\right)=0
$$

and, by Lemma 3.1,

$$
\lim _{t \rightarrow \infty}\left\|f\left(r_{1}(t)\right)-f\left(r_{2}(t+a)\right)\right\|_{\text {eucl }}=0
$$

which shows that the geodesics $t \mapsto f\left(r_{1}(t)\right)$ and $t \mapsto f\left(r_{2}(t)\right)$ have the same endpoints in $\partial \mathcal{D}$. This produces a well-defined and equivariant (continuous) extension $\check{f}: \partial \mathbb{D} \rightarrow \partial \mathcal{D}$ of $f$. Observe that for all $x \neq y$ in $\partial \mathbb{D},(\check{f}(x), \check{f}(y)) \in \overline{\mathcal{D}}^{[2]}$ since $x, y$ and thus $\check{f}(x), \check{f}(y)$ are joined by a geodesic.

Now, for the (normalized) Kähler forms $\omega_{\mathcal{D}} \in \Omega^{2}(\mathcal{D})^{G}$ and $\omega_{\mathbb{D}} \in \Omega^{2}(\mathbb{D})^{L}$ we have since $f$ is tight

$$
f^{*}\left(\omega_{\mathcal{D}}\right)=\epsilon \omega_{\mathbb{D}},
$$

where $|\epsilon|=\mathrm{r}_{\mathcal{D}}$. Composing if necessary with an orientation reversing isometry of $\mathbb{D}$ we may assume $\epsilon=\mathrm{r}_{\mathcal{D}}$. 
This implies by integration over simplices with geodesic sides in $\mathbb{D}$ and continuity of $\check{f}$, as well as of $\beta_{\mathcal{D}}$ on $\overline{\mathcal{D}}^{[3]}$ and of $\beta_{\mathbb{D}}$ on $\overline{\mathbb{D}}^{[3]}$ that

$$
\beta(\check{f}(x), \check{f}(y), \check{f}(z))=\mathrm{r}_{\mathcal{D}} \beta_{\mathbb{D}}(x, y, z)
$$

for all $(x, y, z) \in \overline{\mathbb{D}}^{[3]}$.

Applying this to a positively oriented triple $(x, y, z)$ we get

$$
\beta(\check{f}(x), \check{f}(y), \check{f}(z))=\frac{\mathrm{r}_{\mathcal{D}}}{2}
$$

and hence, by Theorem 3.8, $\check{f}(x) \in \check{S}_{\mathcal{D}}$. This shows (1). The second assertion follows from the fact we already remarked that $(\check{f}(x), \check{f}(y)) \in \overline{\mathcal{D}}^{[2]}$ if $x \neq y$. For the third assertion, let $\eta: \mathbb{R}^{+} \rightarrow \mathbb{D}$ be a geodesic ray with $\lim _{t \rightarrow \infty} \eta(t)=x$. Then $f(\eta)$ is a geodesic ray in $\mathcal{D}$ converging to $\check{f}(x)$. For $g \in \mathcal{Z}_{G}(\rho(L))$ the geodesic ray $g \cdot f(\eta)$ is at bounded distance from $f(\eta)$, hence Lemma 3.3 implies that

$$
g \check{f}(x)=\check{f}(x) .
$$

In particular $\mathcal{Z}_{G}(\rho(L)) \subset \operatorname{Stab}_{G}(\check{f}(x), \check{f}(y), \check{f}(z))$ which by Theorem 3.8(2) is compact.

Corollary 4.3. Let $H, G$ be groups of Hermitian type and let $\left\{\kappa_{H, i}^{\mathrm{b}}\right\}_{i=1}^{n} \in \mathrm{H}_{\mathrm{cb}}^{2}(H, \mathbb{R})$ be the basis of $\mathrm{H}_{\mathrm{cb}}^{2}(H, \mathbb{R})$ corresponding to the decomposition $\mathcal{Y}=\mathcal{Y}_{1} \times \cdots \times \mathcal{Y}_{n}$ into irreducible factors of the symmetric space $\mathcal{Y}$ associated to $H$. Let $\rho: H \rightarrow G$ be a tight homomorphism and assume that $\rho_{\mathrm{b}}^{*} \kappa_{G}^{\mathrm{b}}=\sum_{i=1}^{n} \lambda_{i} \kappa_{H, i}^{\mathrm{b}}$. If $H=H_{1} \cdots H_{n}$ is the decomposition of $H$ into connected almost simple groups where $H_{i}$ corresponds to $\mathcal{Y}_{i}$, then $\lambda_{i}=0$ if and only if $H_{i}$ is in the kernel of $\rho$.

Proof. If $f: \mathcal{Y} \rightarrow \mathcal{X}$ is a tight $\rho$-equivariant map, then

$$
f^{*}\left(\omega_{\mathcal{X}}\right)=\sum_{i=1}^{n} \lambda_{i} \omega_{\mathcal{Y}, i}
$$

where $\omega_{\mathcal{Y}, i}=p_{i}^{*}\left(\omega_{\mathcal{Y}_{i}}\right)$. Assume that $\lambda_{i} \neq 0$ for $1 \leq i \leq \ell$ and $\lambda_{\ell+1}=\cdots=$ $\lambda_{n}=0$. For $1 \leq i \leq \ell$ define $t_{i}: \mathbb{D} \rightarrow \mathcal{Y}_{i}$ to be the embedding as a diagonal disk (Definition 2.18) composed with an isometry of $\mathbb{D}$ reversing the orientation in the case in which $\lambda_{i}<0$, and let $\rho_{i}: \operatorname{SU}(1,1) \rightarrow \operatorname{Isom}\left(\mathcal{Y}_{i}\right)^{\circ}$ be the associated homomorphism. Let $b_{i} \in \mathcal{Y}_{i}$ be a basepoint and define

$$
\begin{aligned}
t: \mathbb{D} & \longrightarrow \mathcal{Y}_{1} \times \cdots \times \mathcal{Y}_{n} \\
z & \longmapsto\left(t_{1}(z), \ldots, t_{\ell}(z), b_{\ell+1}, \ldots, b_{n}\right) .
\end{aligned}
$$

and

$$
\begin{aligned}
\pi: \mathrm{SU}(1,1) & \longrightarrow \operatorname{Isom}(\mathcal{Y})^{\circ} \\
g & \longmapsto\left(\rho_{1}(g), \ldots, \rho_{\ell}(g), e, \ldots, e\right) .
\end{aligned}
$$

Taking into account that $H$ is a finite extension of $\operatorname{Isom}(\mathcal{Y})^{\circ}$, let

$$
\widetilde{\pi}: L \rightarrow H
$$

be the lift of $\pi$ to a finite extension $L$ of $\mathrm{SU}(1,1)$. Then

$$
t^{*}\left(\sum_{i=1}^{n} \lambda_{i} \omega_{\mathcal{Y}, i}\right)=\left(\sum_{i=1}^{n}\left|\lambda_{i}\right| \mathrm{r}_{\mathcal{Y}_{i}}\right) \omega_{\mathbb{D}}=\mathrm{r}_{\mathcal{X}} \omega_{\mathbb{D}},
$$


where the last equality follows from the fact that $f$ is tight (Corollary 2.17). Thus

$$
f \circ t: \mathbb{D} \longrightarrow \mathcal{X}
$$

is tight and equivariant with respect to the homomorphism

$$
\rho \circ \tilde{\pi}: L \longrightarrow G .
$$

Let now $H=H_{1} \cdots H_{n}$ be the decomposition of $H$ into connected almost simple groups, where $H_{i}$ is a finite extension of $\operatorname{Isom}\left(\mathcal{Y}_{i}\right)^{\circ}$. In particular, for $\ell+1 \leq j \leq n$, $H_{j}$ commutes with $\widetilde{\pi}(L)$ and hence $\rho\left(H_{j}\right)$ commutes with $(\rho \circ \tilde{\pi})(L)$ which implies that, for $\ell+1 \leq j \leq n, \rho\left(H_{j}\right)$ is contained in $\mathcal{Z}_{G}(\rho \widetilde{\pi}(L))$ which is compact by virtue of Proposition 4.2, and hence $\rho\left(H_{j}\right)=e$.

The converse, namely that $\rho\left(H_{j}\right)=e$ implies that $\lambda_{j}=0$ is clear.

4.2 Positivity. Let $G$ be a group of type $(\mathrm{RH})$. We shall use freely the notation from section 2.3. In this section, we prove that the notion of tightness does not depend on the choice of the specific Kähler class $\kappa_{G}^{\mathrm{b}} \in \mathrm{H}_{\mathrm{cb}}^{2}(G, \mathbb{R})$ which we used to define it but, indeed, it depends only on the choice of a $G_{\mathcal{X}}$-invariant complex structure on $\mathcal{X}$. In the case when $\mathcal{X}$ is irreducible this is immediate from $\mathrm{H}_{\mathrm{cb}}^{2}(G, \mathbb{R})=\mathbb{R} \kappa_{G}^{\mathrm{b}}$. In the general case, however, one could have some "cancellations" coming from different factors, but we are going to set up conditions which will allow us some freedom to choose the Kähler classes according to the context.

Again, let $\mathcal{X}=\mathcal{X}_{1} \times \cdots \times \mathcal{X}_{n}$ be the decomposition into irreducible factors. Then any choice of $G_{\mathcal{X}}$-invariant complex structure $\mathcal{J}_{\mathcal{X}}$ determines a $G_{\mathcal{X}_{i}}$-invariant complex structure $\mathcal{J}_{\mathcal{X}_{i}}$ on $\mathcal{X}_{i}$ and hence an orientation on $\mathrm{H}_{\mathrm{cb}}^{2}\left(G_{\mathcal{X}_{i}}, \mathbb{R}\right)$. Conversely, any choice of orientation on each $\mathrm{H}_{\mathrm{cb}}^{2}\left(G_{\mathcal{X}_{i}}, \mathbb{R}\right)$ determines a complex structure on $\mathcal{X}$.

Definition 4.4. A bounded cohomology class $\alpha \in \mathrm{H}_{\mathrm{cb}}^{2}(G, \mathbb{R})$ is positive if

$$
\alpha=\sum_{i=1}^{n} \mu_{i} \kappa_{G, i}^{\mathrm{b}}
$$

with $\mu_{i} \geq 0$ for all $i=1, \cdots, n$ and strictly positive if the $\mu_{i}>0$, for all $i=1, \ldots, n$.

The cone of positive Kähler classes in $\mathrm{H}_{\mathrm{cb}}^{2}(G, \mathbb{R})$ is denoted by $\mathrm{H}_{\mathrm{cb}}^{2}(G, \mathbb{R}) \geq 0$ and the cone of strictly positive Kähler classes by $\mathrm{H}_{\mathrm{cb}}^{2}(G, \mathbb{R})^{>0}$.

Note that the cone $\mathrm{H}_{\mathrm{cb}}^{2}(G, \mathbb{R})^{\geq 0}$ depends only on the complex structure $\mathcal{J}$. In fact $\mathrm{H}_{\mathrm{cb}}^{2}(G, \mathbb{R})^{>0}$ coincides with the set of bounded Kähler classes associated to any $G$-invariant Hermitian metric on $\mathcal{X}$ compatible with the complex structure $\mathcal{J}$; in particular we have that $\kappa_{G}^{\mathrm{b}} \in \mathrm{H}_{\mathrm{cb}}^{2}(G, \mathbb{R})^{>0}$.

Proposition 4.5. Let $\rho: H \rightarrow G$ be a homomorphism of a locally compact group $H$ into a group $G$ of type $(R H)$. Then the following are equivalent:

(1) $\rho$ is tight;

(2) $\rho$ is $\alpha$-tight for some $\alpha \in \mathrm{H}_{\mathrm{cb}}^{2}(G, \mathbb{R})^{>0}$;

(3) $\rho$ is $\alpha$-tight for all $\alpha \in \mathrm{H}_{\mathrm{cb}}^{2}(G, \mathbb{R})^{>0}$;

(4) $\rho$ is $\alpha$-tight for all $\alpha \in \mathrm{H}_{\mathrm{cb}}^{2}(G, \mathbb{R}) \geq 0$.

This is a consequence of the special Banach space structure of $\mathrm{H}_{\mathrm{cb}}^{2}$. 
Lemma 4.6. Let $V$ be a Banach space. Let $v_{i} \in V, i=1, \ldots, k$, be vectors such that

$$
\left\|\sum_{i=1}^{n} v_{i}\right\|=\sum_{i=1}^{n}\left\|v_{i}\right\|
$$

Then for every real numbers $\mu_{1}, \ldots, \mu_{n} \geq 0$, we have that

$$
\left\|\sum_{i=1}^{n} \mu_{i} v_{i}\right\|=\sum_{i=1}^{n} \mu_{i}\left\|v_{i}\right\| .
$$

Proof. By virtue of the Hahn-Banach theorem the norm of a vector $w \in V$ is given by

$$
\|w\|=\sup \{|\lambda(w)|: \lambda: V \rightarrow \mathbb{R} \text { is a linear form of norm } 1\} .
$$

By hypothesis, if we fix $\epsilon>0$, there exists $\lambda: V \rightarrow \mathbb{R}$ a linear form of norm 1 such that

$$
\lambda\left(\sum_{i=1}^{n} v_{i}\right)>\left\|\sum_{i=1}^{n} v_{i}\right\|-\epsilon=\left(\sum_{i=1}^{n}\left\|v_{i}\right\|\right)-\epsilon .
$$

From this and the fact that $\lambda\left(v_{i}\right) \leq\left\|v_{i}\right\|$, we must have that

$$
\lambda\left(v_{i}\right) \geq\left\|v_{i}\right\|-\epsilon
$$

for all $i=1, \ldots, n$, and hence, if $\mu_{i} \geq 0$,

$$
\mu_{i} \lambda\left(v_{i}\right) \geq \mu_{i}\left\|v_{i}\right\|-\mu_{i} \epsilon .
$$

But then

$$
\lambda\left(\sum_{i=1}^{n} \mu_{i} v_{i}\right) \geq\left(\sum_{i=1}^{n} \mu_{i}\left\|v_{i}\right\|\right)-\epsilon \sum_{i=1}^{n} \mu_{i},
$$

which, since $\epsilon$ is arbitrary, shows the assertion.

Proof of Proposition 4.5. We start by showing that $(1) \Rightarrow(4)$. We first verify that the vectors $v_{i}:=\rho_{\mathrm{b}}^{*}\left(\kappa_{G, i}^{\mathrm{b}}\right)$ satisfy the hypotheses of Lemma 4.6 . We have that

$$
\left\|\sum_{i=1}^{n} v_{i}\right\|=\left\|\sum_{i=1}^{n} \rho_{\mathrm{b}}^{*} \kappa_{G, i}^{\mathrm{b}}\right\|=\left\|\rho_{\mathrm{b}}^{*} \kappa_{G}^{\mathrm{b}}\right\|=\left\|\kappa_{G}^{\mathrm{b}}\right\|,
$$

where the last equality follows from the fact that $\rho$ is tight. Moreover, Lemma 2.6(4) implies that

and hence

$$
\left\|v_{i}\right\|=\left\|\rho_{\mathrm{b}}^{*}\left(\kappa_{G, i}^{\mathrm{b}}\right)\right\|=\left\|\kappa_{G, i}^{\mathrm{b}}\right\|
$$

$$
\sum_{i=1}^{n}\left\|v_{i}\right\|=\sum_{i=1}^{n}\left\|\kappa_{G, i}^{\mathrm{b}}\right\|=\left\|\kappa_{G}^{\mathrm{b}}\right\| .
$$

Thus, $\left\|\sum_{i=1}^{n} v_{i}\right\|=\sum_{i=1}^{n}\left\|v_{i}\right\|$, and applying Lemma 4.6 we get

Thus $\rho$ is $\alpha$-tight.

$$
\begin{aligned}
\left\|\rho_{\mathrm{b}}^{*} \alpha\right\| & =\left\|\sum_{i=1}^{n} \mu_{i} \rho_{\mathrm{b}}^{*} \kappa_{G, i}^{\mathrm{b}}\right\|=\sum_{i=1}^{n}\left\|\mu_{i} \rho_{\mathrm{b}}^{*} \kappa_{G, i}^{\mathrm{b}}\right\| \\
& =\sum_{i=1}^{n} \mu_{i}\left\|\kappa_{G, i}^{\mathrm{b}}\right\|=\left\|\sum_{i=1}^{n} \mu_{i} \kappa_{G, i}^{\mathrm{b}}\right\|=\|\alpha\| .
\end{aligned}
$$


The implications $(4) \Rightarrow(3) \Rightarrow(2)$ are obvious.

Finally, to see that $(2) \Rightarrow(1)$, let $\alpha=\sum_{i=1}^{n} \lambda_{i} \kappa_{G, i}^{\mathrm{b}}$ be strictly positive. Then setting $v_{i}:=\lambda_{i} \kappa_{G, i}^{\mathrm{b}}$ and $\mu_{i}:=1 / \lambda_{i}$, the argument above implies that if $\rho$ is $\alpha$-tight then it is $\kappa_{G}^{\mathrm{b}}$-tight.

Definition 4.7. A homomorphism $\rho: H \rightarrow G$ of groups of type (RH) is said to be positive if $\rho_{\mathrm{b}}^{*} \kappa_{G}^{\mathrm{b}} \in \mathrm{H}_{\mathrm{cb}}^{2}(H, \mathbb{R})^{\geq 0}$ and strictly positive if $\rho_{\mathrm{b}}^{*} \kappa_{G}^{\mathrm{b}} \in \mathrm{H}_{\mathrm{cb}}^{2}(H, \mathbb{R})^{>0}$.

The point of the next lemma is to provide a converse to Lemma 2.6(4), for which we need the hypothesis of positivity. Remark that it will be essential that, with the norm on the continuous bounded cohomology, we have that if $v, w$ are positive classes then $\|v+w\|=\|v\|+\|w\|$.

Lemma 4.8. Let $H, G$ be of type $(R H)$ and let $\rho: H \rightarrow G$ be a continuous homomorphism. With the notation in section 2.3, if $\rho_{i}:=q \circ \rho: H \rightarrow G_{\mathcal{X}_{i}}$ is tight and positive for all $i=1, \ldots, n$ then $\rho$ is tight and positive.

Proof. Since we have

$$
\kappa_{G}^{\mathrm{b}}=\sum_{i=1}^{n} \kappa_{G, i}^{\mathrm{b}} \in \mathrm{H}_{\mathrm{cb}}^{2}(G, \mathbb{R})
$$

then

$$
\rho_{\mathrm{b}}^{*} \kappa_{G}^{\mathrm{b}}=\sum_{i=1}^{n}\left(\rho_{i}\right)_{\mathrm{b}}^{*} \kappa_{\mathcal{X}_{i}}^{\mathrm{b}}
$$

Since $\left(\rho_{i}\right)_{\mathrm{b}}^{*} \kappa_{\mathcal{X}_{i}}^{\mathrm{b}}$ are positive for all $i=1, \ldots, n, \rho_{\mathrm{b}}^{*} \kappa_{G}^{\mathrm{b}}$ is positive; this, and the hypothesis that

$$
\left\|\left(\rho_{i}\right)_{\mathrm{b}}^{*} \kappa_{\mathcal{X}_{i}}^{\mathrm{b}}\right\|=\left\|\kappa_{\mathcal{X}_{i}}^{\mathrm{b}}\right\|
$$

allow us to deduce that

$$
\left\|\rho_{\mathrm{b}}^{*} \kappa_{G}^{\mathrm{b}}\right\|=\left\|\sum_{i=1}^{n}\left(\rho_{i}\right)_{\mathrm{b}}^{*} \kappa_{\mathcal{X}_{i}}^{\mathrm{b}}\right\|=\sum_{i=1}^{n}\left\|\left(\rho_{i}\right)_{\mathrm{b}}^{*} \kappa_{\mathcal{X}_{i}}^{\mathrm{b}}\right\|=\sum_{i=1}^{n}\left\|\kappa_{\mathcal{X}_{i}}^{\mathrm{b}}\right\|=\left\|\kappa_{\mathcal{X}}^{\mathrm{b}}\right\|=\left\|\kappa_{G}^{\mathrm{b}}\right\| .
$$

Lemma 4.9. Let $H, G$ be Lie groups of type $(R H), L$ a locally compact group, $\rho: L \rightarrow H$ a tight homomorphism, and $\psi: H \rightarrow G$ a positive tight homomorphism. Then $\psi \circ \rho: L \rightarrow G$ is a tight homomorphism.

Proof. If $\psi$ is positive, $\psi_{\mathrm{b}}^{*} \kappa_{G}^{\mathrm{b}} \in \mathrm{H}_{\mathrm{cb}}^{2}(H, \mathbb{R})^{\geq 0}$. By Proposition 4.5 , if the homomorphism $\rho$ is tight, it is also $\psi_{\mathrm{b}}^{*}\left(\kappa_{G}^{\mathrm{b}}\right)$-tight and Lemma 2.5 concludes the proof.

Lemma 4.10. Let $H, G$ be Lie groups of Hermitian type with associated symmetric spaces $\mathcal{Y}$ and $\mathcal{X}$ with complex structures $\mathcal{J}_{\mathcal{Y}}$ and $\mathcal{J}_{\mathcal{X}}$. Suppose that $\rho: H \rightarrow G$ is a tight homomorphism and $f: \mathcal{Y} \rightarrow \mathcal{X}$ is the corresponding tight map. Then there exists a complex structure $\mathcal{J}^{\prime}$ on $\mathcal{Y}$ such that $\rho$ is tight and positive with respect to $\mathcal{J}^{\prime}$. If moreover $\operatorname{ker} \rho$ is finite, then this structure is unique.

Proof. Since $\rho$ is tight, we have $0 \neq \rho_{\mathrm{b}}^{*} \kappa_{G}^{\mathrm{b}} \in \mathrm{H}_{\mathrm{cb}}^{2}(H, \mathbb{R})$. So, if $\mathcal{Y}$ is irreducible, then $\rho: H \rightarrow G$ is either positive with respect to $\mathcal{J}_{\mathcal{Y}}$ or with respect to $-\mathcal{J}_{\mathcal{Y}}$. 
In the case when $\mathcal{Y}$ is not irreducible, let $\mathcal{Y}=\mathcal{Y}_{1} \times \cdots \times \mathcal{Y}_{n}$ be the decomposition into irreducible factors, and $\mathcal{J}_{i}$ the complex structure on $\mathcal{Y}_{i}$ induced by $\mathcal{J} \mathcal{Y}$. We have

$$
\rho_{\mathrm{b}}^{*} \kappa_{G}^{\mathrm{b}}=\sum_{i=1}^{n} \mu_{i} \kappa_{H, i}^{\mathrm{b}} .
$$

Set $\mathcal{J}_{i}^{\prime}=\epsilon_{i} \mathcal{J}_{i}$, where $\epsilon_{i}=\operatorname{sign}\left(\mu_{i}\right)$ and let $\mathcal{J}^{\prime}$ be the complex structure on $\mathcal{Y}$ which induces the complex structure $\mathcal{J}_{i}^{\prime}$ on $\mathcal{Y}_{i}$. Let $\kappa_{H, i}^{\prime \mathrm{b}}=\operatorname{sign}\left(\mu_{i}\right) \kappa_{H, i}^{\mathrm{b}} \in \mathrm{H}_{\mathrm{cb}}^{2}(H, \mathbb{R})$ be the basis vectors of $\mathrm{H}_{\mathrm{cb}}^{2}(H, \mathbb{R})$ corresponding to $\mathcal{J}^{\prime}$. Then

$$
\rho_{\mathrm{b}}^{*} \kappa_{G}^{\mathrm{b}}=\sum_{i=1}^{n} \operatorname{sign}\left(\mu_{i}\right) \mu_{i} \kappa_{H, i}^{\mathrm{b}},
$$

so $\rho$ is positive with respect to $\mathcal{J}^{\prime}$ and tight. In case $\operatorname{ker} \rho$ is finite we have that $\mu_{i} \neq 0$ for all $1 \leq i \leq n$ (Corollary 4.3) and hence $\mathcal{J}^{\prime}$ is unique.

Proof of Theorem 4.1. Let $\mathcal{D}^{\prime}$ and $\mathcal{D}$ be the bounded domain realizations respectively of $\mathcal{Y}$ and $\mathcal{X}$. Let $f: \mathcal{D}^{\prime} \rightarrow \mathcal{D}$ be a $\rho$-equivariant totally geodesic tight map. Because of Lemma 4.10 we may assume that $f$ is positive. For every $x \in \mathcal{D}^{\prime}$ and $z \in \check{S}_{\mathcal{D}^{\prime}}$, let $\Delta_{x, z} \subset \mathcal{D}^{\prime}$ be the unique diagonal disk given by Proposition 3.4 and

$$
d_{x, z}: \overline{\mathbb{D}} \rightarrow \overline{\Delta_{x, z}}
$$

the unique totally geodesic map with

$$
d_{x, z}(0)=x \quad \text { and } \quad d_{x, z}(1)=z .
$$

Then $f \circ d_{x, z}: \mathbb{D} \rightarrow \mathcal{D}$ is tight (Lemma 4.9) and hence, by Proposition 4.2 extends to

$$
\overline{f \circ d_{x, z}}: \partial \mathbb{D} \rightarrow \check{S}_{\mathcal{D}}
$$

We set

$$
\check{f}_{x}(z):=\left(\overline{f \circ d_{x, z}}\right)(1)=\lim _{t \rightarrow \infty} f\left(r_{x, z}(t)\right) .
$$

If now $x^{\prime}$ is another point in $\mathcal{D}$, we have that

$$
\sup _{t \geq 0} d_{\mathcal{D}^{\prime}}\left(r_{x, z}(t), r_{x^{\prime}, z}(t)\right)<+\infty
$$

and, since $f$ is totally geodesic, also

$$
\sup _{t \geq 0} d_{\mathcal{D}}\left(f\left(r_{x, z}(t)\right), f\left(r_{x^{\prime}, z}(t)\right)\right)<+\infty .
$$

Since $\check{f}_{x}(z) \in \check{S}_{\mathcal{D}}$, we deduce, by $(3.8)$, that $\check{f}_{x^{\prime}}(z)=\check{f}_{x}(z)$; thus the extension $\check{f}: \check{S}_{\mathcal{D}^{\prime}} \rightarrow \check{S}_{\mathcal{D}}$ is independent of $x$ and hence $\rho$-equivariant.

\section{Tight Embeddings and Tube-Type Domains}

Let $\mathcal{X}$ be a Hermitian symmetric space and $\mathcal{D}$ its bounded symmetric domain realization. We will use the concepts and notation from section 3 . 
The real vectors $X_{\gamma_{j}} \in \mathfrak{p}$ associated to the strongly orthogonal roots $\gamma_{j} \in \Lambda$, $j=1, \ldots, r$ (see section 3.1) give rise to the Cayley element

$$
c=\exp \left(\frac{\pi}{4} i \sum_{j=1}^{r} X_{\gamma_{j}}\right) \in G_{\mathbb{C}}=\exp \left(\mathfrak{g}_{\mathbb{C}}\right) .
$$

Remark 5.1. The Cayley element defines the Cayley transformation $\mathfrak{p}_{+} \supset \mathcal{D} \rightarrow$ $\mathcal{H} \subset \mathfrak{p}_{+}$, which sends $\mathcal{D}$ to a Siegel domain $\mathcal{H}$, which, if $\mathcal{X}$ is of tube type (see Definition 2.18), is a tube domain of the form $V \oplus i \Omega$.

The automorphism $\operatorname{Ad}(c)$ of $\mathfrak{g}_{\mathbb{C}}$ is of order 4 if $\mathcal{X}$ is of tube type and of order 8 if $\mathcal{X}$ is not of tube type. When $\mathcal{X}$ is not of tube type $\operatorname{Ad}(c)^{4}$ is an involution of $\mathfrak{g}_{\mathbb{C}}$ which preserves $\mathfrak{g}$ and commutes with the Cartan involution of $\mathfrak{g}=\mathfrak{k} \oplus \mathfrak{p}$ (see e.g. [KW, Th. 4.9]).

We denote by $\mathfrak{g}_{T} \subset \mathfrak{g}$ the fix points of $\operatorname{Ad}(c)^{4}$ in $\mathfrak{g}$ and let $\mathfrak{g}_{T}=\mathfrak{k}_{T} \oplus \mathfrak{p}_{T}$ be its Cartan decomposition. Then the corresponding Hermitian symmetric space $\mathcal{X}_{T}$ is of tube type. Furthermore, $\mathcal{X}_{T}$ is isometrically and holomorphically embedded into $\mathcal{X}$, the rank of $\mathcal{X}_{T}$ equals the rank of $\mathcal{X}$ and as a bounded symmetric domain $\mathcal{X}_{T}$ is realized as

$$
\mathcal{D}_{T}=\mathcal{D} \cap \mathfrak{p}_{T}^{+}
$$

where $\mathfrak{p}_{T}^{+}$are the fixed points of $\operatorname{Ad}(c)^{4}$ in $\mathfrak{p}_{+}$.

Note that the maximal standard polydisk $\mathcal{P}_{0}$ is contained in $\mathcal{D}_{T}$ (see (3.4)), hence also $\sum_{j=1}^{r} E_{\gamma_{j}} \in \check{S}_{\mathcal{D}_{T}} \subset \check{S}_{\mathcal{D}}$. Moreover, for the polynomial $h_{\mathcal{D}}$ which is related to the Bergmann kernel by equation (3.6), we have

$$
h_{\mathcal{D}_{T}}=h_{\left.\mathcal{D}\right|_{\mathfrak{p}_{T}^{+}}} \text {. }
$$

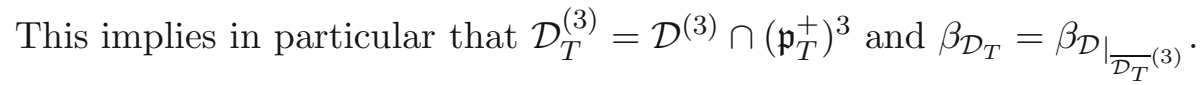

Lemma 5.2. $\mathcal{D}_{T}$ is a maximal (with respect to inclusion) subdomain of tube type in $\mathcal{D}$.

5.1 The Shilov boundary and tube type domains. It is well known that the structure of the Shilov boundary $\breve{S}_{\mathcal{D}}$ detects whether $\mathcal{D}$ is of tube type or not, see for example [KW, Th. 4.9]. Similarly the behavior of the restriction of the Bergmann cocycle to the Shilov boundary detects whether $\mathcal{D}$ is of tube type or not when $\mathcal{D}$ is irreducible. In the general case we have

Proposition 5.3 [BuIoW3, Cor.3.10]. Let

$$
\check{S}^{(3)}:=\left\{\left(z_{1}, z_{2}, z_{3}\right) \in \check{S}^{3}:\left(z_{i}, z_{j}\right) \in \check{S}^{(2)} \text { for all } i \neq j\right\},
$$

the space of triples of pairwise transverse points in $\check{S}$. Then $\check{S}^{(3)} \subset \overline{\mathcal{D}}^{[3]}$ and the Bergmann cocycle $\beta_{\mathcal{D}}$ is well defined and continuous on $\check{S}^{(3)}$. Furthermore,

(1) If $\mathcal{D}$ is of tube type, then

$$
\beta_{\mathcal{D}}\left(\check{S}^{(3)}\right)=\left\{-\frac{\mathrm{r}_{\mathcal{D}}}{2},-\frac{\mathrm{r}_{\mathcal{D}}}{2}+1, \cdots, \frac{\mathrm{r}_{\mathcal{D}}}{2}-1, \frac{\mathrm{r}_{\mathcal{D}}}{2}\right\} .
$$

(2) If $\mathcal{D}$ is irreducible and not of tube type, then

$$
\beta_{\mathcal{D}}\left(\check{S}^{(3)}\right)=\left[-\frac{\mathrm{r}_{\mathcal{D}}}{2}, \frac{\mathrm{r}_{\mathcal{D}}}{2}\right] .
$$


The following relation (see Proposition 5.6) between transverse pairs in the Shilov boundary and maximal subdomains of tube type is important for our later considerations. If $(x, y) \in \check{S}_{\mathcal{D}}^{(2)}$ we define

$$
\left(\check{S}_{\mathcal{D}}\right)_{x, y}:=\left\{z \in \check{S}_{\mathcal{D}}:(z, x) \in \check{S}^{(2)},(z, y) \in \check{S}^{(2)}\right\}
$$

to be the set of points $z \in \check{S}$ which are transverse to $x$ and to $y$. This is an open and dense set in $\check{S}_{\mathcal{D}}$. In the following we shall denote $\sum_{j=1}^{r} E_{\gamma_{j}}$ by $E_{\Lambda}$, where, as in section $3, \Lambda$ refers to the set $\left\{\gamma_{1}, \ldots, \gamma_{r}\right\}$ of strongly orthogonal roots. The following lemma is crucial and follows immediately from the case in which $\mathcal{D}$ is irreducible, which was proven by Clerc and Ørsted as the first step in the proof of Theorem 4.7 in $[\mathrm{C} \varnothing]$.

Lemma $5.4\left[\mathrm{CØ]}\right.$. Let $z \in \check{S}_{\mathcal{D}}$ be transverse to $E_{\Lambda}$ and $-E_{\Lambda}$ with

$$
\left|\beta_{\mathcal{D}}\left(z, E_{\Lambda},-E_{\Lambda}\right)\right|=\frac{\mathrm{r}_{\mathcal{D}}}{2}
$$

Then $z \in \check{S}_{\mathcal{D}_{T}}$.

If $(x, y) \in \check{S}_{\mathcal{D}}^{(2)}$ and $\mathcal{D}^{\prime} \subseteq \mathcal{D}$ is a subdomain of Hermitian type, we define

$$
M_{x, y}\left(\mathcal{D}^{\prime}\right):=\left\{z \in\left(\check{S}_{\mathcal{D}^{\prime}}\right)_{x, y}:\left|\beta_{\mathcal{D}^{\prime}}\left(z, E_{\lambda},-E_{\Lambda}\right)\right|=\frac{\mathrm{r}_{\mathcal{D}}}{2}\right\} .
$$

From the above lemma we now deduce

Proposition 5.5. With the above notation, we have that

$$
M_{E_{\Lambda},-E_{\Lambda}}(\mathcal{D})=M_{E_{\Lambda},-E_{\Lambda}}\left(\mathcal{D}_{T}\right)
$$

and $\check{S}_{\mathcal{D}_{T}}$ is the real Zariski closure in $\check{S}_{\mathcal{D}}$ of $M_{E_{\Lambda},-E_{\Lambda}}(\mathcal{D})$.

Proof. Equation (5.2) follows from Lemma 5.4 and the fact that $\left.\beta_{\mathcal{D}}\right|_{\left(\check{S}_{\mathcal{D}_{T}}\right)}{ }^{(3)}=\beta_{\mathcal{D}_{T}}$.

Since $\mathcal{D}_{T}$ is of tube type, then $M_{E_{\Lambda},-E_{\Lambda}}\left(\mathcal{D}_{T}\right)$ is a nonempty open subset of $\check{S}_{\mathcal{D}_{T}}$, and hence (5.2) implies that the Zariski closure of $M_{E_{\Lambda},-E_{\Lambda}}(\mathcal{D})$ is $\check{S}_{\mathcal{D}_{T}}$

Proposition 5.6. Let $\mathcal{D}$ be a Hermitian symmetric space and $(x, y) \in \check{S}_{\mathcal{D}}^{(2)}$ be a pair of transverse points in its Shilov boundary. Then there exists a unique maximal subdomain $T_{x y} \subset \mathcal{X}$ of tube type with $x, y \in \check{S}_{T_{x y}}$. Moreover, $\check{S}_{T_{x, y}}$ is the real Zariski closure in $\check{S}_{\mathcal{D}}$ of $M_{x, y}(\mathcal{D})$.

Proof. Observe that $\left(E_{\Lambda},-E_{\Lambda}\right)$ are in $\check{S}_{\mathcal{D}_{T}}$ and $\mathcal{D}_{T}$ is a maximal subdomain of tube type. Moreover, since $G_{\mathcal{D}}$ acts transitively on $\check{S}_{\mathcal{D}}^{(2)}$, we obtain the existence statement for every pair $(x, y) \in \check{S}_{\mathcal{D}}^{(2)}$.

Concerning uniqueness, we may assume, again by transitivity of the $G_{\mathcal{D}}$-action on $\check{S}_{\mathcal{D}}^{(2)}$, that $(x, y)=\left(E_{\Lambda},-E_{\Lambda}\right)$. Thus, let $\mathcal{D}^{\prime} \subset \mathcal{D}$ be a maximal subdomain of tube type with $\left(E_{\Lambda},-E_{\Lambda}\right) \in \check{S}_{\mathcal{D}^{\prime}}^{(2)}$. Since $\mathrm{r}_{\mathcal{D}}=\mathrm{r}_{\mathcal{D}^{\prime}}$, we have that $\left.\beta_{\mathcal{D}}\right|_{\left(\check{S}_{\mathcal{D}^{\prime}}\right)^{(3)}}=\beta_{\mathcal{D}^{\prime}}$ and hence

$$
M_{E_{\Lambda},-E_{\Lambda}}\left(\mathcal{D}^{\prime}\right) \subset M_{E_{\Lambda},-E_{\Lambda}}(\mathcal{D})=M_{E_{\Lambda},-E_{\Lambda}}\left(\mathcal{D}_{T}\right)
$$

which implies, upon taking the real Zariski closure and using Proposition 5.5, that $\check{S}_{\mathcal{D}^{\prime}} \subset \check{S}_{\mathcal{D}_{T}}$. On the other hand, $\operatorname{dim} \mathcal{D}^{\prime}=\operatorname{dim} \mathcal{D}_{T}$, which, since $\mathcal{D}^{\prime}$ and $\mathcal{D}_{T}$ are of tube type, implies that $\operatorname{dim} \check{S}_{\mathcal{D}^{\prime}}=\operatorname{dim} \check{S}_{\mathcal{D}_{T}}$ and, together with the previously 
established inclusion, that $\check{S}_{\mathcal{D}^{\prime}}=\check{S}_{\mathcal{D}_{T}}$; this then implies by Proposition 3.11 that $\mathcal{D}^{\prime}=\mathcal{D}_{T}$.

REMARK 5.7. One could prove the uniqueness in Proposition 5.6 also by considering the Lie algebra of the stabilizer of $\left(E_{\Lambda},-E_{\Lambda}\right) \in \check{S}_{\mathcal{D}}^{(2)}$, but for us the characterization of $\check{S}_{T_{x y}}$ obtained as a byproduct of the proof is essential.

Let $\mathcal{T}_{\mathcal{X}}$ be the space of maximal tube type subdomains in $\mathcal{X}$. Then, since all maximal subdomains of tube type are conjugate, $\mathcal{T}_{\mathcal{X}}$ is a homogeneous space under $G_{\mathcal{X}}$. The map

$$
\check{S}_{\mathcal{X}}^{(2)} \rightarrow \mathcal{T}_{\mathcal{X}}
$$

provided by Proposition 5.6 is a $G_{\mathcal{X}}$-equivariant map between $G_{\mathcal{X}}$-homogeneous spaces and hence is real analytic.

\subsection{Structure theorem for tight embeddings, II.}

Theorem 5.8. Let H, $G$ be Lie groups of Hermitian type with associated symmetric spaces $\mathcal{Y}$ and $\mathcal{X}$. Let $\rho: H \rightarrow G$ be a continuous tight homomorphism and $f: \mathcal{Y} \rightarrow \mathcal{X}$ the induced $\rho$-equivariant tight map. Then,

(1) If $\mathcal{Y}$ is of tube type, then there exists a unique maximal tube type subdomain $T \subset \mathcal{X}$ such that $f(\mathcal{Y}) \subset T$ and $\rho(H)$ preserves $T$.

(2) If $\rho$ has finite kernel and $\mathcal{X}$ is of tube type, then $\mathcal{Y}$ is of tube type.

We shall need the following.

LEMmA 5.9. Under the assumption of Theorem 5.8, let $\check{f}: \check{S}_{\mathcal{Y}} \rightarrow \check{S}_{\mathcal{X}}$ be the (continuous) equivariant map given by Theorem 4.1, and let

$$
f^{*}\left(\omega_{\mathcal{X}}\right)=\sum_{i=1}^{n} \lambda_{i} \omega_{\mathcal{Y}, i}
$$

Then for all $(x, y, z) \in \check{S}^{(3)}$, we have

$$
\beta_{\mathcal{X}}(\check{f}(x), \check{f}(y), \check{f}(z))=\sum_{i=1}^{n} \lambda_{i} \beta_{\mathcal{Y}_{i}}\left(x_{i}, y_{i}, z_{i}\right) .
$$

In particular, if $f$ is moreover positive and $\beta \mathcal{Y}(x, y, z)=\mathrm{r}_{\mathcal{Y}} / 2$, then

$$
\beta_{\mathcal{X}}(f(x), f(y), f(z))=\frac{\mathrm{r} \mathcal{X}}{2} .
$$

Proof. Let $0 \in \mathcal{D}_{\mathcal{Y}}$ and $r_{0, x}, r_{0, y}$ and $r_{0, z}$ be the geodesic rays given by Proposition 3.4 ; then we know that

$$
\check{f}(x)=\lim _{t \rightarrow \infty} f\left(r_{0, x}(t)\right),
$$

and analogously for $y$ and $z$. Writing $x=\left(x_{1}, \ldots, x_{n}\right)$ and $y$ and $z$ in coordinates, we have

$$
\begin{aligned}
\beta_{\mathcal{X}}\left(f\left(r_{0, x}(t)\right), f\left(r_{0, y}(t)\right), f\left(r_{0, z}(t)\right)\right) & =\int_{\Delta\left(r_{0, x}(t), r_{0, y}(t), r_{0, z}(t)\right)} f^{*}\left(\omega_{\mathcal{X}}\right) \\
& =\sum_{i=1}^{n} \lambda_{i} \beta_{\mathcal{Y}_{i}}\left(r_{0, x_{i}}(t), r_{0, y_{i}}(t), r_{0, z_{i}}(t)\right)
\end{aligned}
$$


and by using (5.3) and the fact that the normalized Bergmann cocycles extend continuously to $\check{S}_{\mathcal{Y}_{i}}^{(3)}$ and $\check{S}_{\mathcal{X}}^{(3)}$, we conclude the first claim.

Now assume that $f$ is positive; then if $\beta_{\mathcal{Y}}(x, y, z)=\mathrm{r}_{\mathcal{Y}} / 2$, then $\beta_{\mathcal{Y}_{i}}\left(x_{i}, y_{i}, z_{i}\right)=$ $\mathrm{r}_{\mathcal{Y}_{i}} / 2$ and hence

$$
\beta_{\mathcal{X}}(\check{f}(x), \check{f}(y), \check{f}(z))=\sum_{i=1}^{n} \lambda_{i} \frac{\mathrm{r}_{\mathcal{Y}_{i}}}{2}=\frac{\mathrm{r}_{\mathcal{X}}}{2},
$$

where the last equality follows from Corollary 2.17 and the fact that $f$ is positive, that is $\lambda_{i} \geq 0$.

Proof. By changing complex structure on $\mathcal{Y}$ we may assume that $f$ is positive (Lemma 4.10). Let $\left(\check{S}_{\mathcal{Y}}\right)_{x}$ be the set of points in $\mathcal{Y}$ transverse to $x$ so that $\left(\check{S}_{\mathcal{Y}}\right)_{x, y}=$ $\left(\check{S}_{\mathcal{Y}}\right)_{x} \cap\left(\check{S}_{\mathcal{Y}}\right)_{y}$, and let us consider the set $M_{x, y}(\mathcal{Y})$ defined in (5.1). Let $\check{f}: \check{S}_{\mathcal{Y}} \rightarrow \check{S}_{\mathcal{X}}$ be the equivariant extension of $f$ given by Theorem 4.1. Since $f$ is tight and positive, we have that for every $z \in M_{x, y}(\mathcal{Y})$

$$
\left|\beta_{\mathcal{X}}(\check{f}(x), \check{f}(y), \check{f}(z))\right|=\frac{\mathrm{r}_{\mathcal{Y}}}{2}
$$

(see Lemma 5.9), and hence

by Proposition 5.5, and thus,

$$
\check{f}(z) \in \check{S}_{T_{\check{f}(x), \check{f}(y)}}
$$

$$
T_{\check{f}(x), \check{f}(y)}=T_{\check{f}(x), \check{f}(z)}
$$

by the uniqueness statement in Proposition 5.6.

Let $\mathcal{T}_{\mathcal{X}}$ be the conjugacy class of maximal tube type domains seen as a Ghomogeneous space and hence as a real analytic variety. The map

$$
\begin{aligned}
(\check{S})_{x} & \longrightarrow \mathcal{T}_{\mathcal{X}} \\
z & \longmapsto T_{\check{f}(x), \check{f}(z)}
\end{aligned}
$$

is real analytic and constant on the subset $M_{x, y}(\mathcal{Y}) \subset(\check{S} \mathcal{Y})_{x}$; but since $\mathcal{Y}$ is of tube type, $M_{x, y}(\mathcal{Y})$ is open, $\left(\check{S}_{\mathcal{Y}}\right)_{x}$ is connected and hence the map (5.4) is constant on $\left(\check{S}_{\mathcal{Y}}\right)_{x}$

Let now $\left(x_{1}, y_{1}\right)$ and $\left(x_{2}, y_{2}\right)$ be arbitrary elements in $\left(\check{S}_{\mathcal{Y}}\right)^{(2)}$ and choose $z \in\left(\check{S}_{\mathcal{Y}}\right)_{x_{1}, x_{2}}$. Then we have

$$
T_{\check{f}\left(x_{1}\right), \check{f}\left(y_{1}\right)}=T_{\check{f}\left(x_{1}\right), \check{f}(z)}=T_{\check{f}\left(x_{2}\right), \check{f}(z)}=T_{\check{f}\left(x_{2}\right), \check{f}\left(y_{2}\right)}
$$

which shows that the map

$$
\begin{aligned}
& \left(\check{S}_{\mathcal{Y}}\right)^{(2)} \longrightarrow \mathcal{T}_{\mathcal{X}} \\
& (x, y) \longmapsto T_{\check{f}(x), \check{f}(y)}
\end{aligned}
$$

is constant and hence its constant value $T \subset \mathcal{X}$ is $\rho(H)$-invariant. Since $\check{f}(x) \in \check{S}_{T_{\check{f}(x), \check{f}(y)}}$, we also deduce that $\check{f}\left(\check{S}_{\mathcal{Y}}\right) \subset \check{S}_{T}$.

Now, by Theorem 4.1 we know that the centralizer of $\rho(H)<G$ in $G$ is compact; this implies that, given any maximal compact subgroup $K<H$, there is a unique point $x_{K} \in \mathcal{X}$ which is $\rho(K)$-fixed. Since $\rho(H)$ leaves $T$ invariant, this implies that $x_{K} \in T$ and hence that $f(\mathcal{Y}) \subset T$. 
For the second statement, observe that if $\mathcal{X}$ is of tube type, then $\beta_{\mathcal{X}}$ takes on finitely many values; since $\rho$ has finite kernel, this implies that $\lambda_{i} \neq 0$ for all $1 \leq i \leq n$ and hence each $\beta_{\mathcal{Y}_{i}}$ takes on finitely many values on $\check{S}_{\mathcal{Y}_{i}}^{(3)}$, and this, together with Proposition 5.3, implies that $\mathcal{Y}_{i}$ is of tube type.

\section{Extensions to Groups of Type (RH)}

Here we indicate the argument extending Theorems 4.1 and 5.8 to Lie groups of type $(\mathrm{RH})$. The study of tight homomorphisms of groups of type $(\mathrm{RH})$ can be reduced to the study of homomorphisms of groups of Hermitian type. In fact, let $G_{1}, G_{2}$ be groups of type $(\mathrm{RH})$ and let $\rho: G_{1} \rightarrow G_{2}$ be a continuous homomorphism. We have the inclusion $\rho\left(G_{1, n c}\right) \subset G_{2, n c}$ and hence the commutative diagram

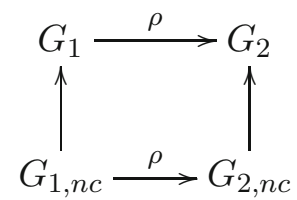

hence $\rho_{\mathrm{b}}^{*}\left(\kappa_{G_{2}}^{\mathrm{b}}\right)_{\left.\right|_{G_{1, n c}}}=(\rho)_{\mathrm{b}}^{*}\left(\kappa_{\left.G_{2}\right|_{G_{2}, n c}}\right)$. From this and Corollary 2.3 we deduce the equalities

$$
\left\|\rho_{\mathrm{b}}^{*}\left(\kappa_{G_{2}}^{\mathrm{b}}\right)\right\|=\left\|\rho_{\mathrm{b}}^{*}\left(\kappa_{\left.G_{2}\right|_{G_{2, n c}}}^{\mathrm{b}}\right)\right\| \text { and }\left\|\kappa_{G_{2}}^{\mathrm{b}}\right\|=\left\|\kappa_{\left.G_{2}\right|_{G_{2, n c}}}\right\|,
$$

from which it follows that $\rho$ is tight if and only of $\rho_{\left.\right|_{G_{1, n c}}}$ is tight.

From this and Theorems 4.1 and 5.8 we readily deduce the following:

Theorem 6.1. Let $H, G$ be Lie groups of type $(R H), \rho: H \rightarrow G$ a continuous tight homomorphism and $f: \mathcal{D}^{\prime} \rightarrow \mathcal{D}$ the corresponding $\rho$-equivariant tight totally geodesic map. Then $f$ extends continuously to a $\rho$-equivariant map

$$
\check{f}: \check{S}_{\mathcal{D}^{\prime}} \rightarrow \check{S}_{\mathcal{D}} \text {. }
$$

Moreover the centralizer $\mathcal{Z}_{G}(\rho(H))$ is compact.

Theorem 6.2. Let $H, G$ be Lie groups of type $(R H)$ with associated symmetric spaces $\mathcal{Y}$ and $\mathcal{X}$. Let $\rho: H \rightarrow G$ be a continuous tight homomorphism and $f: \mathcal{Y} \rightarrow \mathcal{X}$ the induced $\rho$-equivariant tight map. Then,

(1) If $\mathcal{Y}$ is of tube type, then there exists a unique maximal tube type subdomain $T \subset \mathcal{X}$ such that $f(\mathcal{Y}) \subset T$ and $\rho(H)$ preserves $T$.

(2) If $\rho$ has compact kernel and $\mathcal{X}$ is of tube type, then $\mathcal{Y}$ is of tube type.

\section{Structure Theorem for Tight Homomorphisms}

In this section we prove the main structure theorem for tight homomorphisms.

Theorem 7.1. Let $L$ be a locally compact group, $\mathbf{G}$ a connected algebraic group defined over $\mathbb{R}$ such that $G=\mathbf{G}(\mathbb{R})^{\circ}$ is of type $(R H)$, and $\rho: L \rightarrow G$ a continuous tight homomorphism. Then:

(1) The Zariski closure $\mathbf{H}:=\overline{\rho(L)}^{Z}$ is reductive. 
(2) The centralizer $\mathcal{Z}_{G}(H)$ of $H:=\overline{\rho(L)}^{Z}(\mathbb{R})^{\circ}$ is compact.

(3) The group $H$ is of type $(R H)$ and the symmetric space $\mathcal{Y}$ corresponding to $H$ is Hermitian.

(4) There is a unique complex structure on $\mathcal{Y}$ such that the embedding $H \rightarrow G$ is tight and positive.

Proof. Set $H:=\overline{\rho(L)}^{Z}(\mathbb{R})^{\circ}$. Then the inclusion $H \rightarrow G$ is tight. Let

$$
H=H^{s s} \cdot R
$$

be the decomposition of $H$, where $R$ is the amenable radical and $H^{s s}$ is a semisimple connected Lie group with finite center and no compact factors. Then it follows from Corollary 2.3 that the inclusion $H^{s s} \rightarrow G$ is tight. Let

$$
H^{s s}=H_{1} \cdots H_{n}
$$

be the decomposition of $H^{s s}$ into almost simple factors, and let $H_{1}, \ldots, H_{l}, l \leq n$, be the almost simple factors of $H^{s s}$ for which the restriction $\left.\kappa_{G}^{\mathrm{b}}\right|_{H_{i}} \in \mathrm{H}_{\mathrm{cb}}^{2}\left(H_{i}, \mathbb{R}\right)$ is nonzero. Then

$$
\left\|\kappa_{G}^{\mathrm{b}}\right\|=\left\|\left.\kappa_{G}^{\mathrm{b}}\right|_{H^{s s}}\right\|=\sum_{i=1}^{l}\left\|\left.\kappa_{G}^{\mathrm{b}}\right|_{H_{i}}\right\|
$$

and the inclusion

$$
H_{1} \ldots H_{l} \rightarrow G_{n c}
$$

is tight. Let $\mathcal{X}$ be the symmetric space associated to $G_{n c}, \mathcal{Y}_{i}$ the symmetric space associated to $H_{i}, 1 \leq i \leq l$, and $\mathcal{Y}_{1} \times \cdots \times \mathcal{Y}_{l} \rightarrow \mathcal{X}$ the corresponding tight embedding.

Then, by Theorem 4.1 , the centralizer $\mathcal{Z}_{G_{n c}}\left(H_{1} \ldots H_{l}\right)$ is compact, which implies first that $\ell=n$, that is $\mathcal{Z}_{G_{n c}}\left(H^{s s}\right)$ is compact and hence that $\mathcal{Z}_{G}(H)$ is compact. Now, if $H$ were not reductive, it would be contained in a proper parabolic subgroup of $G$ and hence $\mathcal{Z}_{G}\left(H^{s s}\right)$ would be noncompact. Hence $H$ is reductive and, since $\mathcal{Z}_{G}\left(H^{s s}\right)$ is compact and $\mathcal{Y}_{1} \times \ldots \mathcal{Y}_{n}$ is Hermitian symmetric, the group $H$ is of type $(\mathrm{RH})$.

Finally, (4) follows from Lemma 4.10.

From Theorem 7.1 we can now deduce the following

Theorem 7.2. Let $\Gamma$ be a countable discrete group with probability measure $\theta$ and let $\mathbf{G}$ be a semisimple real algebraic group such that $G:=\mathbf{G}(\mathbb{R})^{\circ}$ is of type $(R H)$. If $(B, \nu)$ is a Poisson boundary for $(\Gamma, \theta)$ and $\rho: \Gamma \rightarrow G$ is a tight homomorphism, then there exists a $\rho$-equivariant measurable map

$$
\varphi: B \rightarrow \check{S}_{\mathcal{X}}
$$

Proof. Let $\mathbf{H}$ be the Zariski closure of $\rho(\Gamma)$. By Theorem 7.1 the symmetric space $\mathcal{Y}$ associated to $H:=\mathbf{H}(\mathbb{R})^{\circ}$ is Hermitian symmetric and we fix a complex structure such that the embedding $\mathcal{Y} \rightarrow \mathcal{X}$ is tight and positive. Theorem 4.1 gives the existence of a $\rho$-equivariant map $\check{f}$ between the corresponding Shilov boundaries

$$
\check{f}: \check{S}_{\mathcal{Y}} \rightarrow \check{S}_{\mathcal{X}} \text {. }
$$

Let $\mathbf{Q}_{H}<\mathbf{H}$ be a maximal parabolic subgroup defined over $\mathbb{R}$ such that $\check{S}_{\mathcal{Y}} \cong$ $\mathbf{H}(\mathbb{R}) / \mathbf{Q}_{H}(\mathbb{R})$, and let $\mathbf{P}_{H}<\mathbf{Q}_{H}$ be a minimal parabolic subgroup defined over $\mathbb{R}$ 
contained in $\mathbf{Q}_{H}$, so that we have an equivariant map

$$
\mathbf{H}(\mathbb{R}) / \mathbf{P}_{H}(\mathbb{R}) \rightarrow \mathbf{H}(\mathbb{R}) / \mathbf{Q}_{H}(\mathbb{R}) \cong \check{S}_{\mathcal{Y}} .
$$

Since $\rho: \Gamma \rightarrow H$ has Zariski dense image [BuIoW3, Th.4.7] implies the existence of a $\rho$-equivariant measurable boundary map

$$
\varphi_{0}: B \rightarrow \mathbf{H}(\mathbb{R}) / \mathbf{P}_{H}(\mathbb{R})
$$

which composed with the maps in (7.1) and (7.2) provides the $\rho$-equivariant map $\varphi: B \rightarrow \check{S}$.

\section{A Criterion for Tightness}

To get a simple criterion when a totally geodesic embedding is tight let us recall the relation between totally geodesic embeddings and Lie algebra homomorphisms.

Let $G_{1}, G_{2}$ be connected semisimple Lie groups with finite center and no compact factors and $\mathcal{X}_{1}, \mathcal{X}_{2}$ be the corresponding symmetric spaces. Fix two base points $x_{j} \in \mathcal{X}_{j}, j=1,2$, and let $\mathfrak{g}_{j}=\mathfrak{k}_{j} \oplus \mathfrak{p}_{j}$ be the corresponding Cartan decompositions of $\mathfrak{g}_{j}=\operatorname{Lie}\left(G_{j}\right)$. Then every totally geodesic embedding $f: \mathcal{X}_{1} \rightarrow \mathcal{X}_{2}$ with $f\left(x_{1}\right)=x_{2}$ induces a Lie algebra homomorphism $\rho: \mathfrak{g}_{1} \rightarrow \mathfrak{g}_{2}$ which respects the Cartan decompositions. Conversely, any Lie algebra homomorphism $\rho: \mathfrak{g}_{1} \rightarrow \mathfrak{g}_{2}$ respecting the Cartan decompositions gives rise to a totally geodesic embedding $f: \mathcal{X}_{1} \rightarrow \mathcal{X}_{2}$ with $f\left(x_{1}\right)=x_{2}$.

Let $\mathcal{X}$ be a Hermitian symmetric space with a fixed complex structure $\mathcal{J}_{\mathcal{X}}$ and let $Z_{\mathcal{J} \mathcal{X}} \in \mathcal{Z}(\mathfrak{k})$ be the element in the center of $\mathfrak{k}$ such that $\operatorname{ad}\left(Z_{\mathcal{J}_{\mathcal{X}}}\right)_{\left.\right|_{\mathfrak{p}}}$ induces the complex structure $\mathcal{J}_{\mathcal{X}}$ on $T_{x_{0}} \mathcal{X} \cong \mathfrak{p}$. The restriction of the Killing form $\mathfrak{B}$ on $\mathfrak{g}$ to $\mathfrak{k}$ is a negative definite symmetric bilinear form. Let

$$
\mathfrak{k}=\mathbb{R} Z_{\mathcal{J}_{\mathcal{X}}} \oplus \mathbb{R} Z_{\mathcal{J}_{\mathcal{X}}}^{\perp}
$$

be the orthogonal decomposition of $\mathfrak{k}$ with respect to $\mathfrak{B}_{\mid \mathfrak{k}}$. We identify $\mathbb{R} Z_{\mathcal{J}_{\mathcal{X}}}$ with $\mathbb{R}$ by sending $Z_{\mathcal{J}_{\mathcal{X}}}$ to $i$. Then the orthogonal projection onto $\mathbb{R} Z_{\mathcal{J}_{\mathcal{X}}}$ defines a homomorphism $\lambda_{Z_{\mathcal{J}}} \in \operatorname{Hom}(\mathfrak{k}, i \mathbb{R})$.

To relate tightness of a totally geodesic embedding with properties of the corresponding Lie algebra homomorphism we make use of the isomorphism

$$
\operatorname{Hom}(\mathfrak{k}, i \mathbb{R}) \rightarrow \Omega^{2}(\mathcal{X})^{G_{\mathcal{X}}} \rightarrow \mathrm{H}_{\mathrm{cb}}^{2}\left(G_{\mathcal{X}}, \mathbb{R}\right),
$$

where the first map associates to a homomorphism $\lambda \in \operatorname{Hom}(\mathfrak{k}, i \mathbb{R})$ the unique $G_{\mathcal{X}^{-}}$ invariant differential form on $\mathcal{X}$ whose value at $x_{0}$ is

for $X, Y \in \mathfrak{p} \cong T_{x_{0}} \mathcal{X}$.

$$
\left(\omega_{\lambda}\right)_{x_{0}}(X, Y):=\frac{1}{4 \pi i} \lambda([X, Y])
$$

Let $\mathcal{X}_{1}, \mathcal{X}_{2}$ be two Hermitian symmetric spaces with complex structures $\mathcal{J}_{\mathcal{X}_{j}}$ given by $Z_{j}=Z_{\mathcal{J}_{j}} \in \mathcal{Z}\left(\mathfrak{k}_{j}\right), j=1,2$. Let $f: \mathcal{X}_{1} \rightarrow \mathcal{X}_{2}$ be a totally geodesic embedding, $f\left(x_{1}\right)=x_{2}$, and $\rho: \mathfrak{g}_{1} \rightarrow \mathfrak{g}_{2}$ the corresponding Lie algebra homomorphism. Let $\mathbb{D}$ be the Poincaré disk and $Z_{\mathbb{D}} \in \mathfrak{s o}(2) \subset \mathfrak{s l}(2, \mathbb{R})$ the element which induces the standard complex structure on $\mathbb{D}$. Let $d_{j}: \mathbb{D} \rightarrow \mathcal{X}_{j}, j=1,2$ be diagonal disks with $d_{j}(0)=x_{j}$ and $\rho_{j}: \mathfrak{s l}(2, \mathbb{R}) \rightarrow \mathfrak{g}_{j}$ the corresponding Lie algebra homomorphisms. 
Lemma 8.1. The embedding $f: \mathcal{X}_{1} \rightarrow \mathcal{X}_{2}$ is tight and positive if and only if

$$
\lambda_{Z_{2}}\left(\rho\left(\rho_{1}\left(Z_{\mathbb{D}}\right)\right)\right)=\lambda_{Z_{2}}\left(\rho_{2}\left(Z_{\mathbb{D}}\right)\right) .
$$

Proof. We start by showing that since the embedding $d_{1}: \mathbb{D} \rightarrow \mathcal{X}_{1}$ is tight and positive, then $f: \mathcal{X}_{1} \rightarrow \mathcal{X}_{2}$ is tight and positive if and only if $h:=f \circ d_{1}: \mathbb{D} \rightarrow \mathcal{X}_{2}$ is tight and positive. In fact, let $\mathcal{X}_{1}=\mathcal{X}_{1,1} \times \cdots \times \mathcal{X}_{1, n}$ be the decomposition of $\mathcal{X}$ into irreducible subspaces and let

$$
f^{*}\left(\omega_{\mathcal{X}_{2}}\right)=\sum_{i=1}^{n} \lambda_{i} \omega_{\mathcal{X}_{1}, i}
$$

Then

$$
h^{*}\left(\omega_{\mathcal{X}_{2}}\right)=\sum_{i=1}^{n} \lambda_{i} d_{1}^{*}\left(\omega_{\mathcal{X}_{1}, i}\right)=\sum_{i=1}^{n} \lambda_{i} \mathrm{r}_{\mathcal{X}_{1, i}} \omega_{\mathbb{D}},
$$

where the last inequality follows from the fact that $d_{1}$ is tight and positive. If $h$ is tight and positive, then

$$
h^{*}\left(\omega_{\mathcal{X}_{2}}\right)=\mathrm{r}_{\mathcal{X}_{2}} \omega_{\mathbb{D}}
$$

so that

$$
\sum_{i=1}^{n} \lambda_{i} \mathrm{r}_{\mathcal{X}_{1, i}}=\mathrm{r}_{\mathcal{X}_{2}}
$$

Since $f$ is norm decreasing then

$$
\sum_{i=1}^{n}\left|\lambda_{i}\right| \mathrm{r}_{\mathcal{X}_{1, i}} \leq \mathrm{r}_{\mathcal{X}_{2}}
$$

which together with (8.2) implies that $f$ is positive and, by Corollary 2.17, tight.

Let $\omega_{\lambda_{Z_{2}}} \in \Omega^{2}\left(\mathcal{X}_{2}\right)^{G_{2}}$ be the differential form corresponding to $\lambda_{Z_{2}}$. Then, since $\Omega^{2}(\mathbb{D})^{\mathrm{PSL}(2, \mathbb{R})}=\mathbb{R} \omega_{\mathbb{D}}$ is one dimensional

$$
h^{*} \omega_{\lambda_{Z_{2}}}=\frac{\lambda_{Z_{2}}\left(\rho\left(\rho_{1}\left(Z_{\mathbb{D}}\right)\right)\right)}{\lambda_{Z_{2}}\left(\rho_{2}\left(Z_{\mathbb{D}}\right)\right)} d_{2}^{*} \omega_{\lambda_{Z_{2}}} .
$$

But since $d_{2}$ is tight and positive, $h$ is tight and positive if and only if the proportionality constant is equal to 1 .

Lemma 8.1 gives a criterion for tightness which takes on a particular nice form when $\mathcal{X}_{2}$ is of tube type. Recall from [KW, Prop. 3.12.] that a Hermitian symmetric space $\mathcal{X}_{2}$ is of tube type if and only if there exists a diagonal disk $d_{2}: \mathbb{D} \rightarrow \mathcal{X}_{2}$ such that the corresponding Lie algebra homomorphism satisfies $\rho_{2}\left(Z_{\mathbb{D}}\right)=Z_{2}$.

Corollary 8.2. Let $\mathcal{X}_{1}, \mathcal{X}_{2}$ be Hermitian symmetric spaces of tube type. A totally geodesic embedding $f: \mathcal{X}_{1} \rightarrow \mathcal{X}_{2}$ is tight and positive if and only if the corresponding Lie algebra homomorphism $\rho: \mathfrak{g}_{1} \rightarrow \mathfrak{g}_{2}$ satisfies

$$
\lambda_{Z_{2}}\left(\rho\left(Z_{1}\right)\right)=1
$$

Proof. Since $\mathcal{X}_{1}, \mathcal{X}_{2}$ are of tube type, we can choose the tight holomorphic disks $\mathrm{d}_{j}: \mathbb{D} \rightarrow \mathcal{X}_{j}, j=1,2$, such that the corresponding Lie algebra homomorphisms $\rho_{j}: \mathfrak{s l}(2, \mathbb{R}) \rightarrow \mathfrak{g}_{j}$ satisfy $\rho_{j}\left(Z_{\mathbb{D}}\right)=Z_{j}$. Then $\lambda_{Z_{2}}\left(\rho_{2}\left(Z_{\mathbb{D}}\right)\right)=\lambda_{Z_{2}}\left(Z_{2}\right)=1$ and Lemma 8.1 implies the claim. 
Let us compare this criterion for tightness with the characterization of Lie algebra homomorphisms corresponding to holomorphic totally geodesic embeddings.

Definition 8.3 [S2]. A homomorphism $\rho: \mathfrak{g}_{1} \rightarrow \mathfrak{g}_{2}$ is said to be of type

(H1) if $\operatorname{ad}\left(\rho\left(Z_{1}\right)\right)=\operatorname{ad}\left(Z_{2}\right)$.

(H2) if $\rho\left(Z_{1}\right)=Z_{2}$.

$\left(\mathrm{H} 2^{\prime}\right)$ if $\rho$ is (H1) and the induced holomorphic totally geodesic map $\mathcal{D}_{1} \rightarrow \mathcal{D}_{2}$ maps the Shilov boundary of $\mathcal{D}_{1}$ into the Shilov boundary of $\mathcal{D}_{2}$.

Lie algebra homomorphisms of type (H1) are in one-to-one correspondence with holomorphic totally geodesic embeddings $\mathcal{X}_{1} \rightarrow \mathcal{X}_{2}$.

With these definitions, Theorem 4.1 implies

Corollary 8.4. Assume that $f: \mathcal{X}_{1} \rightarrow \mathcal{X}_{2}$ is a holomorphic tight embedding. Then the corresponding Lie algebra homomorphism $\rho: \mathfrak{g}_{1} \rightarrow \mathfrak{g}_{2}$ is an (H2') homomorphism.

Corollary 8.2 together with [S2, Prop. 10.12] implies the following.

Corollary 8.5. Suppose $\mathcal{X}_{1}, \mathcal{X}_{2}$ are Hermitian symmetric spaces of tube type. Then $f: \mathcal{X}_{1} \rightarrow \mathcal{X}_{2}$ is a tight and holomorphic embedding if and only if the corresponding homomorphism of Lie algebras $\rho: \mathfrak{g}_{1} \rightarrow \mathfrak{g}_{2}$ is an (H2)-homomorphism.

REMARK 8.6. When $\mathfrak{g}_{1}, \mathfrak{g}_{2}$ are not of tube type, the property of being an (H2)Lie algebra homomorphism does not imply tightness: for $\mathfrak{s u}(1, n)=\operatorname{Aut}(V, h)$ the representations of $\mathfrak{s u}(1, n)$ on $\Lambda^{k}(V)$ preserving the Hermitian form induced by $h$ are always (H2) [S2, p. 188], but we will see below that they are tight only for $k=1$.

\subsection{Examples.}

EXAMPlE 8.7. By Corollary 8.5 (H2) Lie algebra homomorphisms give examples of diagonal embeddings if the Hermitian symmetric spaces are of tube type. All (H2) Lie-algebra homomorphisms were classified by Satake [S1] and Ihara [I1,2].

If we are actually interested in the existence of tight homomorphism of Lie groups of Hermitian type the problem becomes more complicated since the Lie algebra homomorphism might only lift to a Lie group homomorphism of a finite cover of the adjoint Lie group. Satake showed in [S1] (see also [S2, Ch. IV]) that some of the those (H2) Lie algebra homomorphism lift to Lie group homomorphisms, for example,

$$
\begin{array}{ll}
\tau: & \operatorname{SU}(n, n) \rightarrow \operatorname{Sp}(4 n, \mathbb{R}), \\
\tau: & \operatorname{SO}^{*}(4 n) \rightarrow \operatorname{Sp}(8 n, \mathbb{R}), \\
\tau: & \operatorname{Spin}(2, n) \rightarrow \operatorname{Sp}(2 m, \mathbb{R}), \text { where } m \text { depends on } n \bmod 8,
\end{array}
$$

are tight homomorphisms.

EXAMPLE 8.8. An important and interesting tight embedding that is not holomorphic is the embedding of the Poincaré disk into the Siegel upper half space obtained from the $2 n$-dimensional irreducible representation $\mathfrak{s l}(2, \mathbb{R}) \rightarrow \mathfrak{s p}(2 n, \mathbb{R})$.

Proposition 8.9. The homomorphism $\rho: \mathfrak{s l}(2, \mathbb{R}) \rightarrow \mathfrak{s p}(2 n, \mathbb{R})$ given by the $2 n$-dimensional irreducible representation of $\mathfrak{s l}(2, \mathbb{R})$ is tight. 
Proof. Let $Z_{2}$ and $Z_{2 n}$ be generators of the center of the maximal compact Lie subalgebras in $\mathfrak{s l}(2, \mathbb{R})$ and $\mathfrak{s p}(2 n, \mathbb{R})$ respectively. Let $\lambda$ be the homomorphism $\operatorname{Hom}\left(\mathfrak{k}_{2 n}, i \mathbb{R}\right)$ given by the orthogonal projection onto $\mathbb{R} \cdot Z_{2 n}$. Then we have to determine $\lambda\left(\rho\left(Z_{2}\right)\right)$.

Let $V=\mathbb{R}_{2 n-1}[x, y]$ be the vector space of homogeneous polynomials of degree $2 n-1$ in two variables $x, y$, with a basis is given by $\left(P_{0}, \ldots P_{m}\right), m=2 n-1$, where $P_{k}(x, y)=x^{m-k} y^{k}$. The $2 n$-dimensional irreducible representation of $\mathfrak{s l}(2, \mathbb{R})$ is given by the following action: Let $X=\left(\begin{array}{cc}a & b \\ c & -a\end{array}\right) \in \mathfrak{s l}(2, \mathbb{R})$, then

$$
\rho(X) P_{k}(x, y)=a(m-2 k) P_{k}+b(m-k) P_{k+1}+c k P_{k-1} .
$$

This action preserves the skew symmetric bilinear form $\langle.,$.$\rangle on V$, defined by $\left\langle P_{k}, P_{l}\right\rangle=(-1)^{k}\left(\begin{array}{c}k+l \\ k\end{array}\right)^{-1} \delta_{m-k, l}$ and gives rise to the irreducible representation

$$
\rho: \mathfrak{s l}(2, \mathbb{R}) \rightarrow \mathfrak{s p}(2 n, \mathbb{R}),
$$

into the Lie algebra of the symplectic group $\operatorname{Sp}(V,\langle.,\rangle$.$) . The map J$ defined by $J P_{k}=(-1)^{k} P_{m-k}$ gives a complex structure on $V$ and the element in the center of $\mathfrak{k}_{2 n} \subset \mathfrak{s p}(2 n, \mathbb{R})$ which induces the complex structure on $\mathfrak{p}_{2 n} \subset \mathfrak{s p}(2 n, \mathbb{R})$ via the adjoint action is $Z_{2 n}=\frac{1}{2} \mathrm{~J}$.

The image of the element

$$
Z_{2}=\frac{1}{2}\left(\begin{array}{cc}
0 & -1 \\
1 & 0
\end{array}\right)
$$

is given by $\rho\left(Z_{2}\right) P_{k}=\frac{1}{2}\left((k-m) P_{k+1}+k P_{k-1}\right)$. Decomposing $\rho\left(Z_{2}\right)=\lambda\left(\rho\left(Z_{2}\right)\right) Z_{2 n}$ $\bmod Z_{2 n}^{\perp}$, we get

$$
Z_{2 n} \rho\left(Z_{2}\right)=-\frac{\lambda}{4} \operatorname{Id}_{V} \bmod Z_{2 n}^{\perp}
$$

Since $\operatorname{tr}\left(Z_{2 n} Z_{2 n}^{\perp}\right)=0$, we have that $\operatorname{tr}\left(Z_{2 n} \rho\left(Z_{2}\right)\right)=-\frac{\lambda}{4} \operatorname{dim}(V)$. Now $Z_{2 n} \rho\left(Z_{2}\right) P_{k}=$ $\frac{1}{4}(-1)^{k+1}\left[(m-k) P_{m-k-1}-k P_{m-k+1}\right]$. Thus the diagonal terms are

$$
\frac{1}{4}(-1)^{n}(2 n-1-n+1) \quad \text { for } k=n-1 \quad \text { and } \quad \frac{1}{4}(-1)^{n} n \quad \text { for } k=n .
$$

Hence

$$
\left|\operatorname{tr}\left(Z_{2 n} \rho\left(Z_{2}\right)\right)\right|=\frac{n}{2}=\frac{1}{4} \operatorname{dim}(V)=\left|\operatorname{tr}\left(Z_{2 n} Z_{2 n}\right)\right|,
$$

and $|\lambda|=1$.

Proposition 8.10. The irreducible representation $\rho: \mathfrak{s u}(1,2) \rightarrow \mathfrak{s u}(2,4)$ is not tight.

Proof. Let $V$ be a 3-dimensional complex vector space with Hermitian form of signature $(1,2)$. The irreducible representation $\rho: \mathfrak{s u}(1,2) \rightarrow \mathfrak{s u}(2,4)$ is the representation given by the action of $\mathfrak{s u}(1,2)$ on $\operatorname{Sym}^{2}(V)$ with the induced Hermitian form. Let $Z_{\mathfrak{s u}(2,4)}$ be the generator of the center of the maximal compact subalgebra on $\mathfrak{s u}(2,4)$ and $Z_{\mathfrak{s u}(2,2)}$ the generator of the center of the maximal compact subalgebra of the tightly embedded subalgebra $\mathfrak{s u}(2,2) \subset \mathfrak{s u}(2,4)$. Then $\operatorname{tr}\left(Z_{\mathfrak{s u}(2,4)} Z_{\mathfrak{s u}(2,2)}\right)=-1$, so the representation $\rho: \mathfrak{s u}(1,2) \rightarrow \mathfrak{s u}(2,4)$ is tight if and only if $\left|\operatorname{tr}\left(Z_{\mathfrak{s u}(2,4)} \rho\left(Z_{\mathfrak{s u}(1,1)}\right)\right)\right|=1$, where $Z_{\mathfrak{s u}(1,1)}$ is a generator of the center of the maximal compact subalgebra of $\mathfrak{s u}(1,1) \subset \mathfrak{s u}(1,2)$. A direct computation shows that $\left|\operatorname{tr}\left(Z_{\mathfrak{s u}(2,4)} \rho\left(Z_{\mathfrak{s u}(1,1)}\right)\right)\right|=1 / 6$, thus $\rho$ is not tight. 
EXAMPlE 8.11. Considering a complex vector space $V_{\mathbb{C}}$ of dimension $(1+n)$ with a Hermitian form of signature $(1, n)$ as real vector space $V_{\mathbb{R}}$ of dimension $(2+n)$ with a quadratic form of signature $(2, n)$ provides a natural embedding $\mathrm{SU}(1, n) \rightarrow \mathrm{SO}(2,2 n)$. The corresponding embedding $\mathcal{H}_{\mathbb{C}}^{n} \rightarrow \mathcal{X}_{2,2 n}$ is holomorphic but it is not tight. Since $\mathcal{H}_{\mathbb{C}}^{n}$ is of rank one, the totally geodesic embedding extends continuously to a map of the boundary of $\mathcal{H}_{\mathbb{C}}^{n}$, but its image does not lie in the Shilov boundary of $\mathcal{X}_{2,2 n}$.

ExAmple 8.12. In $\operatorname{SL}(4, \mathbb{R})$ there are two copies of $\operatorname{Sp}(4, \mathbb{R})$

$$
\begin{aligned}
& \operatorname{Sp}(4, \mathbb{R})_{A}:=\left\{g \in \mathrm{SL}(4, \mathbb{R}) \mid g^{*} J g=J\right\}, \\
& \operatorname{Sp}(4, \mathbb{R})_{B}:=\left\{g \in \operatorname{SL}(4, \mathbb{R}) \mid g^{*} \tilde{J} g=\tilde{J}\right\},
\end{aligned}
$$

where $J=\left(\begin{array}{cc}0 & \text { Id } \\ - \text { Id } & 0\end{array}\right)$ and $\tilde{J}=\left(\begin{array}{cc}0 & \Lambda \\ -\Lambda & 0\end{array}\right)$ with $\Lambda=\left(\begin{array}{ll}0 & 1 \\ 1 & 0\end{array}\right)$, The two embeddings $i_{A, B}$ : $\mathrm{SL}(2, \mathbb{R}) \rightarrow \mathrm{SL}(4, \mathbb{R})$

$$
\begin{gathered}
i_{A}\left(\left(\begin{array}{ll}
a & b \\
c & d
\end{array}\right)\right)=\left(\begin{array}{ll}
a \mathrm{Id} & b \mathrm{Id} \\
c \mathrm{Id} & d \mathrm{Id}
\end{array}\right) \\
i_{B}\left(\left(\begin{array}{ll}
a & b \\
c & d
\end{array}\right)\right)=\left(\begin{array}{cc}
a \mathrm{Id} & b \Lambda \\
c \Lambda & d \mathrm{Id}
\end{array}\right) .
\end{gathered}
$$

are also conjugate by $s$. The images of $\mathrm{SL}(2, \mathbb{R})$ under these two embeddings are contained in $\operatorname{Sp}(4, \mathbb{R})_{A} \cap \operatorname{Sp}(4, \mathbb{R})_{B}$. The embedding $i_{A}$ is tight and positive with respect to $\operatorname{Sp}(4, \mathbb{R})_{A}$ but totally real with respect to $\operatorname{Sp}(4, \mathbb{R})_{B}$.

The boundary $\partial \mathbb{D}$ of $\mathbb{D}$ is mapped under both embeddings into the Shilov boundaries $\check{S}_{A}$ respectively $\check{S}_{B}$. The totally real embedding extends to an embedding of $\mathrm{SL}(2, \mathbb{C})$ whereas the tight embedding extend to an embedding of $\mathrm{SO}(2,2)$ into $\operatorname{Sp}(4, \mathbb{R})$.

\section{Classification of Tight Embeddings of the Poincaré Disk}

In this section we classify all tight embeddings $f: \mathbb{D} \rightarrow \mathcal{X}$, where $\mathcal{X}$ is any Hermitian symmetric space.

Definition 9.1. Let $\mathcal{X}$ be a Hermitian symmetric space of noncompact type. Let $V \subset \mathcal{X}$ be a subset. The Hermitian hull $\mathcal{H}(V)$ of $V$ is the smallest Hermitian symmetric subspace $\mathcal{H}(V) \subset \mathcal{X}$, such that $V \subset \mathcal{H}(V)$.

If $\mathcal{X}_{1}, \mathcal{X}_{2}$ are Hermitian symmetric spaces and $f: \mathcal{X}_{1} \rightarrow \mathcal{X}_{2}$ is a totally geodesic embedding, we denote by $\mathcal{H}(f)=\mathcal{H}\left(f\left(\mathcal{X}_{1}\right)\right)$ the Hermitian hull of $f\left(\mathcal{X}_{1}\right) \subset \mathcal{X}_{2}$.

REMARK 9.2. We make some observations.

(1) Let $\mathcal{X}_{1}$ be irreducible, then $f: \mathcal{X}_{1} \rightarrow \mathcal{X}_{2}$ is (anti)-holomorphic if and only if $\mathcal{H}(f)=f\left(\mathcal{X}_{1}\right)$.

(2) If $f: \mathcal{X}_{1} \rightarrow \mathcal{X}_{2}$ is tight, then $f: \mathcal{X}_{1} \rightarrow \mathcal{H}(f)$ is tight and $\mathcal{H}(f) \rightarrow \mathcal{X}_{2}$ is tight and holomorphic.

(3) If $f: \mathcal{X}_{1} \rightarrow \mathcal{X}_{2}$ is tight, then $\mathcal{H}(f)$ is of tube type if and only if $\mathcal{X}_{1}$ is of tube type (Theorem 5.8). 
Proposition 9.3. Let $\mathcal{X}_{1}, \mathcal{X}_{2}$ be Hermitian symmetric spaces of tube type and $f: \mathcal{X}_{1} \rightarrow \mathcal{X}_{2}$ a tight embedding with corresponding Lie algebra homomorphism $\rho: \mathfrak{g}_{1} \rightarrow \mathfrak{g}_{2}$. Let $\mathcal{H}(f) \subset \mathcal{X}_{2}$ be the Hermitian hull and $\mathfrak{h} \subset \mathfrak{g}_{2}$ the Lie subalgebra corresponding to the subgroup of Hermitian type $G_{\mathcal{H}(f)}$ determined by $\mathcal{H}(f)$.

Then $\mathfrak{h}$ is the subalgebra generated by $\rho\left(\mathfrak{g}_{1}\right)$ and $Z_{2}$, where $Z_{2} \in \mathfrak{k}_{2}$ is the element defining the complex structure on $\mathcal{X}_{2}$.

Proof. The Hermitian symmetric space $\mathcal{H}(f)$ is of tube type and the embedding $\mathcal{H}(f) \rightarrow \mathcal{X}_{2}$ is tight and holomorphic, therefore the corresponding Lie algebra homomorphism is an (H2) homomorphism (Lemma 8.5). In particular, the element $Z_{\mathfrak{h}}$ in the center of the maximal compact subalgebra of $\mathfrak{h}$ defining the complex structure on $\mathcal{H}(f)$ equals $Z_{2}$. Thus $\left\langle\rho\left(\mathfrak{g}_{1}\right), Z_{2}\right\rangle \subset \mathfrak{h}$, and equality follows from the minimality of $\mathcal{H}(f)$.

Proposition 9.3 allows us to define in the above context $\left(\mathcal{X}_{1}\right.$ and $\mathcal{X}_{2}$ of tube type) the Hermitian hull of the Lie algebra homomorphism $\rho: \mathfrak{g}_{1} \rightarrow \mathfrak{g}_{2}$ as

$$
\mathcal{H}(\rho):=\left\langle\rho\left(\mathfrak{g}_{1}\right), Z_{2}\right\rangle .
$$

REMARK 9.4. A similar characterization of the Hermitian hull is not true if $\mathcal{X}$ is not of tube type. Consider for example the canonical embedding of $\mathfrak{s u}(p, p) \rightarrow \mathfrak{s u}(p, q)$. It is holomorphic and tight, but the central element $Z_{p, q}$ of the maximal compact Lie algebra defining the complex structure on the symmetric space associated to $\mathrm{SU}(p, q)$ is not contained in $\mathfrak{s u}(p, p)$ if $p \neq q$.

Lemma 9.5. Let $\mathcal{X}$ be an irreducible Hermitian symmetric space and $f: \mathbb{D} \rightarrow \mathcal{X}$ a tight embedding with corresponding Lie algebra homomorphism $\rho: \mathfrak{s l}(2, \mathbb{R}) \rightarrow \mathfrak{g}$.

If $\mathcal{H}(\rho)=\mathfrak{g}$, then $\mathfrak{g} \cong \mathfrak{s p}(2 n, \mathbb{R})$ and $\rho: \mathfrak{s l}(2, \mathbb{R}) \rightarrow \mathfrak{g}$ is the $2 n$-dimensional irreducible representation.

Proof. Since $\mathbb{D}$ is of tube type, $\mathcal{H}(\rho)=\mathfrak{g}$ implies that necessarily $\mathcal{X}$ is of tube type. Thus, Proposition 9.3 gives that $\mathfrak{g}=\left\langle\rho(\mathfrak{s l}(2, \mathbb{R})), Z_{\mathcal{J}_{\mathcal{X}}}\right\rangle_{\mathbb{R}}$. Let $\mathfrak{g}_{\mathbb{C}}$ be the complexification of $\mathfrak{g}$ and $\rho_{\mathbb{C}}: \mathfrak{s l}(2, \mathbb{C}) \rightarrow \mathfrak{g}_{\mathbb{C}}$ the complexification of $\rho$, then $\mathfrak{g}_{\mathbb{C}}=$ $\left\langle\rho_{\mathbb{C}}(\mathfrak{s l}(2, \mathbb{C})), Z_{\mathcal{J}_{\mathcal{X}}}\right\rangle_{\mathbb{C}}$

By tightness $Z_{\mathcal{J}_{\mathcal{X}}}$ cannot lie in the centralizer of $\rho(\mathfrak{s l}(2, \mathbb{R}))$ in $\mathfrak{g}$ and so $Z_{\mathcal{J}}$ cannot lie in the centralizer of $\rho_{\mathbb{C}}(\mathfrak{s l}(2, \mathbb{C}))$ in $\mathfrak{g}_{\mathbb{C}}$. Hence, the centralizer $\mathcal{Z}_{\mathfrak{g}_{\mathbb{C}}}\left(\rho_{\mathbb{C}}(\mathfrak{s l}(2, \mathbb{C}))\right)$ is trivial.

This means that $\rho_{\mathbb{C}}(\mathfrak{s l}(2, \mathbb{C}))$ is a semiprincipal three-dimensional simple subalgebra of $\mathfrak{g}_{\mathbb{C}}$. Semiprincipal subalgebras were classified by Dynkin, and we refer the reader to $[\mathrm{Dy}],[\mathrm{V}]$ for more details. Using the classification by Dynkin (see $[\mathrm{Dy}],[\mathrm{V}]$ ), we consider all possible cases of semiprincipal three-dimensional simple subalgebras in $\mathfrak{g}_{\mathbb{C}}$ which are complexifications of three-dimensional simple subalgebras of the specific real form $\mathfrak{g}$ of $\mathfrak{g}_{\mathbb{C}}$. This case by case study gives the following result:

1. When $\mathfrak{g}=\mathfrak{s p}(2 n, \mathbb{R}), \mathfrak{g}_{\mathbb{C}}=\mathfrak{s p}(2 n, \mathbb{C})$ the semiprincipal subalgebra $\mathfrak{h}$ is given by the image of the irreducible representation of $\mathfrak{s l}(2, \mathbb{C}) \rightarrow \mathfrak{s p}(2 n, \mathbb{C})$, hence $\rho: \mathfrak{s l}(2, \mathbb{R}) \rightarrow \mathfrak{s p}(2 n, \mathbb{R})$ is the irreducible representation.

2. When $\mathfrak{g}=\mathfrak{s u}(n, n), \mathfrak{g}_{\mathbb{C}}=\mathfrak{s l}(2 n, \mathbb{C})$ the semiprincipal subalgebra is also given by the irreducible representation of $\mathfrak{s l}(2, \mathbb{C})$, which in dimension $2 n$ is always 
contained in $\mathfrak{s p}(2 n, \mathbb{C})$. Thus we have $\mathcal{H}(\rho)=\left\langle\rho(\mathfrak{s l}(2, \mathbb{R})), Z_{\mathfrak{g}}\right\rangle=\mathfrak{s p}(2 n, \mathbb{R}) \subset$ $\mathfrak{s u}(n, n)$.

3. When $\mathfrak{g}=\mathfrak{s o}(2,2 n-1), \mathfrak{g}_{\mathbb{C}}=\mathfrak{s o}(2 n+1, \mathbb{C})$ the semiprincipal subalgebra $\mathfrak{h}$ is the image of the irreducible representation of $\mathfrak{s l}(2, \mathbb{C})$. But any real irreducible representation of $\mathfrak{s l}(2, \mathbb{R})$ into $\mathfrak{s o}(2,2 n-1)$ is contained either in $\mathfrak{s o}(2,3) \cong$ $\mathfrak{s p}(4, \mathbb{R})$ or $\mathfrak{s o}(2,1) \cong \mathfrak{s p}(2, \mathbb{R})$.

4. In the remaining cases $\mathfrak{g}=\mathfrak{s o}(2,2 n), \mathfrak{s o}^{*}(2 n)$ or $\mathfrak{e}_{V I I}$ there are no semiprincipal three dimensional subalgebras in $\mathfrak{g}_{\mathbb{C}}$ which are complexifications of a real three dimensional simple subalgebra in $\mathfrak{g}$.

Summarizing we get the result: $\mathfrak{g} \cong \mathfrak{s p}(2 n, \mathbb{R})$ and $\rho: \mathfrak{s l}(2, \mathbb{R}) \rightarrow \mathfrak{g}$ is given by the irreducible representation of $\mathfrak{s l}(2, \mathbb{R})$.

Corollary 9.6. Let $f: \mathbb{D} \rightarrow \mathcal{X}$ be a positive tight embedding with corresponding Lie algebra homomorphism $\rho: \mathfrak{s l}(2, \mathbb{R}) \rightarrow \mathfrak{g}$. Then

$$
\mathcal{H}(\rho)=\oplus_{i=1}^{k} \mathfrak{s p}\left(2 n_{i}, \mathbb{R}\right) \subset \mathfrak{g}
$$

with $\sum_{i=1}^{k} n_{i} \leq \mathrm{r}_{\mathcal{X}}$ and $\rho_{i}: \mathfrak{s l}(2, \mathbb{R}) \rightarrow \mathfrak{s p}\left(2 n_{i}, \mathbb{R}\right)$ being the irreducible representation, and

$$
\mathcal{H}(f)=\mathcal{Y}_{1} \times \cdots \times \mathcal{Y}_{k},
$$

where $\mathcal{Y}_{k}$ is a symmetric space associated to $\operatorname{Sp}\left(2 n_{i}, \mathbb{R}\right)$.

Proof. The subalgebra $\mathcal{H}(\rho)$ is a semisimple Lie algebra of Hermitian type, so $\mathcal{H}(\rho)=\oplus_{i=1}^{k} \mathfrak{h}_{i}$, where all simple factors $\mathfrak{h}_{i}$ are of tube type. The representations $\rho_{i}: \mathfrak{s l}(2, \mathbb{R}) \rightarrow \mathfrak{h}_{i}$ correspond again to tight embeddings with $\mathfrak{h}_{i}=\mathcal{H}\left(\rho_{i}\right)$. Hence Lemma 9.5 implies the claim.

\section{A Appendix}

A.1 The norm of the bounded Kähler class. We give here a proof of the following:

Theorem A.1. Let $M$ be a connected simple Lie group with finite center and assume that its associated symmetric space $\mathcal{M}$ is Hermitian. Let $\kappa_{M}^{\mathrm{b}} \in \mathrm{H}_{\mathrm{cb}}^{2}(M, \mathbb{R})$ be the continuous bounded class given by the Kähler form associated to the Hermitian metric of holomorphic sectional curvature -1 . Then

$$
\left\|\kappa_{M}^{\mathrm{b}}\right\|=\frac{\mathrm{r}_{\mathcal{M}}}{2} .
$$

Since $\kappa_{\mathcal{M}}^{\mathrm{b}}$ is defined by the cocycle $c_{\omega_{\mathcal{M}}}$ which, according to Theorem 2.8 is bounded by $r_{\mathcal{M}} / 2$, the inequality

$$
\left\|\kappa_{M}^{\mathrm{b}}\right\| \leq \frac{\mathrm{r}_{\mathcal{M}}}{2}
$$

follows. Observe that the opposite inequality cannot be immediately deduced from the statement that

$$
\left\|c_{\omega_{\mathcal{M}}}\right\|_{\infty}=\frac{\mathrm{r}_{\mathcal{M}}}{2}
$$


since the norm $\left\|\kappa_{\mathcal{M}}^{\mathrm{b}}\right\|$ is the infimum of the supremum norms over all bounded cocycles on $\mathcal{M}$ representing $\kappa_{\mathcal{M}}^{\mathrm{b}}$.

We shall proceed as follows: let $d: \mathbb{D} \rightarrow \mathcal{M}$ be a diagonal disk (see Definition 2.18) and $\rho: L \rightarrow M$ the corresponding homomorphism, where $L$ is some finite covering of $\mathrm{SU}(1,1)$. Then

$$
d^{*}\left(\omega_{\mathcal{M}}\right)=\mathrm{r}_{\mathcal{M}} \omega_{\mathbb{D}}
$$

and hence it follows from Lemma 2.15 that

$$
\rho_{\mathrm{b}}^{*}\left(\kappa_{M}^{\mathrm{b}}\right)=\mathrm{r}_{\mathcal{M}} \kappa_{L}^{\mathrm{b}} .
$$

Since the pullback in continuous bounded cohomology is norm decreasing, we have

$$
\left\|\kappa_{M}^{\mathrm{b}}\right\| \geq\left\|\rho_{\mathrm{b}}^{*}\left(\kappa_{M}^{\mathrm{b}}\right)\right\|=\mathrm{r}_{\mathcal{M}}\left\|\kappa_{L}^{\mathrm{b}}\right\|
$$

and it suffices to determine the values of $\left\|\kappa_{L}^{\mathrm{b}}\right\|$. Thus the theorem will follow from the following:

Proposition A.2. With the above notation we have that

$$
\left\|\kappa_{L}^{\mathrm{b}}\right\|=\frac{1}{2} \text {. }
$$

Proof. Let $e:(\partial \mathbb{D})^{3} \rightarrow\{-1,0,1\}$ be the orientation cocycle on the circle $\partial \mathbb{D}$. we use the fact that the space of $L$-invariant alternating bounded measurable cocycles on $\partial \mathbb{D}$ is isometrically isomorphic to $\mathrm{H}_{\mathrm{cb}}^{2}(L, \mathbb{R})$ and that, under this isomorphism, $e$ corresponds to $2 \kappa_{L}^{\mathrm{b}}$, [Io]. Thus, since $\|e\|_{\infty}=1$, we deduce that $\left\|\kappa_{L}^{\mathrm{b}}\right\|=1 / 2$.

\section{A.2 Surjection onto lattices.}

Proposition A.3. Let $\Gamma$ be a countable discrete group, $G$ a Lie group of Hermitian type and $\rho: \Gamma \rightarrow G$ a homomorphism such that the image $\rho(\Gamma)$ is Zariski dense and the action of $\Gamma$ on the Shilov boundary of the associated symmetric space is minimal. Then $\rho$ is tight.

The proof of this proposition relies on functoriality properties of bounded cohomology. We use that the bounded continuous cohomology $\mathrm{H}_{\mathrm{b}}^{2}(L, \mathbb{R})$ in degree two of a locally compact group $L$ can be realized isometrically as the space $\mathcal{Z} L_{\text {alt }}^{\infty}\left(B^{3}, \mathbb{R}\right)^{L}$ of $L$-invariant bounded alternating $L^{\infty}$ cocycles on any space $(B, \nu)$ on which the $L$-action is amenable and mixing. In particular if $G$ is a group of Hermitian type, then

$$
\mathrm{H}_{\mathrm{b}}^{2}(G, \mathbb{R}) \cong \mathcal{Z} L_{\mathrm{alt}}^{\infty}\left((G / P)^{3}, \mathbb{R}\right)^{G},
$$

where $P<G$ is a minimal parabolic subgroup. Likewise, we use that if $\Gamma$ is the countable discrete group with a probability measure $\theta$ then a Poisson boundary $(B, \nu)$ for $(\Gamma, \theta)$ always exists and then

$$
\mathrm{H}_{\mathrm{b}}^{2}(\Gamma, \mathbb{R}) \cong \mathcal{Z} L_{\text {alt }}^{\infty}\left(B^{3}, \mathbb{R}\right)^{\Gamma} .
$$

For more details and proofs of the precise functoriality properties we refer the reader to $[$ BuIoW3, §4] and to the references therein.

Proof. We realize the Shilov boundary of the bounded domain realization $\mathcal{D}$ of the symmetric space associated to $G$ as $\check{S}=G / Q$. We fix a minimal parabolic subgroup $P<Q<G$ and denote by pr: $G / P \rightarrow G / Q$ the canonical projection. If 
$\beta_{\mathcal{D}}: \check{S}^{3} \rightarrow \mathbb{R}$ is the normalized Bergmann cocycle, then $\operatorname{pr}^{*} \beta_{\mathcal{D}}:(G / P)^{3} \rightarrow \mathbb{R}$ is a cocycle in $L_{\mathrm{alt}}^{\infty}\left((G / P)^{3}, \mathbb{R}\right)^{G}$ representing the class $\kappa_{G}^{\mathrm{b}} \in \mathrm{H}_{\mathrm{cb}}^{2}(G, \mathbb{R})$. It follows from (A.1) that

$$
\left\|\kappa_{G}^{\mathrm{b}}\right\|=\operatorname{ess}_{x_{1}, x_{2}, x_{3} \in G / P} \operatorname{pr}^{*} \beta_{\mathcal{D}}\left(x_{1}, x_{2}, x_{3}\right) .
$$

Since the image of $\rho$ is Zariski dense, there exists a $\rho$-equivariant measurable boundary map $\varphi:(B, \nu) \rightarrow G / P$ [BuIoW3, Th. 4.7], and moreover $\varphi^{*} \operatorname{pr}^{*} \beta_{\mathcal{D}}$ represents $\rho_{\mathrm{b}}^{*} \kappa_{G}^{\mathrm{b}}$ [BuIoW3, Prop. 4.6].

The essential image of $\operatorname{pr} \circ \varphi:(B, \nu) \rightarrow G / Q$ is defined as the support of the push-forward measure $(\operatorname{pr} \circ \varphi)^{*}(\nu)$ and is hence a closed $\rho(\Gamma)$-invariant subset which, by minimality of the $\Gamma$-action, must be equal to $G / Q$. But then this implies that

$$
\operatorname{ess~sup}_{x_{1}, x_{2}, x_{3} \in G / P}\left|\operatorname{pr}^{*} \beta_{\mathcal{D}}\left(x_{1}, x_{2}, x_{3}\right)\right|=\underset{b_{1}, b_{2}, b_{3} \in G / P}{\operatorname{ess} \sup }\left|\operatorname{pr}^{*} \beta_{\mathcal{D}}\left(\varphi\left(b_{1}\right), \varphi\left(b_{2}\right), \varphi\left(b_{3}\right)\right)\right|,
$$

and hence

$$
\left\|\kappa_{G}^{\mathrm{b}}\right\|=\left\|\rho_{\mathrm{b}}^{*} \kappa_{G}^{\mathrm{b}}\right\|
$$

From the above proposition we immediately obtain the following:

Corollary A.4. Let $\Gamma$ be a countable discrete group, $G$ a Lie group of Hermitian type and $\rho: \Gamma \rightarrow G$ a homomorphism. If $\rho(\Gamma)$ contains a lattice $\Lambda<G$, then $\rho$ is tight.

Corollary A.5. Let Mod $_{g}$ be the mapping class group of a closed oriented surface of genus $g$. Then the natural homomorphism $\rho: \operatorname{Mod}_{g} \rightarrow \operatorname{Sp}(2 g, \mathbb{R})$ is tight. In particular if $\kappa_{G}^{\mathrm{b}} \in \mathrm{H}_{\mathrm{cb}}^{2}(\mathrm{Sp}(2 g, \mathbb{R}))$ is the bounded Kähler class associated to the normalized Kähler form, then the norm of $\rho_{\mathrm{b}}^{*} \kappa_{G}^{\mathrm{b}} \in \mathrm{H}_{\mathrm{b}}^{2}\left(\mathbf{M o d}_{g}, \mathbb{R}\right)$ is $g / 2$.

Proof. The natural homomorphism $\rho: \operatorname{Mod}_{g} \rightarrow \operatorname{Sp}(2 g, \mathbb{R})$ surjects onto $\operatorname{Sp}(2 g, \mathbb{Z})$, so $\left\|\rho_{\mathrm{b}}^{*} \kappa_{G}^{\mathrm{b}}\right\|=\left\|\kappa_{G}^{\mathrm{b}}\right\|$ which equals $g / 2$ by Theorem A.1.

\section{References}

[BGSS] E. Breulllard, T. Gelander, J. Souto, P. Storm, Dense embeddings of surface groups, Geom. Topol. 10 (2006), 1373-1389 (electronic).

[BuIo1] M. Burger, A. IozzI, Bounded Kähler class rigidity of actions on Hermitian symmetric spaces, Ann. Sci. École Norm. Sup. (4) 37:1 (2004), 77-103.

[BuIo2] M. Burger, A. IozzI, Bounded differential forms, generalized Milnor-Wood inequality and an application to deformation rigidity, Pure Appl. Math. Q. 125:1 (2007), 1-23.

[BuIoLW] M. Burger, A. Iozzi, F. Labourie, A. Wienhard, Maximal representations of surface groups: Symplectic Anosov structures, Special Issue: In Memory of Armand Borel, Part 2 of 3. Quarterly Journal of Pure and Applied Mathematics $1: 3$ (2005), 555-601.

[BuIoW1] M. Burger, A. Iozzi, A. Wienhard, Surface group representations with maximal Toledo invariant, Annals of Math., to appear; http: //www.arXiv.org/math.DG/0605656.

[BuIoW2] M. Burger, A. Iozzi, A. Wienhard, Surface group representations with maximal Toledo invariant, C. R. Acad. Sci. Paris, Sér. I 336 (2003), 387-390. 
[BuIoW3] M. Burger, A. Iozzi, A. Wienhard, Hermitian symmetric spaces and Kähler rigidity, Transform. Groups 12:1 (2007), 5-32.

[BuM1] M. Burger, N. Monod, Bounded cohomology of lattices in higher rank Lie groups, J. Eur. Math. Soc. 1:2 (1999), 199-235.

[BuM2] M. Burger, N. Monod, Continuous bounded cohomology and applications to rigidity theory, Geom. Funct. Anal. 12 (2002), 219-280.

[CØ] J.L. Clerc, B. Ørsted, The Gromov norm of the Kaehler class and the Maslov index, Asian J. Math. 7:2 (2003), 269-295.

[DT] A. Domic, D. Toledo, The Gromov norm of the Kaehler class of symmetric domains, Math. Ann. 276:3 (1987), 425-432.

[Dy] E.B. Dynkin, Semisimple subalgebras of semisimple Lie algebras, Am. Math. Soc., Transl. Ser. II, AMS 6 (1957), 111-243.

[E] W.T. van Est, Group cohomology and Lie algebra cohomology in Lie groups, I, II, Nederl. Akad. Wetensch. Proc. Series A 56=Indag. Math. 15 (1953), 484-504.

[I1] S.-I. IHARA, Holomorphic imbeddings of symmetric domains into a symmetric domain, Proc. Japan Acad. 42 (1966), 193-197.

[I2] S.-I. IhARA, Holomorphic imbeddings of symmetric domains, J. Math. Soc. Japan 19 (1967), 261-302.

[Io] A. IozzI, Bounded cohomology, boundary maps, and representations into Homeo $_{+}\left(S^{1}\right)$ and $S U(1, n)$, in "Rigidity in Dynamics and Geometry, Cambridge, UK, 2000", Springer Verlag (2002), 237-260.

[KW] A. Korányi, J.A. Wolf, Realization of Hermitian symmetric spaces as generalized half-planes, Ann. of Math. (2) 81 (1965), 265-288.

$[\mathrm{KoM}] \quad$ V. Koziarz, J. Maubon, Representations of complex hyperbolic lattices into rank 2 classical Lie groups of Hermitian type, preprint; http://www .arXiv.org/math/0703174.

[L] O. Loos, Bounded symmetric domains and Jordan pairs, Department of Mathematics, University of California at Irvine, Mathematical Lectures, 1977.

[M] N. Monod, Continuous Bounded Cohomology of Locally Compact Groups, Springer Lecture Notes in Math. 1758 (2001).

[S1] I. SATAKE, On representations and compactifications of symmetric Riemannian spaces, Ann. of Math. (2) 71 (1960), 77-110.

[S2] I. Satake, Algebraic Structures of Symmetric Domains, Kanô Memorial Lectures 4, Iwanami Shoten, Tokyo, 1980.

[V] È.B. Vinberg (ED.), Lie groups and Lie algebras, III, Structure of Lie groups and Lie algebras, Encyclopaedia of Mathematical Sciences 41, Springer-Verlag, Berlin (1994); Current Problems in Mathematics. Fundamental Directions 41 (in Russian), Akad. Nauk SSSR, Vsesoyuz. Inst. Nauchn. i Tekhn. Inform., Moscow (1990).

Marc Burger, FIM, ETH Zentrum, Rämistrasse 101, CH-8092 Zürich, Switzerland burger@math.ethz.ch

Alessandra IozzI, Department Mathematik, ETH Zentrum, Rämistrasse 101, CH-8092 Zürich, Switzerland iozzi@math.ethz.ch Anna Wienhard, Department of Mathematics, Princeton University, Fine Hall, Washington Road, Princeton, NJ 08540, USA

wienhard@math.princeton.edu

Received: February 2, 2008

Revision: July 22, 2008

Accepted: July 29, 2008 